\title{
Diversity Combining of Signals With Different Modulation Levels and Constellation RearRangement in CoOperative Relay Networks
}

$$
\text { by }
$$

\section{Akram Salem Bin Sediq}

B. Sc. in Electrical Engineering, American University of Sharjah, Sharjah, United Arab Emirates, 2006

\author{
A THESIS SUBMITTED TO THE \\ Faculty of Graduate Studies and Research \\ IN PARTIAL FULFILLMENT OF THE REQUIREMENTS FOR THE DEGREE OF \\ Master of Applied Science in Electrical Engineering
}

Ottawa-Carleton Institute for Electrical and Computer Engineering

Department of Systems and Computer Engineering

Carleton University

Ottawa, Ontario

September 2008

(C) Akram Salem Bin Sediq, 2008 


$\begin{array}{ll}\begin{array}{l}\text { Library and } \\ \text { Archives Canada }\end{array} & \begin{array}{l}\text { Bibliothèque et } \\ \text { Archives Canada }\end{array} \\ \begin{array}{l}\text { Published Heritage } \\ \text { Branch }\end{array} & \begin{array}{l}\text { Direction du } \\ \text { Patrimoine de l'édition }\end{array} \\ \begin{array}{l}\text { 395 Wellington Street } \\ \text { Ottawa ON K1A ON4 } \\ \text { Canada }\end{array} & \begin{array}{l}\text { 395, rue Wellington } \\ \text { Ottawa ON K1A 0N4 } \\ \text { Canada }\end{array}\end{array}$

Your file Votre référence ISBN: 978-0-494-44030-8

Our file Notre référence

ISBN: 978-0-494-44030-8

NOTICE:

The author has granted a nonexclusive license allowing Library and Archives Canada to reproduce, publish, archive, preserve, conserve, communicate to the public by telecommunication or on the Internet, loan, distribute and sell theses worldwide, for commercial or noncommercial purposes, in microform, paper, electronic and/or any other formats.

The author retains copyright ownership and moral rights in this thesis. Neither the thesis nor substantial extracts from it may be printed or otherwise reproduced without the author's permission.
AVIS:

L'auteur a accordé une licence non exclusive permettant à la Bibliothèque et Archives Canada de reproduire, publier, archiver, sauvegarder, conserver, transmettre au public par télécommunication ou par l'Internet, prêter, distribuer et vendre des thèses partout dans le monde, à des fins commerciales ou autres, sur support microforme, papier, électronique et/ou autres formats.

L'auteur conserve la propriété du droit d'auteur et des droits moraux qui protège cette thèse. $\mathrm{Ni}$ la thèse ni des extraits substantiels de celle-ci ne doivent être imprimés ou autrement reproduits sans son autorisation.
In compliance with the Canadian

Privacy Act some supporting forms may have been removed from this thesis.

While these forms may be included in the document page count, their removal does not represent any loss of content from the thesis.
Conformément à la loi canadienne sur la protection de la vie privée, quelques formulaires secondaires ont été enlevés de cette thèse.

Bien que ces formulaires aient inclus dans la pagination, il n'y aura aucun contenu manquant.

\section{Canada}




\section{Abstract}

In this thesis, two problems in cooperative relay networks are tackled, namely, diversity combining of signals with different modulation levels, and Constellation Rearrangement (CoRe).

In digital cooperative relaying, signals from the source-destination and relaydestination links are combined at the destination to achieve spatial diversity. The vast majority of the research done in cooperative relaying assumes the modulation level used by both the source and relay to be the same. This assumption does not necessarily hold in next generation wireless networks where adaptive modulation is implemented and in such a case, conventional maximal ratio combining does not work . This raises the need to investigate different receiver structures and understands the performance gain as well as the complexity associated with each receiver. Consequently, performance analysis as well as simulation results of the BER of different receiver structures are presented. We show that performing soft-bit maximal ratio combining is the most attractive receiver structure, as it yields near optimal BER performance with low complexity. In the case of nomadic relay networks, where error propagation is a limiting factor in the BER performance, link adaptive regeneration (LAR) can be effectively used. Moreover, we propose a modified LAR scheme that significantly outperforms its conventional counterpart, without any increase in the complexity. The gain of the proposed scheme is illustrated through both analysis and simulation.

The problem of CoRe is defined as finding good bit to symbol mapping for each transmitting node, without changing the modulation level. Through exhaustive numerical search, we propose a good CoRe scheme. Unlike most of the existing CoRe schemes, the proposed CoRe scheme does not use Gray-coding constellation in any of the transmitting nodes. In the context of fixed relays, the proposed CoRe scheme achieves significant gain compared to the conventional scheme and it outperforms the existing CoRe techniques. In the context of nomadic relays, we observe that the proposed CoRe, compared to conventional and other existing CoRe schemes, is the most sensitive scheme to error propagation. More importantly, in order for any CoRe scheme to have better performance than the conventional scheme, the average SNR in the source-relay link must be greater than a threshold value that is a function of both the average SNRs in the source-destination and relay-destination links. Otherwise, the CoRe schemes degrade the BER performance as they amplify the undesirable effect of error propagation. In nomadic relay networks, the proposed CoRe outperforms the conventional and the existing CoRe schemes if and only if the average SNR in the source-relay link is greater than this threshold. 


\section{Acknowledgements}

First and foremost, I am grateful to Allah (God) for all that I am and all that I have. I am very thankful for the patient guidance and the endless support and encouragement given by my supervisor Dr. Halim Yanikomeroglu. I am also thankful to the defence committee members, Dr. Ian Marsland, Dr. Yongyi Mao, and Dr. Ashraf Matrawy, for their valuable comments and discussions which greatly improved the content and the presentation of this thesis.

I would like to express my gratitude to Muhammad Al-Juaid and Ziad El-Khatib for their continuous support since my first day in Ottawa. Their assistance can never be appreciated enough. I would like also to express my gratefulness to Feroz Bokhari for his valuable comments and suggestions regarding this work. I deeply appreciate his help in proofreading my thesis and my manuscripts before submissions. Many thanks go to my colleagues, Ahmed Abdelsalam, Dr. Abdulkareem Adinoyi, Dr. Basak Can, Fruzan Atay, Furkan Alaca, Ghassan Dahman, Houda Chafnaji, Jason Lee, Jung-Min Park, Mahmudur Rahman, Matthew Dorrance, Mohamed Rashad, Saad Al-Ahmadi, Sebastian Szyszkowicz, Soumitra Dixit, Tarik Shehata, and Dr. Youssouf Mouhamedou for their encouragement.

The work was supported in part by an Ontario Graduate Scholarship (OGS) for international students. I thankfully acknowledge this support.

To my parents, brothers, and sisters, I must express my sincere appreciation for their guidance, support, and love in every aspect of my life. Finally, I thank my lovely wife Areej for her patience, encouragement, support, and love. 


\section{Table of Contents}

Abstract $\quad$ ii

Acknowledgements

Table of Contents $\quad$ iv

List of Tables $\quad$ vi

List of Figures $\quad$ vii

List of Acronyms ix

List of Symbols $\quad$ xi

Chapter 1: Introduction 1

1.1 Cooperative Relay Networks . . . . . . . . . . . . . . . . . 2

1.2 Thesis Motivation and Objectives . . . . . . . . . . . . . 4

1.2.1 Diversity combining of signals with different modulation levels 4

1.2.2 Constellation Rearrangement for Cooperative Relay Networks 5

1.3 Thesis Contributions . . . . . . . . . . . . . . . . 6

1.4 Published, Submitted, and Proposed Manuscripts . . . . . . . . . . 8

1.5 Thesis Organization . . . . . . . . . . . . . . . . . 9

$\begin{array}{lll}\text { Chapter 2: } & \text { System Model } & 11\end{array}$

Chapter 3: Conventional Selection Combining (SC) and BER Selection Combining (BSC) 15

3.1 Performance Analysis of SC . . . . . . . . . . . . . . . . 16

3.2 Performance Analysis of BSC . . . . . . . . . . . . . . . . . . 19

3.3 Comparison between $\mathrm{SC}$ and $\mathrm{BSC} \ldots \ldots \ldots . \ldots . \ldots 21$

Chapter 4: Optimal and Near-Optimal Diversity Combining of Signals with Different Modulation Levels 25

4.1 The Optimal Detector: The Maximum Likelihood Detector(MLD) . . 26

4.1.1 Receiver structure . . . . . . . . . . . . . . 26, 26

4.1.2 Square M-QAM modulation with Gray-Coding . . . . . . . . . 26

4.1.3 Advantages and disadvantages of the MLD . . . . . . . . . . . 31

4.2 Soft-Bit Maximum Likelihood Detector (SBMLD) . . . . . . . . . 33 
4.3 Soft-Bit Maximal Ratio Combiner (SBMRC) . . . . . . . . . . 41

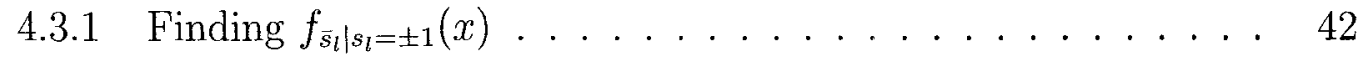

4.3 .2 Finding the instantaneous $B E R \ldots \ldots \ldots \ldots \ldots 4$

4.3 .3 Finding the average $B E R \ldots \ldots \ldots \ldots \ldots$

4.4 Analytical and Simulation Results . . . . . . . . . . . . . 49

4.5 Adaptive Modulation and Diversity Combining . . . . . . . . . 54

4.5.1 Dynamic adaptive modulation . . . . . . . . . . 54

4.5.2 Static adaptive modulation . . . . . . . . . . . 57

Chapter 5: Diversity Combining of Signals with Different Modulation Levels in Nomadic Relay Networks $\quad 61$

5.1 Existing techniques to mitigate error propagations . . . . . . . . 61

5.2 Link Adaptive Regeneration . . . . . . . . . . . . . . . . . 64

5.3 Modified Link Adaptive Regeneration . . . . . . . . . . . . . . . . . 65

5.4 Performance Analysis of LAR and modified LAR . . . . . . . . 66

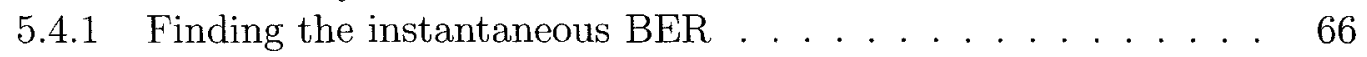

5.4 .2 Finding the average BER . . . . . . . . . . . . 68

5.5 Analytical and Simulation Results . . . . . . . . . . . . . . 69

Chapter 6: Constellation Rearrangement (CoRe) in Cooperative Relay Networks

6.1 Review of Hybrid Automatic Repeat reQuest and Constellation Rearrangement . . . . . . . . . . . . . . . . 76

6.2 System Model for CoRe in Cooperative Relaying . . . . . . . . . . . 79

6.3 Proposed CoRe for Cooperative Relaying . . . . . . . . . . 80

6.4 Augmented Signal Constellation . . . . . . . . . . . . . . . 84

6.5 Simulation Results . . . . . . . . . . . . . . . . . . . 90

$\begin{array}{lll}\text { Chapter 7: } & \text { Conclusions and Future Work } & 96\end{array}$

7.1 Summary and Contributions . . . . . . . . . . . . . 96

7.2 Future Work . . . . . . . . . . . . . . . . . . . . . . 100

$\begin{array}{ll}\text { References } & 102\end{array}$

Appendix A: Confidence Interval Analysis 106

Appendix B: Different Gray Coded 16-QAM Constellations 108

Appendix C: Derivation of $I_{0}$ and $I_{1} \quad 110$

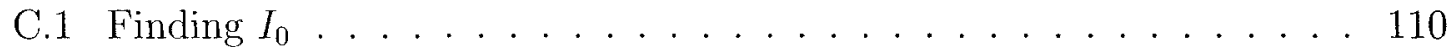

C.2 Finding $I_{1} \ldots \ldots \ldots \ldots \ldots \ldots \ldots \ldots \ldots \ldots \ldots$

Appendix D: Proof that it is Always Better to Assign $M_{0} \leq M_{1} \quad 113$ 


\section{List of Tables}

4.1 The matrices $\boldsymbol{\lambda}^{+}$and $\boldsymbol{\lambda}^{-}$for different M-QAM constellations. . . . . 38 4.2 Loss in SNR (dB) at BER $=10^{-3}$ of SC, BSC, SBMLD and SBMRC compared to the optimum MLD. . . . . . . . . . . . 52

4.3 The modes for the dynamic mode of operation. . . . . . . . . . 56

4.4 The modes for the static mode of operation. . . . . . . . . . . 59 


\section{List of Figures}

1.1 Digital Cooperative Relaying. . . . . . . . . . . . . . . 4

2.1 System Model . . . . . . . . . . . . . . . . . . . . . . 11

2.2 Frame Structure . . . . . . . . . . . . . . . . . . . . . 12

3.1 BER performance of SC. . . . . . . . . . . . . . . . . . 19

3.2 BER performance of BSC. . . . . . . . . . . . . . . . . 22

3.3 BER performance of SC and BSC . . . . . . . . . . . . 24

4.1 The MLD block diagram. . . . . . . . . . . . . . . . . . 27

4.24 -QAM (QPSK) constellation with Gray-coding . . . . . . . . . 28

4.3 16-QAM constellation with Gray-coding. . . . . . . . . . . . . 32

4.4 64-QAM constellation with Gray-coding. . . . . . . . . . . . . . 32

4.5 The SBMLD block diagram. . . . . . . . . . . . . . . . . 35

4.6 Conditional PDF of $\tilde{s}_{i, 0}\left(\tilde{s}_{i, 2}\right)$ generated from a 16-QAM symbol given

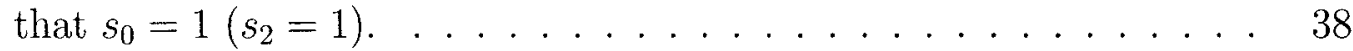

4.7 Conditional PDF of $\tilde{s}_{i, 1}\left(\tilde{s}_{i, 3}\right)$ generated from a 16-QAM symbol given that $s_{1}=1\left(s_{3}=1\right) \ldots \ldots \ldots \ldots$

4.8 Conditional PDF of $\tilde{s}_{i, 0}\left(\tilde{s}_{i, 3}\right)$ generated from a $64-\mathrm{QAM}$ symbol given that $s_{0}=1\left(s_{3}=1\right) \ldots \ldots \ldots . \ldots \ldots$

4.9 Conditional PDF of $\tilde{s}_{i, 1}\left(\tilde{s}_{i, 4}\right)$ generated from a $64-\mathrm{QAM}$ symbol given that $s_{1}=1\left(s_{4}=1\right) \ldots \ldots \ldots 40$

4.10 Conditional PDF of $\tilde{s}_{i, 2}\left(\tilde{s}_{i, 5}\right)$ generated from a 64-QAM symbol given that $s_{2}=1\left(s_{5}=1\right) \ldots \ldots \ldots \ldots$. . . . . . . . . 40

4.11 The SBMRC block diagram . . . . . . . . . . . . . . . . . . 42

4.12 Instantaneous BER performance of SBMRC for $\left(M_{0}=4, M_{1}=16\right) . \quad . \quad 47$

4.13 Instantaneous BER performance of SBMRC for $\left(M_{0}=4, M_{1}=64\right)$. . 47

4.14 Instantaneous BER performance of SBMRC for $\left(M_{0}=16, M_{1}=64\right) . \quad 48$

4.15 BER performance of diversity combining using SBMRC for $L=2$. . 52

4.16 BER performance of diversity combining using SBMRC for $L=3$. . 53

4.17 BER performance of SC, BSC, and SBMRC . . . . . . . . . . . 53

4.18 The SNR regions for each mode in the dynamic adaptive modulation. $\quad 57$

4.19 The SNR regions for each mode in the static adaptive modulation. . . 60

5.1 BER performance of combining QPSK and 16-QAM using SBMRC for different strategies. 
5.2 BER performance of combining QPSK and 64-QAM using SBMRC for different strategies. . . . . . . . . . . . . . . . 72

5.3 BER performance of combining 16QAM and 64-QAM using SBMRC for different strategies. . . . . . . . . . . . . . . 73

5.4 BER performance of combining QPSK, 16-QAM and 16-QAM using SBMRC for different strategies. . . . . . . . . . . . . . 73

5.5 BER performance of LAR using both simulation and analytical lower bound. . . . . . . . . . . . . . . . . . . . 74

5.6 BER performance of modified LAR using both simulation and analytical lower bound. . . . . . . . . . . . . . . . . . . 74

5.7 BER performance of both LAR and modified LAR as a function of

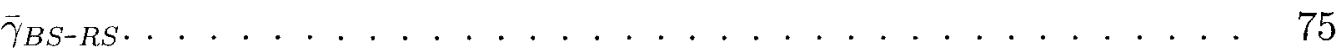

6.1 Bit labeling of the real part of 16-QAM, for CoRe $1 \ldots \ldots \ldots 78$

6.2 Bit labeling of the real part of 64-QAM, for CoRe 1. . . . . . . 78

6.3 Bit labeling of the real part of 16-QAM, for CoRe $2 \ldots \ldots \ldots \ldots .79$

6.4 Bit labeling of the real part of 64-QAM, for CoRe 2. . . . . . . . 79

6.5 Bit labeling of the real part of 16-QAM, for the proposed CoRe. . . . 83

6.6 Bit labeling of the real part of 64-QAM, for the proposed CoRe. . . . 83

6.7 The augmented signal constellation of 4-PAM for conventional scheme. 86

6.8 The augmented signal constellation of 8-PAM for conventional scheme. 86

6.9 The augmented signal constellation of 4-PAM for CoRe $1 . \ldots . .887$

6.10 The augmented signal constellation of 8-PAM for CoRe 1. . . . . 87

6.11 The augmented signal constellation of 4-PAM for CoRe $2 . \ldots . . .88$

6.12 The augmented signal constellation of 8-PAM for CoRe 2. . . . . 88

6.13 The augmented signal constellation of 4-PAM for the proposed CoRe. 89

6.14 The augmented signal constellation of 8-PAM for the proposed CoRe. 89

6.15 BER of different CoRe schemes in a fixed relay network, $M=16 . \quad$. . 91

6.16 BER of different CoRe schemes in a fixed relay network, $M=64 . \quad$. . 91

6.17 BER of different CoRe schemes in a nomadic relay network, $M=16 . \quad 93$

6.18 BER of different CoRe schemes in a nomadic relay network, $M=64 . \quad 93$

6.19 BER of different CoRe schemes as a function of $\bar{\gamma}_{B S-R S}, M=16 \ldots \quad$. . 95

6.20 BER of different CoRe schemes as a function of $\bar{\gamma}_{B S-R S}, M=64 \ldots .95$

A.1 The normalized confidence interval for different confidence levels. . . 107

B.1 Different Gray coded 16-QAM constellations. . . . . . . . . . . . 109 


\section{List of Acronyms}

$\begin{array}{ll}\text { 4G } & \text { Fourth Generation } \\ \text { AF } & \text { Amplify and Forward } \\ \text { AG } & \text { Asymptotic Gain } \\ \text { AMC } & \text { Adaptive Modulation and Coding } \\ \text { ARQ } & \text { Automatic Repeat reQuest } \\ \text { AWGN } & \text { Additive White Gaussian Noise } \\ \text { BER } & \text { Bit Error Ratio } \\ \text { BS } & \text { Base Station } \\ \text { BSC } & \text { BER Selection Combining } \\ \text { CDMA } & \text { Code Division Multiple Access } \\ \text { C-MRC } & \text { Cooperative MRC } \\ \text { CoRe } & \text { Constellation Rearrangement } \\ \text { CRC } & \text { Cyclic Redundancy Check } \\ \text { CSI } & \text { Channel State Information } \\ \text { dB } & \text { Decibel } \\ \text { DF } & \text { Decode and Forward } \\ \text { DQPSK } & \text { Differential Quadrature Phase Shift Keying } \\ \text { FEC } & \text { Forward Error Control coding } \\ \text { HARQ } & \text { Hybrid Automatic Repeat reQuest } \\ \text { LAR } & \text { Link Regenerative Relaying } \\ \text { LLR } & \text { Log-Likelihood Ratio } \\ \text { LCM } & \text { Least Common Multiple } \\ \text { MIMO } & \text { Multiple Input Multiple Output } \\ \text { MLD } & \text { Maximum Likelihood Detector } \\ \text { MRC } & \text { Maximal Ratio Combiner } \\ \text { OFDM } & \text { Orthogonal Frequency Divison Multiplexing } \\ \text { PAM } & \text { Pulse Amplitude Modulation } \\ \text { PDF } & \text { Probability Density Function } \\ \text { QAM } & \text { Quadrature Amplitude Modulation } \\ \text { QPSK } & \text { Quadrature Phase Shift Keying } \\ \text { RF } & \text { Radio Frequency } \\ \text { RS } & \text { Relay Station } \\ \text { SED } & \text { squared Euclidean distance } \\ \text { SBMLD } & \text { Soft-Bit Maximum Likelihood Detector } \\ \text { SBMRC } & \text { Soft-Bit Maximal Ratio Combiner } \\ \text { SC } & \text { Conventional Selection Combining } \\ \text { SNR } & \text { Signal to Noise Raio } \\ \text { TDM } & \text { Time Division Multiplexing } \\ & \\ & \end{array}$


TDMA Time Division Multiple Access

UT User Terminal 


\section{List of Symbols}

\begin{tabular}{|c|c|}
\hline $\begin{array}{l}L \\
\alpha_{i} \\
\bar{\gamma}_{B S-R S_{i}} \\
\bar{\gamma}_{B S-U T_{i}} \\
\bar{\gamma}_{R S-U T_{i}} \\
\bar{\gamma}_{i} \\
\bar{s}_{l}\end{array}$ & $\begin{array}{l}\text { Number of transmitting nodes } \\
\text { channel coefficient between transmitting node } i \text { and user terminal } \\
\text { average SNR of the BS-RS } S_{i} \text { link } \\
\text { average SNR of the BS-UT } i \text { link } \\
\text { average SNR of the RS-UT } T_{i} \text { link } \\
\text { average SNR of link from transmitting node } i \text { to UT } \\
l^{\text {th }} \text { wieghted sum of soft-bits }\end{array}$ \\
\hline $\mathbf{S}$ & A modulated symbol in augmented signal constellation \\
\hline$\gamma_{B S-R S_{i}}$ & instantaneous SNR of the BS-RS $S_{i}$ link \\
\hline$\gamma_{B S-U T_{i}}$ & instantaneous SNR of the BS-UT $T_{i}$ link \\
\hline$\gamma_{R S-U T_{i}}$ & instantaneous SNR of the $\mathrm{RS}^{-U_{T}}$ link \\
\hline$\gamma_{i}$ & instantaneous SNR of link from transmitting node $i$ to UT \\
\hline $\begin{array}{l}\gamma_{\text {out }} \\
\hat{s}_{l}\end{array}$ & $\begin{array}{l}\text { Post processing SNR at the output of the combiner } \\
l^{\text {th }} \text { decoded bit }\end{array}$ \\
\hline$\kappa_{i}$ & power scaling coeffeceint used at $\mathrm{RS}_{i}$ in LAR \\
\hline & augmented signal constellation \\
\hline$\sigma_{i}^{2}$ & $E\left\{\left|\alpha_{i}^{2}\right|\right\}$ \\
\hline$\tilde{s}_{i, l}$ & $l^{t h}$ soft-bit in sub-frame $i$ \\
\hline$B E R_{\text {inst }}$ & Instantaneous BER \\
\hline$C$ & Least Common Multiple of $\left\{K_{0}, K_{1}, \ldots, K_{L-1}\right\}$ \\
\hline$D^{B S C}$ & SNR gain achieved by BSC \\
\hline$D^{S C}$ & SNR gain achieved by SC \\
\hline$d_{M_{i}}$ & $\begin{array}{l}\text { A constant used to make the average energy per bit for the } M_{i} \text {-QAM } \\
\text { constellation equal to unity }\end{array}$ \\
\hline$f_{\tilde{s}_{l} \mid s_{l}= \pm 1}(x)$ & Conditional PDF of $\bar{s}_{l}$ given $s_{l}= \pm 1$ \\
\hline$f_{\tilde{s}_{i, k_{i}} \mid s_{k_{i}}}= \pm 1(x)$ & Conditional PDF of $\tilde{s}_{i, k_{i}}$ given $s_{k_{i}}= \pm 1$ \\
\hline & Index of transmitting node \\
\hline$K_{i}$ & number of bits per $M_{i}$-QAM symbol \\
\hline$M_{i}$ & modulation level used by transmitting node $i$ \\
\hline $\begin{array}{l}n_{i, j} \\
N_{0}\end{array}$ & $\begin{array}{l}\text { complex AWGN in received symbol in } i^{\text {th }} \text { sub-frame and } j^{\text {th }} \text { time-slot } \\
\text { Noise spectral density }\end{array}$ \\
\hline
\end{tabular}


$P_{t} \quad$ Targeted BER

$r_{i, j}^{M_{i}} \quad$ received symbol in $i^{\text {th }}$ sub-frame and $j^{\text {th }}$ time-slot

$R S_{i} \quad i^{\text {th }}$ relay

$S_{i, j}^{M_{i}} \quad M_{i}$-QAM symbol transmitted by node $i$ in $j^{\text {th }}$ time slot

$s_{l} \quad l^{\text {th }}$ original information bit

$T_{i} \quad$ number of $M_{i}$-QAM symbols transmitted by node $i$ in $i^{\text {th }}$ sub-frame 


\section{Chapter 1}

\section{Introduction}

The major upcoming milestone in wireless cellular networks is the development and standardization of the fourth generation $(4 \mathrm{G})$ cellular networks . The $4 \mathrm{G}$ networks promise to provide the end user with cost-effective ubiquitous high data rates of up to $100 \mathrm{Mb} / \mathrm{s}$ for mobile users and up to $1 \mathrm{~Gb} / \mathrm{s}$ for stationary users. These ambitious goals are not feasible using the conventional cellular architecture for the following reasons. Firstly, as the required data rate increases, the transmitting power must increases linearly with the same proportion, in order to maintain the same energy per bit, and thus the same Bit Error Ratio (BER). This is a hefty price to pay since the complexity of Radio Frequency $(\mathrm{RF})$ circuits increases dramatically as the transmitting power increases. Moreover, there is a limit for the maximum transmitted power in order to avoid health hazards associated with high transmitted power. Secondly, the envisioned spectrum for the $4 \mathrm{G}$ will be above the 2-Ghz band. In this band, the radio signals are more susceptible to non-line of sight conditions. Although increasing the number of base stations in the network will overcome the aforementioned problems, it is economically not feasible. [1]

Recently, the concept of infrastructure-based multihop networks was proposed as promising network architecture to achieve the $4 \mathrm{G}$-envisioned high data rates. Unlike the current network structure where the communication happens between the base 
station (BS) and the user terminal (UT), multihop communications propose to have fixed or nomadic relay stations (RS) to relay the signals between UT and the BS. In other words, the signal may travel from the source to destination through multiple hops. Among the many features of relay networks, providing coverage to users that experience high path loss (heavy shadowing) is the main attractive feature, as most of the signal processing techniques fail to function in such conditions. [1]

\subsection{Cooperative Relay Networks}

Relays can be classified into digital and analog relays. Analog relays amplifyand-forward $(\mathrm{AF})$ the received signal without any decoding while digital relays fully decode and forward (DF) a regenerated version of the received signal. In this thesis, digital relaying is considered as it is the focus of most of the next generation wireless networks standards such as IEEE $802.16 \mathrm{j} / \mathrm{m}$ [2] and LTE-Advanced [3]. Relays can be further classified into fixed and nomadic relays. As the name implies, fixed relays are deployed by the service provider in strategic locations while nomadic relays are mobile relays that can be provided by the service provider or can be idle UTs that help other UTs. In this thesis, we address both fixed and nomadic relays. Since fixed relays are installed at strategic locations, Line-of-Sight transmission between the BS and the RS can be achieved in most cases. Consequently, from the physical layer prospective, the difference between fixed and nomadic RSs is that, in the former, the link from BS to RS is reliable and can be assumed error free, for all practical purposes. However, the error free assumption does not hold in the case of nomadic RSs. In nomadic relays, the errors made at the RS propagate to the destination. This undesirable phenomena is called error propagation and it is a limiting factor for the BER performance. 
Due to the limitation of the signal-processing hardware, the RS can't transmit and receive in the same channel $[1,4]$. Thus, the RS will operate in half duplex mode and orthogonality must be maintained between transmission and reception, which requires an extra channel to be allocated for relaying purposes. Although orthogonality can be attained through time, frequency, and/or Code-Division Multiple Access (CDMA), for simplicity, we assume throughout the thesis that orthogonality is maintained through Time-Division Multiple Access (TDMA).

Instead of relying solely on the signal from the last hop, cooperative relaying proposes to utilize all the signals in the intermediate hops and do diversity combining to combat fading $[4,5]$. For example, consider the single-user single-relay network depicted in Fig. 1.1. In the first time slot, the BS transmits the signal to the RS and the signal is overheard by the UT, because of the broadcast nature of the electromagnetic waves. The RS fully decodes the signal and forwards it to the UT in the next time slot. In conventional relaying, the UT decodes the signal from the RS only. In cooperative relaying, the UT properly performs diversity combining of the signals it receives from the BS and RS. Since these signals experience uncorrelated fading, combining achieves diversity and thus reduces the effect of fading significantly. It is worth mentioning that diversity is achieved in this case without the need for installing multiple antennas, either at the transmitter, or at the receiver. This feature makes cooperative diversity more appealing for small mobile terminals, than transmit or receive diversity that relies on multiple-input multiple-output (MIMO), such as those proposed in [6-8]. However, the diversity gain achieved by cooperative relaying comes at the price of using extra radio resources for relays. Nevertheless, it is shown in [9] that the gain from cooperation offsets the incurred loss in radio resources. 


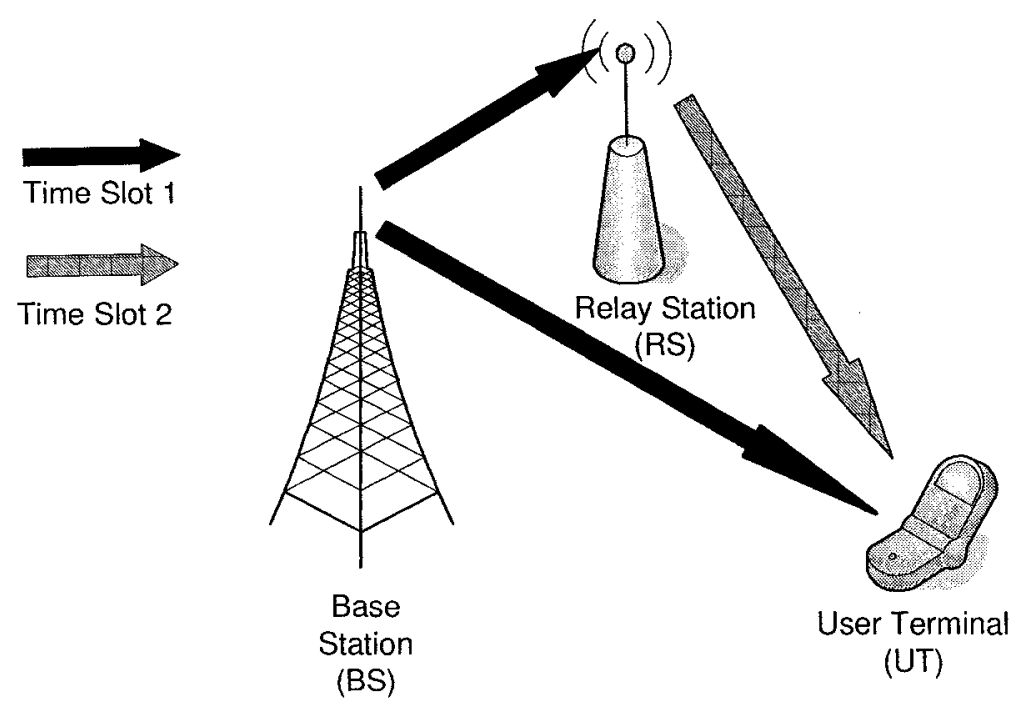

Figure 1.1: Digital Cooperative Relaying.

\subsection{Thesis Motivation and Objectives}

\subsubsection{Diversity combining of signals with different modulation levels}

Two key strategies proposed for increasing the data rate in relay networks are cooperative relaying $[4,5]$, and adaptive modulation and coding (AMC). While the former is used to improve the quality of the links by combining the signals received from the BS and the RSs, the latter is used to optimize the transmission rate according to the channel conditions. In [10] and [11], it is shown that the average throughput of the wireless network can be significantly increased by combining the two strategies. When AMC is utilized, the signals reaching the UT from BS and RS do not necessarily belong to the same modulation, yet they contain the same information bits. In order to achieve spatial diversity for signals with different modulation levels, selection combining, rather than maximal ratio combining (MRC), has been proposed to be utilized because it has not been known yet how to do MRC for signals with different modulation levels $[10,11]$. Beside selection combining, a trivial solution is to force the 
modulation levels to be the same in all the links, and optimally combine at UT using MRC. However, this solution reduces the benefit of AMC as the performance will be limited by the link which suffers from the most unfavourable channel conditions. In [12], a receiver structure for satellite-based digital audio transmission is proposed to process signals that contain the same information but transmitted using different multiplexing schemes (such as TDM and OFDM). Note that the term modulation is used in [12] as a synonym of multiplexing. The modulation schemes employed were QPSK and DQPSK and MRC was used to optimally combine the signals. To the best of our knowledge, the optimal technique of combining signals with different modulation schemes has not investigated yet, and this is one of the main objectives of the thesis.

The need for such a technique arises from the nature of relay networks that impose different channel conditions on different links. For example, for fixed relay networks, since the relays are fixed and can be installed at strategic locations, reliable line-ofsight $\operatorname{link}(\mathrm{s})$ can be established between BS and RS, and hence, larger constellations can be used to achieve high data rates. However, because of the mobility of the users, the $\operatorname{link}(\mathrm{s})$ from RS to UT are not necessary as reliable, so smaller constellations can be used to ensure reliable transmission. In order to achieve spatial diversity at $\mathrm{UT}$, it is imperative to establish an optimal diversity combining scheme for different modulation levels. We restrict our work to square M-QAM modulations as they are the most popular schemes in wireless networks [13].

\subsubsection{Constellation Rearrangement for Cooperative Relay Networks}

Hybrid Automatic Repeat reQuest (HARQ) is an error control mechanism that constitutes an essential part in data communications. This mechanism enables error 
free transmission of data packet through multiple transmission of the same packet until it is decoded successfully at the receiver. The receiver can be designed to store the erroneously decoded packets and combine them to improve the BER performance. In $[14-17]$, it is shown that varying the bit labeling in the constellation for each transmission without changing the modulation level can further improve the BER performance after combining. This is called Constellation Rearrangement (CoRe). In [18], it is realized that the performance of HARQ is similar to cooperative relaying in fixed relay environment, although in the latter the retransmission happens at the relay. Therefore, in this work the same CoRe that is proposed in [14] was applied to cooperative relaying.

Motivated by the significant gain achieved by using the CoRe concept, and the similarities between HARQ and cooperative relaying, we intend to develop a new CoRe scheme that outperforms the other existing CoRe schemes proposed in [14-17]. In all the previous works, it is assumed that Gray-coding is used for at least the first transmission. We expect better performance by relaxing this assumption. Moreover, we answer the question, whether cooperation benefits from CoRe in nomadic relay networks or not, noting that the conclusions made by [18] were restricted to cooperation in fixed relay networks.

\subsection{Thesis Contributions}

The key contributions of this thesis are the following:

- Proposing BER selection combining (BSC) which significantly outperforms conventional selection combining (SC) when the signals to be combined happen to be from different modulation levels. This performance gain comes at no penalty 
in complexity.

- Developing closed-form BER expressions for both SC and BSC when they are used for combining signals with different modulation levels. Moreover, the asymptotic gain obtained by using BSC is analytically quantified.

- Deriving the optimal maximum likelihood detector (MLD) for combining signals with different modulation levels.

- Studying the performance of two suboptimal receiver structures which operate on bit-by-bit basis that we refer to as soft-bit maximal ratio combining (SBMRC) and soft-bit maximum likelihood detector (SBMLD). SBMRC is in essence identical to LLR combining ${ }^{1}$ used in HARQ ${ }^{2}$.

- Developing a very tight BER bound for SBMRC when it is used for combining signals with different modulation levels. Since the performance of SBMRC is very close to that of both MLD and SBMLD, the developed bound can be also used to approximate the BER performance of both MLD and SBMLD.

- Comparing the BER performance results of different diversity combining schemes for different scenarios using both simulation and analytical results.

- Illustrating that Link Adaptive Regeneration (LAR) performs very well to mitigate error propagation when the signals to be combined belong to different modulation levels.

\footnotetext{
${ }^{1}$ Sometimes also referred to as soft-combining or Chase combining.

${ }^{2}$ The norm in HARQ is to use the same modulation level in all the retransmissions, as far as we know.
} 
- Introducing the modified LAR, which outperforms LAR when different modulation levels are used, without any increase in the complexity.

- Proposing a CoRe scheme that outperforms the existing CoRe schemes. The proposed CoRe does not use Gary-coding in any of the transmitting nodes.

- Highlighting the effect of error propagation in the BER performance of different CoRe schemes, including the proposed CoRe.

\subsection{Published, Submitted, and Proposed Manuscripts}

\section{Chapter 3}

- Akram Bin Sediq and Halim Yanikomeroglu, "Performance analysis of selection combining and BER selection combining in combining signals with different modulation levels", submitted to IEEE Transaction on Wireless Communications, September 2008.

\section{Chapter 4}

- Akram Bin Sediq and Halim Yanikomeroglu, "Diversity combining of signals with different modulation levels in cooperative relay networks", presented in the Wireless World Research Forum Meeting 20 (WWRF20), April 2008, Ottawa, Canada.

- Akram Bin Sediq and Halim Yanikomeroglu, "Diversity combining of signals with different modulation levels in cooperative relay networks", accepted for IEEE VTC2008 Fall, September 2008, Calgary, Alberta, Canada. 
- Akram Bin Sediq and Halim Yanikomeroglu, "Performance analysis of soft-bit maximal ratio combining in cooperative relay networks", submitted to IEEE Transaction on Wireless Communications, September 2008.

\section{Chapter 5}

- Akram Bin Sediq and Halim Yanikomeroglu, "Performance analysis of link adaptive regeneration in cooperative relay networks", to be submitted to IEEE Transaction on Wireless Communications.

\section{Chapter 6}

- Akram Bin Sediq and Halim Yanikomeroglu, "An Improved constellation rearrangement scheme for cooperative relay networks", to be submitted to IEEE Transaction on Wireless Communications.

\subsection{Thesis Organization}

The remainder of this thesis is organized as follows. In Chapter 2 we describe the system model for cooperative relay network. In Chapter 3, we review SC and propose BSC. Pefromance analysis of the BER of both SC and BSC is also presented in the same chapter. In Chapter 4, we investigate MLD, SBMLD, and SBMRC for diversity combining of signals with different modulations levels in fixed relay networks. The performance analysis as well as the simulation results of BER performance are also presented. In Chapter 5, we explain how to use SBMRC in nomadic relay networks without significant suffering from the error propagation phenomena. We start by a literature survey of the existing techniques proposed to mitigate error propagation under the assumption that the BS and RS use the same modulation level. Among 
the different techniques, we implement the LAR strategy proposed in [19]. Then, we propose the modified LAR and through simulation of the BER performance, we show significant gain associated with the use of the modified LAR as compared to the conventional LAR. Unlike Chapters 3, 4, and 5, where we deal with combining signals of different modulation levels, in Chapter 6, we study the case when the same modulation level is used by the transmitting nodes but with different bit labeling in the constellation which is called CoRe. We start by reviewing the existing CoRe techniques that were proposed originally for HARQ. Then we highlight the similarities and differences between cooperative relay networks and HARQ, and exploit these differences to design a new CoRe scheme that outperforms all existing CoRe schemes.

Finally, conclusions and the major contributions of this thesis are outlined in Chapter 7. Moreover, we highlight a number of research areas for future work. 


\section{Chapter 2}

\section{System Model}

We consider a multihop network of $L$ transmitting nodes (comprising of $L-1$ RSs and a BS), and a receiving UT, all having a single antenna. This layout is shown in Fig. 2.1. The RSs are used to assist a UT which suffers from poor channel conditions. The RSs fully decode the signals they receive from the BS and forward them to the $\mathrm{UT}$.

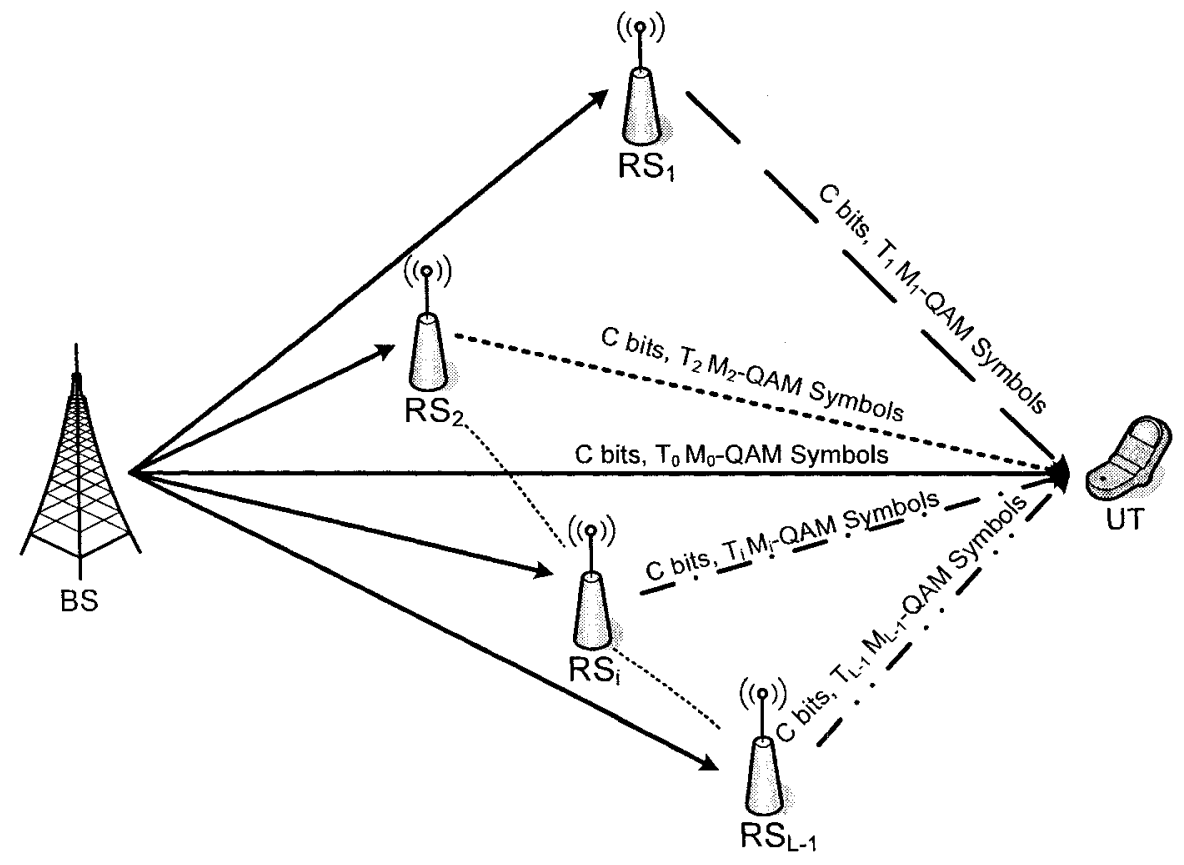

Figure 2.1: System Model 
The transmitting nodes transmit on $L$ orthogonal channels, i.e., they do not interfere with each other. For simplicity, we consider TDMA to insure orthogonal transmission from all the nodes. The transmitting node $i,\left(\mathrm{BS}\right.$ or $\left.\mathrm{RS}_{i}\right)$ where $i \in$ $\{0,1, \ldots, L-1\}$, uses square $M_{i}$-Quadrature Amplitude Modulation $\left(M_{i^{-}}\right.$QAM) with Gray coding. Each $M_{i}$-QAM symbol carries $K_{i}$ bits, where $K_{i}=\log _{2}\left(M_{i}\right)$ and $M_{i}$ is the $i^{\text {th }}$ modulation level. Without loss of generality, the $M_{i}$-QAM constellation has an average energy per bit equal to unity. We focus on these modulation schemes as they are the most popular schemes in wireless networks [13].

The frame is divided into $L$ sub-frames, i.e., one sub-frame for each transmitting node. All sub-frames contain the same sequence of bits, denoted by $\left\{s_{0}, s_{1}, \ldots, s_{C-1}\right\}$. The $i^{\text {th }}$ sub-frame consists of $T_{i} M_{i}$-QAM symbols, each denoted by $S_{i, j}^{M_{i}}$, where $j \in$ $\left\{0,1, \ldots, T_{i}-1\right\}$. The symbol $S_{i, j}^{M_{i}}$ contains the bit sequence $\left\{s_{j K_{i}+0}, s_{j K_{i}+1}, \ldots, s_{(j+1) K_{i}-1}\right\}$. Note that different nodes will be assigned different number of symbols, depending on their modulation schemes, i.e., $T_{i}=C / K_{i}$. Since $T_{i}$ is an integer, $C$ must be a common multiple of $\left\{K_{0}, K_{1}, \ldots, K_{L-1}\right\}$. Without loss of generality, $C$ will be used as the Least Common Multiple (LCM) of $\left\{K_{0}, K_{1}, \ldots, K_{L-1}\right\}$.

In the zeroth sub-frame, BS broadcasts $T_{0} M_{0^{-}} \mathrm{QAM}$ symbols to all RSs and the UT. Since the RSs can be installed at strategic locations, LOS transmission between BS and the RSs can be achieved. Therefore, the RSs can decode the signals with negligible errors [10]. In the $i^{\text {th }}$ sub-frame, $\mathrm{RS}_{i}$ forwards $T_{i}$ symbols to UT using $M_{i}$-QAM modulation. The frame structure is depicted in Fig. 2.2 .

\begin{tabular}{|c|c|c|c|}
\hline$T_{0}$ symbols $=C$ bits & $T_{1}$ symbols $=C$ bits & $T_{i}$ symbols $=C$ bits & $T_{L-1}$ symbols $=C$ bits \\
\hline$S_{0,0}^{M_{0}}, S_{0,1}^{M_{0}}, \ldots, S_{0, T_{0}-1}^{M_{0}}$ & $S_{1,0}^{M_{1}}, S_{1,1}^{M_{1}}, \ldots, S_{1, T_{1}-1}^{M_{1}}$ & $S_{i, 0}^{M_{i}}, S_{i, 1}^{M_{i}}, \ldots, S_{i, T_{i}-1}^{\overline{M_{i}}}$ & $S_{L-1,0}^{M_{L-1}}, S_{L-1,1}^{M_{L-1}}$ \\
\hline Sub-frame "0" & Sub-frame "1" & Sub-frame " $i$ " & Sub-frame " $L-1$ " \\
\hline $\mathrm{BS} \rightarrow\left\{\mathrm{UT}, \mathrm{RS}_{i}\right\}_{i=1}^{L-1}$ & $\mathrm{RS}_{1} \rightarrow \mathrm{UT}$ & $\mathrm{RS}_{i} \rightarrow \mathrm{UT}$ & $\mathrm{RS}_{L-1} \rightarrow \mathrm{UT}$ \\
\hline
\end{tabular}

Figure 2.2: Frame Structure 
In the $i^{t h}$ sub-frame and the $j^{t h}$ symbol, the received signal at UT is $r_{i, j}^{M_{i}}$ and given by $r_{i, j}^{M_{i}}=\alpha_{i} S_{i, j}^{M_{i}}+n_{i, j}$. The complex additive white Gaussian noise (AWGN) is represented by $n_{i, j}$, and it is modeled as circular symmetric complex Gaussian random variable with zero mean and variance $N_{0}\left(C N\left(0, N_{0}\right)\right)$. The channel coefficient between the transmitting node $i$ and UT is denoted by $\alpha_{i}$ and it captures both large scale fading (path loss and shadowing) and small scale fading due to multipath propagation. Slow fading is assumed, i.e., channel does not change for the whole subframe. It is assumed that $\alpha_{i}$ 's are known at the receiver and modeled as independent $C N\left(0, \sigma_{i}^{2}\right)$, with $\sigma_{i}^{2}=E\left\{\left|\alpha_{i}^{2}\right|\right\}$ (Rayleigh fading). If full channel state information (CSI) is available at the BS, optimizing the modulation levels for all the transmitting nodes can improve the end to end throughput drastically. Such optimization is studied extensively in [10] and [11] and will be revisited in Section 4.5. The instantaneous SNR per bit of the link from BS to UT is $\gamma_{B S-U T}=\left|\alpha_{0}\right|^{2}$ SNR and the average SNR is $\bar{\gamma}_{B S-U T}=\sigma_{0}^{2} \mathbf{S N R}$, where SNR is a reference signal to noise ratio and it is equal to $\mathrm{SNR}=E_{b} / N_{0}$. The instantaneous signal to noise ratio (SNR) per bit of the link from $\mathrm{RS}_{i}$ to UT is $\gamma_{R S_{i}-U T}=\left|\alpha_{i}\right|^{2} \mathbf{S N R}$ and the average SNR is $\bar{\gamma}_{R S_{i}-U T}=\sigma_{i}^{2} \mathbf{S N R}$. In the context of nomadic relays, the channels from BS to RSs are also assumed to be Rayleigh fading and the following notations are used:

$\gamma_{B S-R S_{i}}, \bar{\gamma}_{B S-R S_{i}}$ : instantaneous and average SNR of the link BS-RS ${ }_{i}$, respectively $\gamma_{B S-U T}, \bar{\gamma}_{B S-U T}$ : instantaneous and average SNR of the link BS-UT, respectively $\gamma_{R S_{i}-U T}, \bar{\gamma}_{R S_{i}-U T}$ : instantaneous and average SNR of the link $\mathrm{RS}_{i}-\mathrm{UT}$, respectively.

In the context of fixed relay networks where the SNRs of the links from BS to RSs are not needed as these links are assumed to be error-free, we simplify the notation of the SNRs as follows: $\gamma_{0}=\gamma_{B S-U T}, \bar{\gamma}_{0}=\bar{\gamma}_{B S-U T}, \gamma_{i}=\gamma_{R S_{2}-U T}, \bar{\gamma}_{i}=\bar{\gamma}_{R S_{i}-U T}$.

After receiving all the sub-frames, UT can utilize the signals received from $L$ 
independent branches, and achieve spatial diversity.

We remark that the most general case of $L-1$ relays is considered for mathematical completeness. However, given that each relay requires an orthogonal channel, it will be difficult in practice to have more than two relays due to the limited radio resources.

To accurately simulate the BER, the simulator is implemented to estimate the actual BER with an inaccuracy of utmost $\pm 12 \%$ of the actual value, with $95 \%$ confidence level. The inaccuracy decreases for large BER, e.g., the inaccuracy for a BER of $10^{-3}$ is utmost $\pm 6 \%$. These inaccuracies are acceptable for all practical purposes [20]. Moreover, in most of the cases, analytical BER expressions are derived to support the simulation results. (See appendix A for the detailed confidence interval calculation.) 


\section{Chapter 3}

\section{Conventional Selection Combining (SC) and BER Selection Combining (BSC)}

In [11], diversity combining of signals with different modulation levels has been dealt with as follow. First, signals with the same modulations are combined using MRC. Then, the signals from the MRC combiners are decoded one-by-one until a packet is decoded correctly, with the help of a cyclic redundancy check (CRC) scheme. For example, for the case where $M_{0}=16, M_{1}=16$, and $M_{2}=4, \mathrm{MRC}$ is first used to combine the signals from branch 0 and 1 . Then, the combined signal is decoded and checked using CRC. If the the signal is decoded correctly, no more processing is needed. If not, the signal from branch 2 is decoded.

In [10], the BS has full CSI of all the links, and selection diversity is achieved by transmitting only through the link that achieves the highest throughput.

Since in this work we do not assume CSI at the BS and we do not employ CRC, we will use conventional selection combining (SC) and BER selection combining (BSC). In $\mathrm{SC}$, the receiver decodes the signal only from the branch that has the maximum SNR. When different modulations are employed, the branch that has the maximum SNR may not necessarily be the most reliable link due to different error-resistance capabilities of the different modulations. Consequently, we introduce BSC as a better selection combining scheme in which the receiver decodes the signal from the branch 
that has the minimum BER.

Using the approximate BER expression for square M-QAM given in [21], the selection criterion can be written as

$$
\text { Select branch } i \text {, where } i=\arg \min _{i} B E R_{M_{i}} \text {. }
$$

In the above

$$
B E R_{M_{i}}=c_{M_{i}} Q\left(\sqrt{2 d_{M_{i}}^{2} \gamma_{i}}\right)
$$

where

$$
d_{M_{i}}=\sqrt{\frac{3 \log _{2} M_{i}}{2\left(M_{i}-1\right)}} \text {, and } c_{M_{i}}=\frac{2\left(1-1 / M_{i}\right)}{\log _{2} M_{i}} .
$$

Note that $\mathrm{BSC}$ reduces to $\mathrm{SC}$ in the special case where all the signals belong to the same modulation. Although the literature is rich in the BER performance analysis of SC [22], it is limited to the case of combining similar modulation schemes. Consequently, in the subsequent sections we present the performance analysis of both $\mathrm{SC}$ and the proposed BSC in combining signals with different modulations levels. For mathematical tractability, we limit the analysis to the case of single RS $(L=2)$. We remark that even though our focus in this thesis is on square M-QAM modulations, the derived equations in this chapter are applicable to any modulation scheme that has instantaneous BER in the form $c_{M_{i}} Q\left(\sqrt{2 d_{M_{i}}^{2} \gamma}\right)$.

\subsection{Performance Analysis of SC}

The instantaneous BER at the output of SC, given $\gamma_{0}$ and $\gamma_{1}$, can be written as

$$
B E R_{\text {inst }}=\left\{\begin{array}{l}
c_{M_{0}} Q\left(\sqrt{2 d_{M_{0}}^{2} \gamma_{0}}\right), \gamma_{0} \geq \gamma_{1} \\
c_{M_{1}} Q\left(\sqrt{2 d_{M_{1}}^{2} \gamma_{1}}\right), \gamma_{0}<\gamma_{1}
\end{array}\right.
$$

The common approach in deriving the average BER is to average the instantaneous BER over the PDF of the output SNR [22]. However, this approach does not work in 
our problem since the instantaneous BER is a piecewise function with intervals that are dependant on the instantaneous SNRs. Recall that the PDF of the output SNR does not carry information regarding the instantaneous SNRs. Consequently, to get the average BER, we average (3.4) over the joint PDF of $\gamma_{0}$ and $\gamma_{1}$. Since the channel coefficients are modeled as independent Rayleigh fading random variables, $\gamma_{0}$ and $\gamma_{1}$ are modeled as independent exponential random variables [22,23]. As a result, the joint PDF is the multiplication of the individual PDFs and can be expressed as

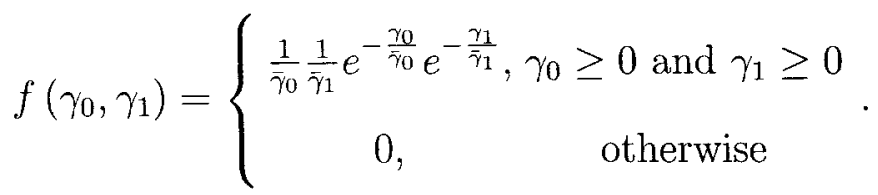

Using (3.4) and (3.5), the average BER can be written as

$$
\begin{aligned}
B E R & =\int_{0}^{\infty} \int_{0}^{\gamma_{1}} c_{M_{1}} Q\left(\sqrt{2 d_{M_{1}}^{2} \gamma_{1}}\right) \frac{1}{\bar{\gamma}_{0}} \frac{1}{\bar{\gamma}_{1}} e^{-\frac{\gamma_{0}}{\bar{\gamma}_{0}}} e^{-\frac{\gamma_{1}}{\bar{\gamma}_{1}}} d \gamma_{0} d \gamma_{1} \\
& +\int_{0}^{\infty} \int_{\gamma_{1}}^{\infty} c_{M_{0}} Q\left(\sqrt{2 d_{M_{0}}^{2} \gamma_{0}}\right) \frac{1}{\bar{\gamma}_{0}} \frac{1}{\bar{\gamma}_{1}} e^{-\frac{\gamma_{0}}{\bar{\gamma}_{0}}} e^{-\frac{\gamma_{1}}{\bar{\gamma}_{1}}} d \gamma_{0} d \gamma_{1} .
\end{aligned}
$$

To simplify the above expression, we define the following function:

$$
\begin{aligned}
H(x ; a, b, c) & =\int a Q(\sqrt{2 b x}) \frac{1}{c} e^{-\frac{x}{c}} \\
& =-a\left(0.5 \sqrt{\frac{b c}{1+b c}}\left(1-2 Q\left(\sqrt{\frac{(1+b c)}{c} 2 x}\right)\right)+Q(\sqrt{2 b x}) e^{-\frac{x}{c}}\right)
\end{aligned}
$$

where the previous integration is evaluated in [24, Appendix A]. Moreover, we define the following function:

$$
\begin{aligned}
J(x ; a, b, c, d)= & \int H(x ; a, b, c) \frac{1}{d} e^{-\frac{x}{d}} d x \\
= & -\int 0.5 a \sqrt{\frac{b c}{1+b c}} \frac{1}{d} e^{-\frac{x}{d}} d x+2 a \int \sqrt{\frac{b c}{1+b c}} Q\left(\sqrt{\frac{(1+b c)}{c} 2 x}\right) \frac{1}{d} e^{-\frac{x}{d}} d x \\
& -\int a Q(\sqrt{2 b x}) e^{-\frac{x}{c} \frac{1}{d}} e^{-\frac{x}{d}} d x \\
= & \left(0.5 a \sqrt{\frac{b c}{1+b c}} e^{-\frac{x}{d}}+H\left(x ; a \sqrt{\frac{b c}{1+b c}}, \frac{(1+b c)}{c}, d\right)-H\left(x ; a \frac{c}{c+d}, b, \frac{c d}{c+d}\right)\right) .
\end{aligned}
$$


The average BER can be expressed in terms of the function $H(x ; a, b, c, d)$ as

$$
\begin{aligned}
B E R= & \int_{0}^{\infty} c_{M_{1}} Q\left(\sqrt{2 d_{M_{1}}^{2} \gamma_{1}}\right)\left(1-e^{-\frac{\gamma_{1}}{\bar{\gamma}_{0}}}\right) \frac{1}{\bar{\gamma}_{1}} e^{-\frac{\gamma_{1}}{\bar{\gamma}_{1}}} d \gamma_{1} \\
& +\int_{0}^{\infty}\left(H\left(\infty ; c_{M_{0}}, d_{M_{0}}^{2}, \bar{\gamma}_{0}\right)-H\left(\gamma_{1} ; c_{M_{0}}, d_{M_{0}}^{2}, \bar{\gamma}_{0}\right)\right) \frac{1}{\bar{\gamma}_{1}} e^{-\frac{\gamma_{1}}{\bar{\gamma}_{1}}} d \gamma_{1} .
\end{aligned}
$$

By evaluating the previous definite integral, the average BER can be expressed in terms of the functions $H(x ; a, b, c, d)$ and $J(x ; a, b, c, d)$ as

$$
\begin{aligned}
B E R= & H\left(\gamma_{1} ; c_{M_{1}}, d_{M_{1}}^{2}, \bar{\gamma}_{1}\right)-H\left(\gamma_{1} ; c_{M_{1}} \frac{\bar{\gamma}_{0}}{\bar{\gamma}_{0}+\bar{\gamma}_{1}}, d_{M_{1}}^{2}, \frac{\bar{\gamma}_{0} \bar{\gamma}_{1}}{\bar{\gamma}_{0}+\bar{\gamma}_{1}}\right) \\
& \left.-H\left(\infty ; c_{M_{0}}, d_{M_{0}}^{2}, \bar{\gamma}_{0}\right) e^{-\frac{\gamma_{1}}{\bar{\gamma}_{1}}}-J\left(\gamma_{1} ; c_{M_{0}}, d_{M_{0}}^{2}, \bar{\gamma}_{0}, \bar{\gamma}_{1}\right)\right]_{\gamma_{1}=0}^{\gamma_{1}=\infty} \\
= & H\left(\infty ; c_{M_{1}}, d_{M_{1}}^{2}, \bar{\gamma}_{1}\right)-H\left(\infty ; c_{M_{1}} \frac{\bar{\gamma}_{0}}{\bar{\gamma}_{0}+\bar{\gamma}_{1}}, d_{M_{1}}^{2}, \frac{\bar{\gamma}_{0} \bar{\gamma}_{1}}{\bar{\gamma}_{0}+\bar{\gamma}_{1}}\right)+H\left(\infty ; c_{M_{0}}, d_{M_{0}}^{2}, \bar{\gamma}_{0}\right) \\
& -J\left(\infty ; c_{M_{0}}, d_{M_{0}}^{2}, \bar{\gamma}_{0}, \bar{\gamma}_{1}\right)-H\left(0 ; c_{M_{1}}, d_{M_{1}}^{2}, \bar{\gamma}_{1}\right)+H\left(0 ; c_{M_{1}} \frac{\bar{\gamma}_{0}}{\bar{\gamma}_{0}+\bar{\gamma}_{1}}, d_{M_{1}}^{2}, \frac{\bar{\gamma}_{0} \bar{\gamma}_{1}}{\bar{\gamma}_{0}+\bar{\gamma}_{1}}\right) \\
& +J\left(0 ; c_{M_{0}}, d_{M_{0}}^{2}, \bar{\gamma}_{0}, \bar{\gamma}_{1}\right) .
\end{aligned}
$$

Finally, we evaluate (3.10) using (3.7) and (3.8). After considerable simplifications, the average BER can be explicitly written as

$$
\begin{aligned}
B E R= & \frac{1}{2} c_{M_{0}}\left(1-\sqrt{\frac{d_{M_{0}}^{2} \bar{\gamma}_{0}}{1+d_{M_{0}}^{2} \bar{\gamma}_{0}}}\right)+\frac{1}{2} c_{M_{1}}\left(1-\sqrt{\frac{d_{M_{1}}^{2} \bar{\gamma}_{1}}{1+d_{M_{1}}^{2} \bar{\gamma}_{1}}}\right) \\
& -\frac{1}{2} c_{M_{0}} \frac{\bar{\gamma}_{1}}{\bar{\gamma}_{0}+\bar{\gamma}_{1}}\left(1-\sqrt{\frac{d_{M_{0}}^{2} \bar{\gamma}_{2}}{1+d_{M_{0}}^{2} \bar{\gamma}_{2}}}\right)-\frac{1}{2} c_{M_{1}} \frac{\bar{\gamma}_{0}}{\bar{\gamma}_{0}+\bar{\gamma}_{1}}\left(1-\sqrt{\frac{d_{M_{1}}^{2} \bar{\gamma}_{2}}{1+d_{M_{1}}^{2} \bar{\gamma}_{2}}}\right),
\end{aligned}
$$

where $\bar{\gamma}_{2} \triangleq \frac{\bar{\gamma}_{0} \bar{\gamma}_{1}}{\bar{\gamma}_{0}+\bar{\gamma}_{1}}$.

As a sanity check, we evaluate the previous expression for the special case when the signals belong to the same modulation level $M$ as

$$
B E R=\frac{1}{2} c_{M}\left(1-\sqrt{\frac{d_{M}^{2} \bar{\gamma}_{0}}{1+d_{M}^{2} \bar{\gamma}_{0}}}-\sqrt{\frac{d_{M}^{2} \bar{\gamma}_{1}}{1+d_{M}^{2} \bar{\gamma}_{1}}}+\frac{\sqrt{d_{M}^{2} \bar{\gamma}_{2}}}{\sqrt{1+d_{M}^{2} \bar{\gamma}_{2}}}\right)
$$

where $\bar{\gamma}_{2} \triangleq \frac{\bar{\gamma}_{0} \bar{\gamma}_{1}}{\bar{\gamma}_{0}+\bar{\gamma}_{1}}$. Note that (3.12) is identical to [22, Eq. 9.210], even though they were derived in very different ways. This suggests that [22, Eq. 9.210] can be viewed as a special case of our derived BER expression for SC. 
In Fig. 3.1, we plot the BER performance of $\mathrm{SC}$ for a single relay network, for different average SNRs using numerical simulation as well as the exact BER expression giving in (3.11). It is clear from the figure that there is an excellent agreement between the derived BER expression and the simulation results which validates the mathematical derivations.

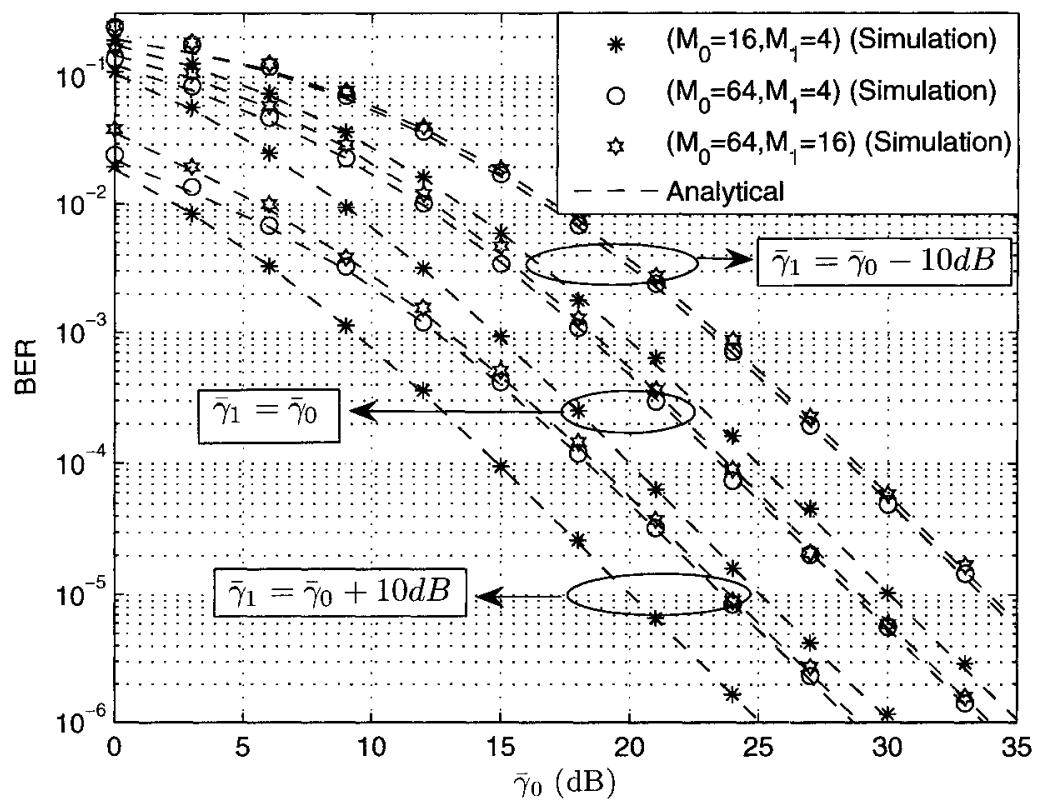

Figure 3.1: BER performance of SC.

\subsection{Performance Analysis of BSC}

The instantaneous BER at the output of BSC, given $\gamma_{0}$ and $\gamma_{1}$, can be written as

$$
B E R_{\text {inst }}=\left\{\begin{array}{l}
c_{M_{0}} Q\left(\sqrt{2 d_{M_{0}}^{2} \gamma_{0}}\right), c_{M_{0}} Q\left(\sqrt{2 d_{M_{0}}^{2} \gamma_{0}}\right) \leq c_{M_{1}} Q\left(\sqrt{2 d_{M_{1}}^{2} \gamma_{1}}\right) \\
c_{M_{1}} Q\left(\sqrt{2 d_{M_{1}}^{2} \gamma_{1}}\right), c_{M_{0}} Q\left(\sqrt{2 d_{M_{0}}^{2} \gamma_{0}}\right)>c_{M_{1}} Q\left(\sqrt{2 d_{M_{1}}^{2} \gamma_{1}}\right)
\end{array}\right.
$$

Similar to the procedure in the previous section, we average (3.13) over the joint PDF of $\gamma_{0}$ and $\gamma_{1}$. However, intervals of the piecewise function in (3.13) contains non-linear 
functions, which makes it difficult to perform the integration. Consequently, we resort to approximating the intervals by linear functions through the use of the following approximation

$$
\left\{\begin{array}{l}
c_{M_{0}} Q\left(\sqrt{2 d_{M_{0}}^{2} \gamma_{0}}\right) \approx Q\left(\sqrt{2 d_{M_{0}}^{2} \gamma_{0}}\right), \gamma_{0}>>1 \\
c_{M_{1}} Q\left(\sqrt{2 d_{M_{1}}^{2} \gamma_{1}}\right) \approx Q\left(\sqrt{2 d_{M_{1}}^{2} \gamma_{1}}\right), \gamma_{1}>>1
\end{array} .\right.
$$

The high accuracy of the previous approximation is due to the exponential nature of the $Q$ function which results in having its argument as the dominant factor. As it will be shown later, such an approximation significantly simplifies the analysis, while sustaining high accuracy.

By utilizing (3.14), the intervals of the piecewise function given in (3.13) can be simplified and the instantaneous BER at the output of BSC can be well-approximated as

$$
B E R_{\text {inst }} \approx\left\{\begin{array}{l}
c_{M_{0}} Q\left(\sqrt{2 d_{M_{0}}^{2} \gamma_{0}}\right), d_{M_{0}}^{2} \gamma_{0} \leq d_{M_{1}}^{2} \gamma_{1} \\
c_{M_{1}} Q\left(\sqrt{2 d_{M_{1}}^{2} \gamma_{1}}\right), d_{M_{0}}^{2} \gamma_{0}>d_{M_{1}}^{2} \gamma_{1}
\end{array}\right.
$$

Using (3.15) and (3.5), the average BER can be well-approximated as

$$
\begin{aligned}
B E R \approx & \int_{0}^{\infty} \int_{0}^{\frac{d_{M_{1}}^{2}}{\bar{d}_{M_{0}}^{2}} \gamma_{1}} c_{M_{1}} Q\left(\sqrt{2 d_{M_{1}}^{2} \gamma_{1}}\right) \frac{1}{\bar{\gamma}_{0}} \frac{1}{\bar{\gamma}_{1}} e^{-\frac{\gamma_{0}}{\bar{\gamma}_{0}}} e^{-\frac{\gamma_{1}}{\bar{\gamma}_{1}}} d \gamma_{0} d \gamma_{1} \\
& +\int_{0}^{\infty} \int_{d_{M_{1}}^{2}}^{\infty} c_{M_{0}} Q\left(\sqrt{2 d_{M_{0}}^{2} \gamma_{0}}\right) \frac{1}{\overline{\bar{\gamma}}_{0}} \frac{1}{\bar{\gamma}_{1}} e^{-\frac{\gamma_{0}}{\bar{\gamma}_{0}}} e^{-\frac{\gamma_{1}}{\bar{\gamma}_{1}}} d \gamma_{0} d \gamma_{1}
\end{aligned}
$$

The integrations in (3.16) can be evaluated using the procedure explained in the previous section; this results in the average BER which can be expressed in terms of 
the functions $H(x ; a, b, c, d)$ and $J(x ; a, b, c, d)$ as

$$
\begin{aligned}
B E R \approx & H\left(\infty ; c_{M_{1}}, d_{M_{1}}^{2}, \bar{\gamma}_{1}\right)-H\left(\infty ; c_{M_{1}} \frac{d_{M_{0}}^{2} \bar{\gamma}_{0}}{d_{M_{0}}^{2} \bar{\gamma}_{0}+d_{M_{1}}^{2} \bar{\gamma}_{1}}, d_{M_{1}}^{2}, \frac{d_{M_{0}}^{2} \bar{\gamma}_{0} \bar{\gamma}_{1}}{d_{M_{0}}^{2} \bar{\gamma}_{0}+d_{M_{1}}^{2} \bar{\gamma}_{1}}\right) \\
& +H\left(\infty ; c_{M_{0}}, d_{M_{0}}^{2}, \bar{\gamma}_{0}\right)-J\left(\infty ; c_{M_{0}}, d_{M_{1}}^{2}, \frac{d_{M_{0}}^{2}}{d_{M_{1}}^{2}} \bar{\gamma}_{0}, \bar{\gamma}_{1}\right)-H\left(0 ; c_{M_{1}}, d_{M_{1}}^{2}, \bar{\gamma}_{1}\right) \\
& +H\left(0 ; c_{M_{1}} \frac{d_{M_{0}}^{2} \bar{\gamma}_{0}}{d_{M_{0}}^{2} \bar{\gamma}_{0}+d_{M_{1}}^{2} \bar{\gamma}_{1}}, d_{M_{1}}^{2}, \frac{d_{M_{0}}^{2} \bar{\gamma}_{0} \bar{\gamma}_{1}}{d_{M_{0}}^{2} \bar{\gamma}_{0}+d_{M_{1}}^{2} \bar{\gamma}_{1}}\right)+J\left(0 ; c_{M_{0}}, d_{M_{1}}^{2}, \frac{d_{M_{0}}^{2}}{d_{M_{1}}^{2}} \bar{\gamma}_{0}, \bar{\gamma}_{1}\right) .
\end{aligned}
$$

Finally, we evaluate the previous expression using (3.7) and (3.8). After considerable simplifications, the average BER can be explicitly approximated as

$$
\begin{aligned}
B E R \approx & \frac{1}{2} c_{M_{0}}\left(1-\sqrt{\frac{d_{M_{0}}^{2} \bar{\gamma}_{0}}{1+d_{M_{0}} \bar{\gamma}_{0}}}\right)+\frac{1}{2} c_{M_{1}}\left(1-c_{M_{1}} \sqrt{\frac{d_{M_{1}}^{2} \bar{\gamma}_{1}}{1+d_{M_{1}}^{2} \bar{\gamma}_{1}}}\right) \\
& -\frac{1}{2} \frac{c_{M_{0} d_{M_{1}}^{2} \bar{\gamma}_{1}+c_{M_{1}} d_{M_{0}}^{2} \bar{\gamma}_{0}}}{d_{M_{0}}^{2} \bar{\gamma}_{0}+d_{M_{1}}^{2} \bar{\gamma}_{1}}\left(1-\sqrt{\frac{\bar{\gamma}_{2}}{1+\bar{\gamma}_{2}}}\right),
\end{aligned}
$$

where $\bar{\gamma}_{2} \triangleq \frac{d_{M_{0}}^{2} \bar{\gamma}_{0} d_{M_{1}}^{2} \bar{\gamma}_{1}}{d_{M_{0}}^{2} \bar{\gamma}_{0}+d_{M_{1}}^{2} \bar{\gamma}_{1}}$. Once again, as a sanity check, we evaluate (3.18) for the special case when the signals to be combined belong to the same modulation level $M$ as

$$
B E R=\frac{1}{2} c_{M}\left(1-\sqrt{\frac{d_{M}^{2} \bar{\gamma}_{0}}{1+d_{M}^{2} \bar{\gamma}_{0}}}-\sqrt{\frac{d_{M}^{2} \bar{\gamma}_{1}}{1+d_{M}^{2} \bar{\gamma}_{1}}}+\sqrt{\frac{\bar{\gamma}_{2}}{1+\bar{\gamma}_{2}}}\right)
$$

where $\bar{\gamma}_{2} \triangleq d_{M}^{2} \frac{\bar{\gamma}_{0} \bar{\gamma}_{1}}{\bar{\gamma}_{0}+\bar{\gamma}_{1}}$. Note that (3.19) is identical to both (3.12) and [22, Eq. 9.210]; this is expected since BER-SC reduces to SNR-SC for this special case.

In Fig. 3.2, we plot the BER performance of BSC for a single relay network, for different average SNRs using numerical simulation as well as the approximation of the BER giving in (3.18). It is clear from the figure that there is an excellent agreement between the derived BER expression and the simulation results which validates the mathematical derivations and justifies the approximations made.

\subsection{Comparison between $\mathrm{SC}$ and BSC}

Although the derived BER for SC and BSC given by (3.11) and (3.18), respectively, are very useful in estimating the BER performance, it is not straightforward 


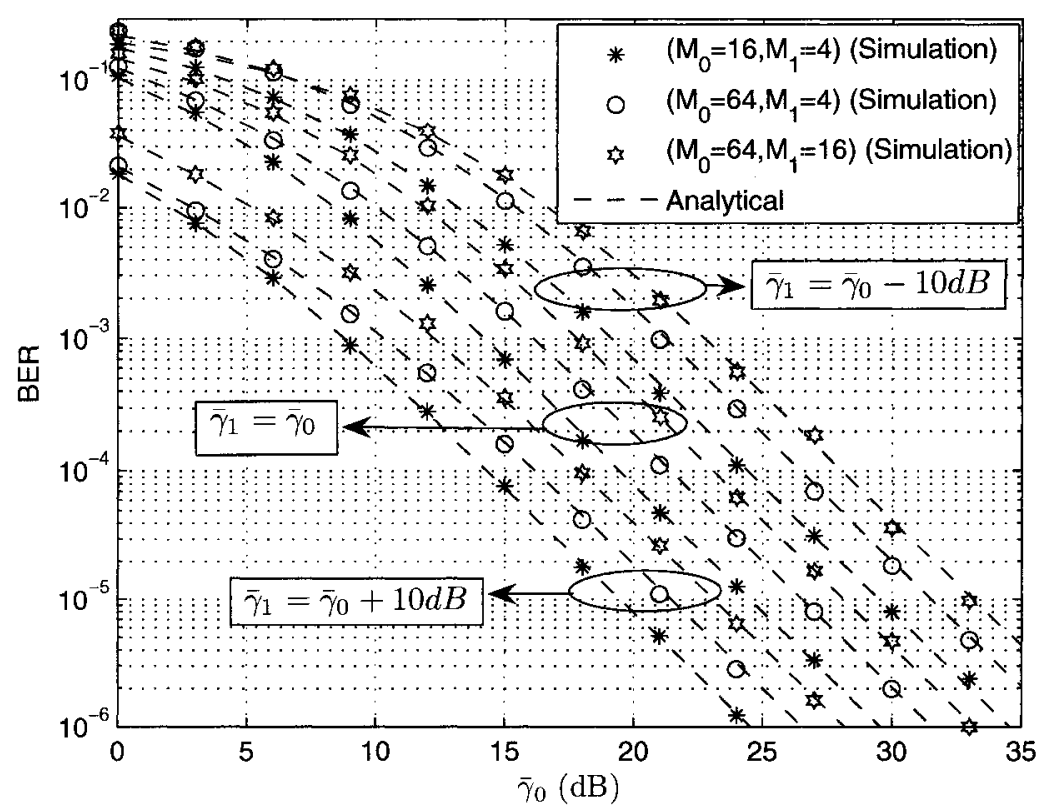

Figure 3.2: BER performance of BSC.

to use them to quantify the gain achieved by using BSC over SC. Consequently, we derive simple asymptotic BER expression for both schemes and we quantify the asymptotic gain of BSC.

We start by writing the average SNRs in the BER expressions given by (3.11) and (3.18) as $\bar{\gamma}_{0}=\sigma_{0}^{2}$ SNR and $\bar{\gamma}_{1}=\sigma_{1}^{2}$ SNR . The goal is to get simple expressions for the BER as SNR goes to infinity. By using Taylor series expansion and truncating the higher order terms, the following asymptotic approximation can be made [23]

$$
1-\sqrt{\frac{x}{x+1}} \approx \frac{1}{2 x}-\frac{3}{8 x^{2}}, \text { as } x \rightarrow \infty
$$

Applying the previous approximation in (3.11) and going through considerable manipulations and simplifications, we get the following asymptotic BER expression for $\mathrm{SC}$

$$
B E R \approx\left(D^{S C} \mathbf{S N R}\right)^{-2}, \text { as } \mathbf{S N R} \rightarrow \infty
$$


where $D^{S C}=\frac{4}{\sqrt{3}}\left(\frac{c_{M_{0}} d_{M_{1}}^{4}+c_{M_{1}} d_{M_{0}}^{4}}{d_{M_{0}}^{4} d_{M_{1}}^{4}}\right)^{-\frac{1}{2}} \sigma_{0} \sigma_{1}$. The constant $D^{S C}$ represents the SNR gain achieved by SC. Similarly, the asymptotic BER expression for BSC can be written as

$$
B E R \approx\left(D^{B S C} \mathbf{S N R}\right)^{-2}, \text { as } \mathbf{S N R} \rightarrow \infty
$$

where $D^{B S C}=\frac{4}{\sqrt{3}}\left(c_{M_{0}}+c_{M_{1}}\right)^{-\frac{1}{2}} d_{M_{0}} d_{M_{1}} \sigma_{0} \sigma_{1}$.

By comparing (3.21) and (3.22), we observe that both schemes achieve diversity order of 2, i.e., full diversity. However, the SNR gain achieved by BSC is higher than that of SC. We define the asymptotic gain (AG) in $\mathrm{dB}$, achieved by BSC over SC as

$$
\begin{aligned}
& A G=10 \log _{10}\left(\frac{D^{B S C}}{D^{S C}}\right)=10 \log _{10}\left(\frac{\frac{4}{\sqrt{3}}\left(c_{M_{0}}+c_{M_{1}}\right)^{-\frac{1}{2}}}{d_{M_{0}} d_{M_{1} \sigma_{0} \sigma_{1}}}\right) \\
&\left.\frac{4}{\sqrt{3}\left(\frac{c_{M_{0}} d_{M_{1}}^{4}+c_{M_{1}}}{d_{M_{0}}^{4} d_{M_{1}}^{4}}\right)^{-\frac{1}{2}} \sigma_{0} \sigma_{1}}\right) \\
&=5 \log _{10}\left(\frac{c_{M_{0}} \frac{d_{M_{1}}^{2}}{d_{M_{0}}^{2}}+c_{M_{1}} \frac{d_{M_{0}}^{2}}{d_{M_{1}}^{2}}}{c_{M_{0}}+c_{M_{1}}}\right) .
\end{aligned}
$$

It is interesting to note that $A G$ is independent of the average SNRs and it merely depends on the modulation levels of the signals to be combined. By substituting (3.3) in (3.23), we evaluate AG for different scenarios as follows

$$
A G=\left\{\begin{array}{l}
0.57 \mathrm{~dB}, \text { combining QPSK and 16-QAM } \\
2.13 \mathrm{~dB}, \text { combining QPSK and 64-QAM } \\
0.77 \mathrm{~dB}, \text { combining 16-QAM and 64-QAM }
\end{array} .\right.
$$

Note that $\mathrm{AG}=0 \mathrm{~dB}$ when $M_{0}=M_{1}=M$, since $\mathrm{SC}$ and $\mathrm{BSC}$ are equivalent in this scenario.

To confirm the accuracy of the asymptotic approximation given by (3.21) and (3.22), and the AG given by (3.24), we plot the exact and the asymptotic BER for different scenarios in Fig. 3.3. It is clear that the asymptotic expression is tight for high SNRs which also confirms the accuracy of the calculated AGs for the different 
scenarios. It is worth repeating that such an asymptotic approximation is used merely for quantifying the gain of BSC over SC and it shouldn't be used as an approximate BER, since such an approximation is loose in the low SNR regime, as shown in the figure.

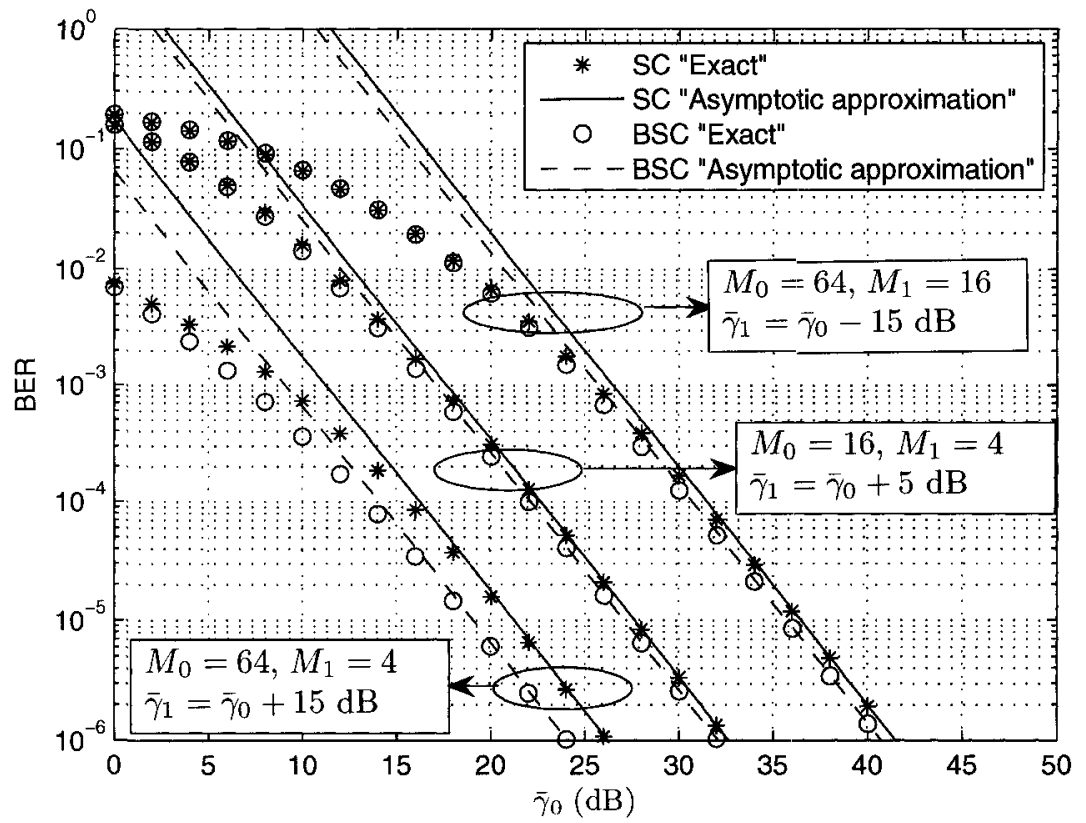

Figure 3.3: BER performance of SC and BSC. 


\section{Chapter 4}

\section{Optimal and Near-Optimal Diversity Combining of Signals with \\ Different Modulation Levels}

Although in the previous chapter we proposed a better selection combining scheme, it is still far from being optimal as it utilizes only one of the received signals. In this chapter, we investigate different receiver structures that achieve the optimal performance. We start by developing the optimal detector as a maximum likelihood detector in Section 4.1. To overcome the complexity of the maximum likelihood detector, we investigate two other receiver structures that perform bit-level combining in Section 4.2 and Section 4.3. Analytical and Simulation results of the BER performance of the different schemes are presented in Section 4.4. Finally, the benefit of combining signals with different modulation levels in term of the end-to-end spectral efficiency is illustrated in Section 4.5.

Notations: For a random variable $X, \bar{X}=E\{X\}$ denotes its mean; $f_{X \mid y=c}(x)$ is the conditional probability density function of $X$ evaluated at $x$ given that $y=c$; for a complex number $B, \mathcal{R}\{B\}$ and $\mathcal{I}\{B\}$ denote the real and imaginary parts of $B$, respectively; $B^{*}$ is the complex conjugate of $B$; for integer numbers $D$ and $E$, $\operatorname{rem}(D, E)$ denotes the remainder of dividing $D$ by $E ; p(\Psi)$ is the probability that event $\Psi$ occurs; $g\left(x ; \mu, \sigma^{2}\right)$ denotes the Gaussian probability density function (PDF) with mean $\mu$ and variance $\sigma^{2}$, i.e., $g\left(x ; \mu, \sigma^{2}\right)=(1 / \sigma \sqrt{2 \pi}) \exp \left(-(x-\mu)^{2} /\left(2 \sigma^{2}\right)\right)$; 
$\mathbf{G}^{N}\left(x ; \boldsymbol{\mu}, \sigma^{2}\right)$ denotes the PDF of a Gaussian mixture random variable that consists of $N$ equal-probable Gaussian random variables with the same variance and different means, i.e., $\mathbf{G}^{N}\left(x ; \boldsymbol{\mu}, \sigma^{2}\right)=\frac{1}{N} \sum_{i=1}^{N} g\left(x, \mu_{i}, \sigma^{2}\right)$, where $\boldsymbol{\mu}=\left[\mu_{1}, \mu_{2}, \ldots, \mu_{N}\right]$.

\subsection{The Optimal Detector: The Maximum Likelihood Detector(MLD)}

\subsubsection{Receiver structure}

The optimum solution to the problem at hand is to use maximum likelihood detection. For additive white Gaussian noise, the MLD reduces to a minimum distance classifier [23]. Consequently, the MLD decides on the sequence $\left\{\hat{s}_{0}, \ldots, \hat{s}_{C-1}\right\}$ that satisfies the following criterion:

$$
\begin{aligned}
& {\left[\hat{s}_{0}, \ldots, \hat{s}_{C-1}\right]=} \\
& \arg \min _{s_{0}, \ldots, s_{C-1}} \sum_{i=0}^{L-1} \sum_{j=0}^{T_{i}-1}\left|r_{i, j}^{M_{i}}-\alpha_{i} S_{i, j}^{M_{i}}\left(s_{j K_{i}}, s_{j K_{i}+1}, \ldots, s_{j K_{i}+\left(K_{i}-1\right)}\right)\right|^{2}
\end{aligned}
$$

The block diagram of the MLD is shown in Fig. 4.1.

From the previous expression, it is imperative to mathematically define the bit to symbol mapping $\left(S_{i, j}^{M_{i}}\left(s_{j K_{i}}, s_{j K_{i}+1}, \ldots, s_{(j+1) K_{i}-1}\right)\right)$ for square M-QAM with Graycoding, which is discussed in the subsequent section.

\subsubsection{Square M-QAM modulation with Gray-Coding}

For a given sequence of bits $\left\{s_{0}, s_{1}, \ldots, s_{K-1}\right\}$, where $s_{i} \in\{-1,1\}$, we develop a mathematical model that maps these $K$ bits into a Gray-coded $M$-QAM symbol, $S^{M}$. The symbol $s_{0}$ denotes the most-significant bit while the symbol $s_{K-1}$ denotes the least-significant bit. We start by considering particular 4-QAM (QPSK) and 16QAM with Gray-Coding, and then we generalize the model for any square M-QAM constellation with Gray-coding. For the QPSK constellation shown in Fig. 4.2, it is 


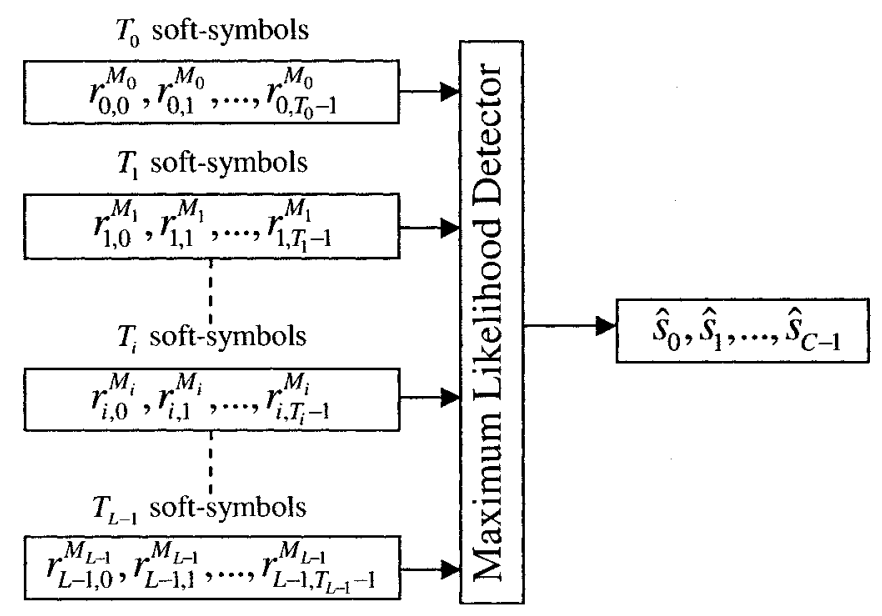

Figure 4.1: The MLD block diagram.

not difficult to find that

$$
S^{4}=s_{0} d_{4}-j s_{1} d_{4}
$$

where $d_{M}$ is a constant that is used to make the average energy per bit for the M-QAM constellation equal to unity and it is given by ${ }^{12}$

$$
d_{M_{i}}=\sqrt{\frac{3 \log _{2} M_{i}}{2\left(M_{i}-1\right)}}
$$

However, for the 16-QAM constellation depicted in Fig. 4.3, it needs more effort to express $S_{16}$ as a function of $\left\{s_{0}, s_{1}, \ldots, s_{3}\right\}$. It is shown in [25] that the only possible way to construct Gray coded M-QAM constellation is by the cross product of two Gray coded PAM constellations. Consequently, the problem is simplified to finding the relationship between $\mathcal{R}\left\{S^{16}\right\}$ and the sequence $\left\{s_{0}, s_{1}\right\}$, and finding the relationship between $\mathcal{I}\left\{S^{16}\right\}$ and the sequence $\left\{s_{2}, s_{3}\right\}$. We start by seeking a

${ }^{1}$ Since the reference SNR in this thesis is in terms of $\mathrm{Eb} / \mathrm{N} 0$, the average energy per bit is fixed to unity.

${ }^{2}$ To have the reference SNR in terms of Es/NO, the average energy per symbol needs to be fixed to unity. This can be done by expressing $d_{M_{i}}$ as $d_{M_{i}}=\sqrt{\frac{3}{2\left(M_{i}-1\right)}}$, and the derived equations in this thesis still apply for this case. 


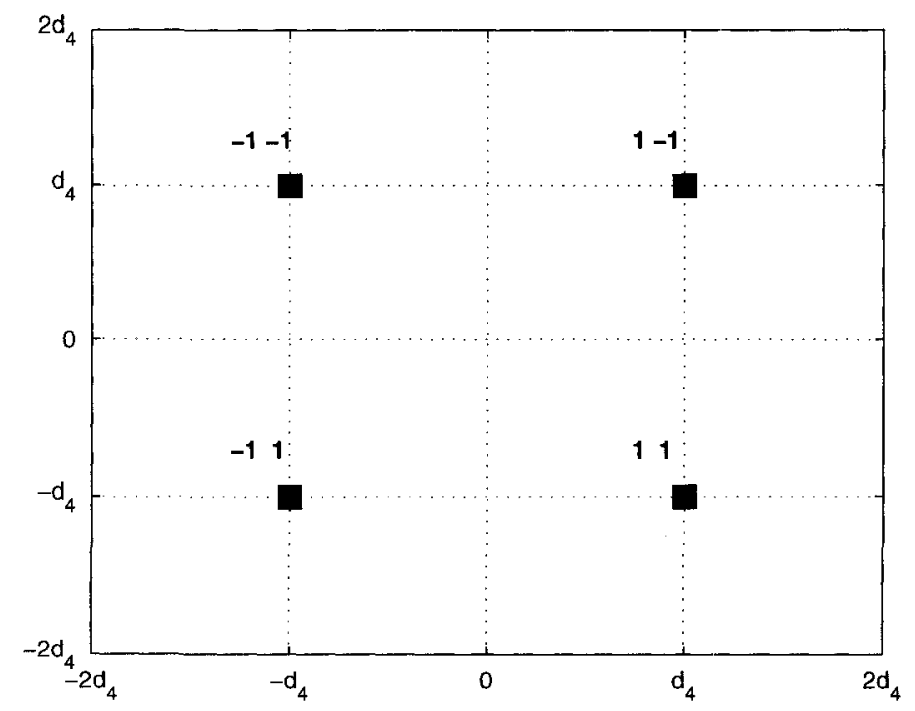

Figure 4.2: 4-QAM (QPSK) constellation with Gray-coding

linear relationship between $\mathcal{R}\left\{S^{16}\right\}$ and $\left\{s_{0}, s_{1}\right\}$. In other words, we need to find the coefficients $x_{0}$ and $x_{1}$ that satisfies the following equation:

$$
\mathcal{R}\left\{S^{16}\right\}=x_{0} s_{0}+x_{1} s_{1}
$$

for all possible combination of $s_{0}$ and $s_{1}$. For the labeling depicted in Fig. 4.3, the previous equation can be written as

$$
\mathbf{A x}=\mathbf{b}, \text { where } \mathbf{A}=\left[\begin{array}{cc}
-1 & -1 \\
-1 & 1 \\
1 & -1 \\
1 & 1
\end{array}\right], \mathbf{x}=\left[\begin{array}{l}
x_{0} \\
x_{1}
\end{array}\right], \text { and } \mathbf{b}=\left[\begin{array}{c}
-3 d_{16} \\
-d_{16} \\
+3 d_{16} \\
+d_{16}
\end{array}\right]
$$

Since $\mathrm{A}$ is a $4 \times 2$ matrix with rank 2 , this is an overdetermined system of linear equations where a solution does not exist. In other words, we have more linearly independent equations than unknowns. We conclude that it is not possible to find a linear mapping that maps $\left\{s_{0}, s_{1}\right\}$ to $\mathcal{R}\left\{S_{16}\right\}$. Consequently, we resort to non-linear 
mapping. In non-linear mapping, we solve for the coefficients $x_{0}, x_{1}, x_{2}$, and $x_{3}$ that satisfy the following equation:

$$
\mathcal{R}\left\{S^{16}\right\}=x_{0} s_{0}+x_{1} s_{1}+x_{2} s_{0} s_{1}+x_{3}
$$

for all possible combination of $s_{0}$ and $s_{1}$. For the labeling depicted in Fig. 4.3, the previous equation can be written as follows:

$$
\mathbf{A} \mathbf{x}=\mathbf{b} \text {, where } \mathbf{A}=\left[\begin{array}{cccc}
-1 & -1 & 1 & 1 \\
-1 & 1 & -1 & 1 \\
1 & -1 & -1 & 1 \\
1 & 1 & 1 & 1
\end{array}\right], \mathbf{x}=\left[\begin{array}{c}
x_{0} \\
x_{1} \\
x_{2} \\
x_{3}
\end{array}\right] \text {, and } \mathbf{b}=\left[\begin{array}{c}
-3 d_{16} \\
-d_{16} \\
+3 d_{16} \\
+d_{16} .
\end{array}\right]
$$

Since $\mathrm{A}$ is a full rank $4 \times 4$ matrix, a unique solution does exists. Solving this equation leads us to the following solution:

$$
\mathbf{x}=\left[2 d_{16} 0-d_{16} 0\right]^{T}
$$

As a result, we can write

$$
\mathcal{R}\left\{S^{16}\right\}=2 d_{16} s_{0}-d_{16} s_{0} s_{1}=s_{0}\left(-s_{1}+2\right) d_{16}
$$

Following similar procedure, we can also write

$$
\mathcal{I}\left\{S^{16}\right\}=-s_{2}\left(-s_{3}+2\right) d_{16}
$$

Finally, for this particular case of Gray 16-QAM constellation, one can write $\left\{S^{16}\right\}$ as a function of $\left\{s_{0}, s_{1}, \ldots, s_{3}\right\}$ as

$$
S^{16}=s_{0}\left(-s_{1}+2\right) d_{16}-j s_{2}\left(-s_{3}+2\right) d_{16}
$$

We remark that our model is not necessarily unique, but perhaps as simple as it could be. Even though we derived the model for a particular Gray labeling, the 
same model can be used to generate different Gray coded 16-QAM constellations by either re-labeling the bits, or by negating the sign of the bits. For example, different Gray coded 16-QAM constellations are shown in Appendix B with their mathematical models.

Following similar procedure, the relationship for the 64-QAM constellation shown in Fig. 4.4 can be written as

$$
S^{64}=s_{0}\left(-s_{1}\left(-s_{2}+2\right)+4\right) d_{64}-j s_{3}\left(-s_{4}\left(-s_{5}+2\right)+4\right) d_{64} .
$$

By inspecting the equations (4.2), (4.4), and (4.12), it is easy to see that there is consistency in the model. This consistency is exploited to generalize the model for any Gray coded square M-QAM modulation. The general model is recursive and it is expressed as

$$
S^{M_{i}}\left(s_{0}, s_{1}, \ldots, s_{K_{i}-1}\right)=\left(-\chi_{K_{i} / 2-1}+j \beta_{K_{i} / 2-1}\right) d_{M_{i}}
$$

where $d_{M_{i}}$ is a constant used to fix the energy per bit to unity and it is given by (4.3). For example, $d_{4}=1, d_{16}=0.6325$, and $d_{64}=0.378$.

The coefficients $\chi_{K / 2-1}$ and $\beta_{K / 2-1}$ can be computed recursively as

$$
\begin{gathered}
\chi_{k}=\left\{\begin{array}{cc}
-s_{K / 2-1} & k=0 \\
-s_{K / 2-k-1}\left(\chi_{k-1}+2^{k}\right) & 0<k \leq K / 2-1
\end{array} .\right. \\
\beta_{k}=\left\{\begin{array}{cc}
-s_{K-1} & k=0 \\
-s_{K-k-1}\left(\beta_{k-1}+2^{k}\right) & 0<k \leq K / 2-1
\end{array}\right.
\end{gathered}
$$

Again, this model can be used to generate different possible Gray coded square M-QAM constellations by either re-labeling the bits, or by negating the sign of the bits. 
Similar mathematical model was developed independently in [26, Eq. 3.2] using different approach. Nevertheless, the expression in [26, Eq. 3.2] is limited to a particular Gray mapping.

As stated in the previous section, (4.13) is essential for the MLD described by (4.1)

\subsubsection{Advantages and disadvantages of the MLD}

Although MLD achieves the optimum performance, it has exorbitant computational complexity. For example, to perform the decoding in the case where $M_{0}=4$ $\left(K_{0}=2\right), M_{1}=16\left(K_{1}=4\right)$, and $M_{2}=64\left(K_{2}=6\right)$, the MLD decodes $C=\operatorname{LCM}(2,4,6)=$ 12 bits jointly. This requires at least $2^{12}$ computations, which is clearly too complex. In general, the MLD requires $\frac{2^{C}}{C}$ computations per bit, which means that its complexity grows exponentially with $C$. For this reason, we are motivated to investigate practical schemes with much reduced complexity and with performance comparable to that of the MLD. These schemes are described in the subsequent sections. 


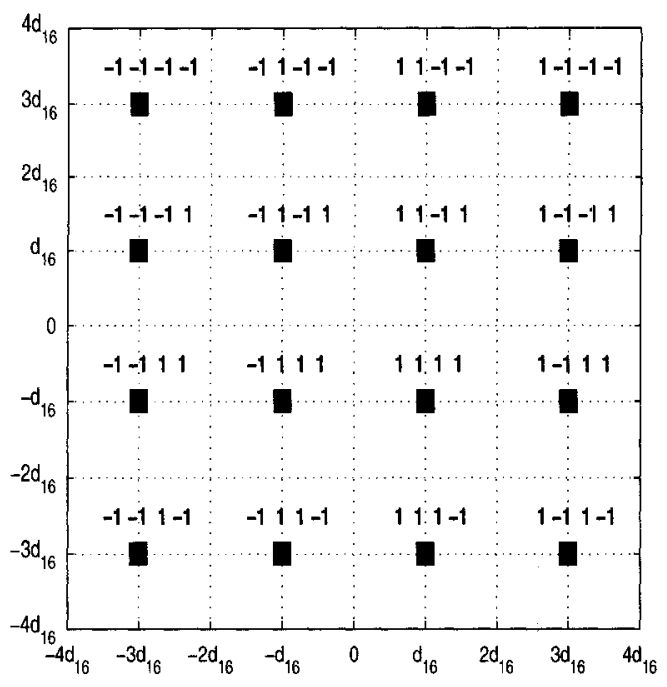

Figure 4.3: 16-QAM constellation with Gray-coding.

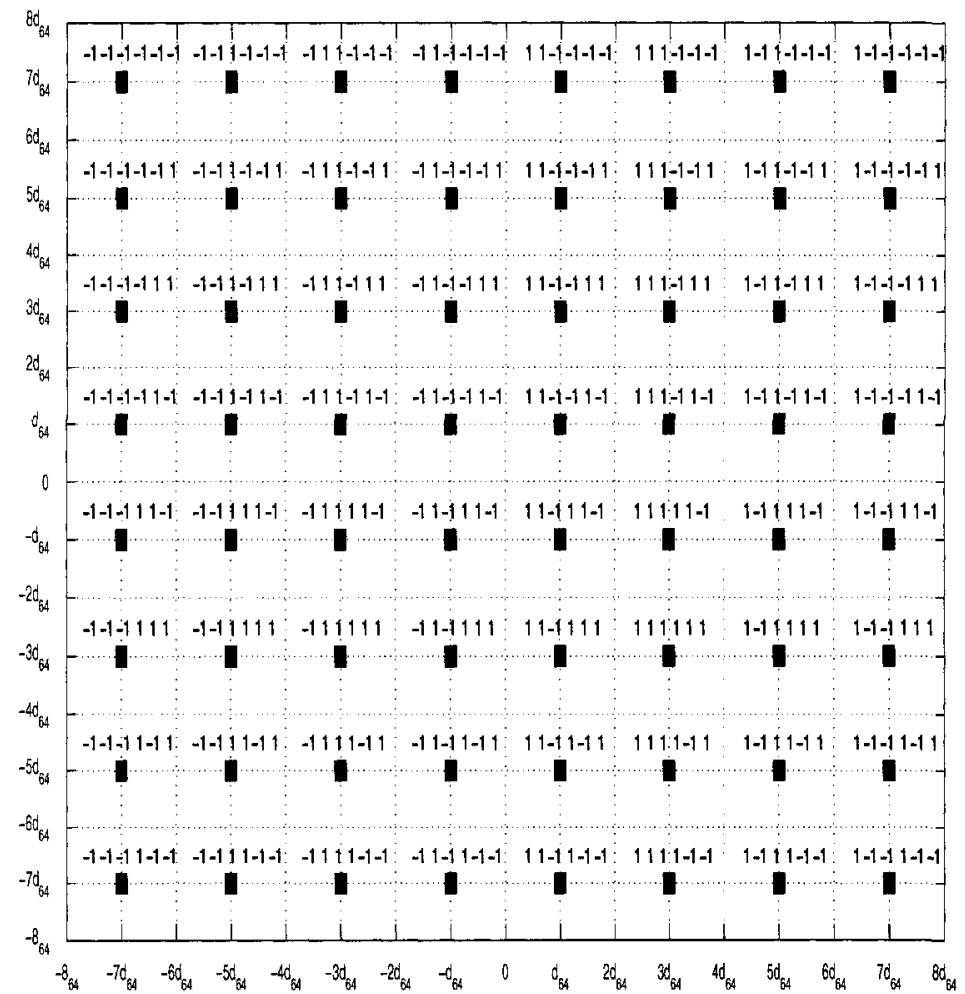

Figure 4.4: 64-QAM constellation with Gray-coding. 


\subsection{Soft-Bit Maximum Likelihood Detector (SBMLD)}

In the MLD, the complexity arises from the fact that different modulation levels carry different number of bits per symbol. As a result, bit-by-bit (or symbol-bysymbol) decoding is not possible. A trivial remedy to this problem is to decode the bits from each link and perform diversity combing on the hard bits. However, it is found out through simulation that this solution does not achieve the full diversity potential because of the lost soft information in the hard decoding. In order to avoid losing the soft-bit information, the received $M_{i}$-QAM soft symbol, $r_{i, j}^{M_{i}}$, is mapped into $K_{i}$ soft-bits. Then, decoding can be performed on the soft-bits, which results in bit-by-bit detection, rather than detecting a sequence of bits jointly. To extract soft-bits from a soft-symbol, the logarithm of likelihood ratio (LLR) can be used. The LLR is a well known technique and it is being used in many channel coding schemes (see for example [27] and [28]). It is also used in the context of HARQ in [14] for diversity combining of signals that belong to the same modulation level, but with different bit to symbol mapping. Combining was performed by adding the soft-bits. In this work, we use the same LLR concept and explain how to use this concept to get close to optimum performance in combining signals with different modulations levels.

Since the computation of the LLRs can be pricey [27], good approximations of the LLRs are used. For the Gray coded $M_{i}$-QAM schemes described by (4.13), the 
LLR can be well approximated by the following recursive expression [27-29]

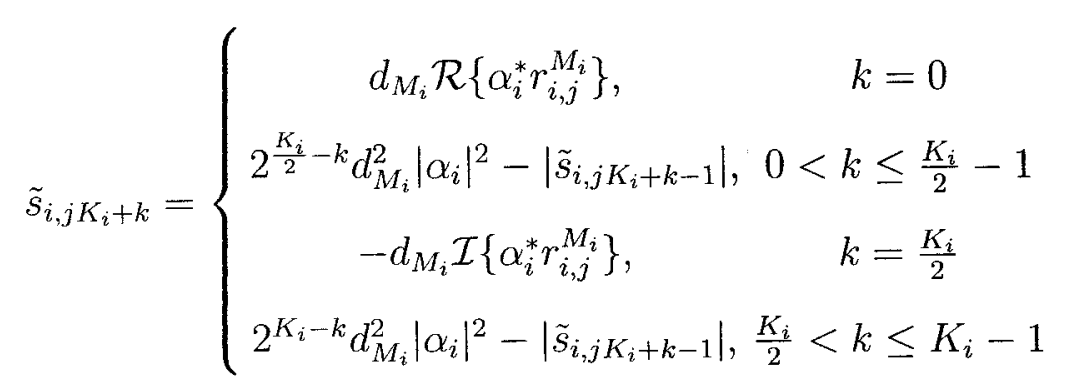

where $\tilde{s}_{i, j K_{i}+k}$ is the $\left(j K_{i}+k\right)^{t h}$ soft-bit generated from the received soft symbol $r_{i, j}^{M_{i}}$. In other words, $k$ denotes the position of the soft-bit in the soft-symbol. Note that multiplying the received soft symbol by the conjugate of the channel coefficient is necessary to undo the phase rotation caused by the channel.

Note that if exact LLR expression is used to calculate the soft-bits, then the optimal detection is done through adding the LLRs (MAP detector). Such a receiver will achieve the minimum possible BER but the complexity associated with exact LLR calculations can be even higher than the MLD.

The SBMLD, which is a bit-by-bit detector, performs maximum likelihood detection on the soft-bits. The SBMLD is the optimum receiver structure that performs detection based on the approximated LLRs (soft-bits). It decides on the bit $\hat{s}_{l}$, for $l \in\{0,1, \ldots, C-1\}$, according to the following criterion

$$
\left\{\begin{array}{cc}
\hat{s}_{l}=1, & f_{\tilde{s}_{0, l}, \ldots, \tilde{s}_{L-1, l} \mid s_{l}=1}\left(\tilde{s}_{0, l}, \ldots, \tilde{s}_{L-1, l}\right)> \\
& f_{\tilde{s}_{0, l}, \ldots, \tilde{s}_{L-1, l} \mid s_{l}=-1}\left(\tilde{s}_{0, l}, \ldots, \tilde{s}_{L-1, l}\right) \\
\hat{s}_{l}=-1, & \text { otherwise }
\end{array} .\right.
$$

Note that in the above $l=j K_{i}+k$. The block diagram of this scheme is shown in Fig. 4.5.

Since the symbols received from different nodes are assumed to be statistically independent, the joint conditional PDF is the multiplication of the marginal condi- 


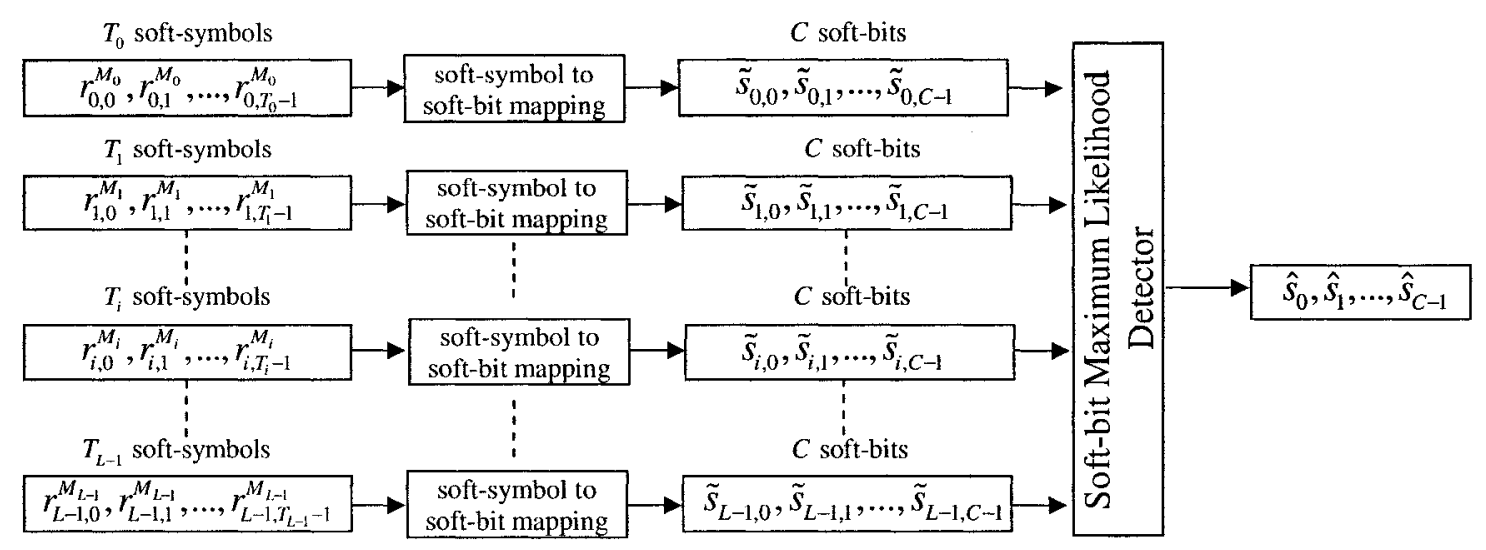

Figure 4.5: The SBMLD block diagram.

tional PDFs. Hence, (4.16) reduces to

$$
\left\{\begin{array}{c}
\hat{s}_{l}=1, \text { if } \prod_{i=0}^{L-1} f_{\tilde{s}_{i, l} \mid s_{l}=1}\left(\tilde{s}_{i, l}\right)>\prod_{i=0}^{L-1} f_{\tilde{s}_{i, l} \mid s_{l}=-1}\left(\tilde{s}_{i, l}\right) \\
\hat{s}_{l}=-1, \quad \text { otherwise }
\end{array} .\right.
$$

Since calculating the soft-bits costs one simple computation per bit, the SBMLD requires $L C / C=L$ computations per bit. This means that the SBMLD's complexity grows linearly with $L$ as opposed to the MLD which has complexity growing exponentially with $C$.

From (4.15), it is easy to see that the marginal conditional PDFs of $\tilde{s}_{i, j K_{i}+k}$ are the same as those of $\tilde{s}_{i, k}$, for $j=0,1, \ldots, T_{i}-1$. Furthermore, from the similarity of the expression of the bits in the real and imaginary parts, it can be easily shown that the conditional PDF expression of the soft-bit $\tilde{s}_{i, k}$ is the same as that of $\tilde{s}_{i, k-K_{i} / 2}$, for $k \geq K_{i} / 2$. Thus, it suffices to provide the conditional PDFs expression of $\tilde{s}_{i, k_{i}}$ for $k_{i} \in\left\{0,1, \ldots, K_{i} / 2-1\right\}$, where $k_{i}=\operatorname{rem}\left(l, K_{i} / 2\right)$.

The conditional PDF of the soft-bits can be obtained from (4.15) by using the following properties that apply to any random variable $X$ and constant $c$

1) $f_{|X|}(x)=\left\{\begin{array}{cc}f_{X}(x)+f_{X}(-x), & x \geq 0 \\ 0, & x<0\end{array}\right.$ 
2) $f_{X+c}(x)=f_{X}(x-c)$.

The conditional PDFs of the soft-bits are given by

$$
\begin{aligned}
& f_{\tilde{s}_{i, k_{i}} \mid s_{n}= \pm 1}(x)= \\
& \left\{\begin{array}{cc}
\mathrm{G}^{\sqrt{M_{i} / 2}}\left(x ; \lambda_{n}^{ \pm} d_{M_{i}}\left|\alpha_{i}\right|^{2}, d_{M_{i}}^{2}\left|\alpha_{i}\right|^{2} N_{0} / 2\right), & k_{i}=0 \\
f_{\tilde{s}_{i, k_{i}-1} \mid s_{i, n}= \pm 1}\left(x-\eta_{i, k_{i}}\right)+f_{\tilde{s}_{i, k_{i}-1} \mid s_{i, n}= \pm 1}\left(-x+\eta_{i, k_{i}}\right), & 0<k_{i} \leq \frac{K_{i}}{2}-1, x \leq \eta_{i, k_{i}} \\
0, & 0<k_{i} \leq \frac{K_{i}}{2}-1, x>\eta_{i, k_{i}}
\end{array}\right.
\end{aligned}
$$

where $\eta_{i, k_{i}}=2^{\frac{K_{i}}{2}-k_{i}} d_{M_{i}}^{2}\left|\alpha_{i}\right|^{2}$ and $n \in\left\{0,1, \ldots, K_{i} / 2-1\right\}$. The vector $\boldsymbol{\lambda}_{n}^{ \pm}$contains all the possible values of $\mathcal{R}\left\{S^{M_{i}}\right\}$ given that $s_{n}= \pm 1$. The matrices $\lambda^{ \pm}$, where $\boldsymbol{\lambda}^{ \pm}=\left[\boldsymbol{\lambda}_{0}^{ \pm} \ldots \boldsymbol{\lambda}_{K_{i} / 2-1}^{ \pm}\right]$are tabulated in Table 4.1 for the modulation schemes described by (4.13).

For high SNR, where $\left|\alpha_{i}\right|^{2} / N_{0}>>1$, the truncated part of the PDF (caused by the absolute value operation in (4.15) where $\tilde{s}_{i, k_{i}}>\eta_{i, k_{i}}$ ) becomes negligible. Applying this approximation and evaluating the recursive expression in (4.18) yields the following approximation

$$
f_{\tilde{s}_{i, k_{i}} \mid s_{k_{i}}= \pm 1}(x) \approx \mathbf{G}^{N_{i, k_{i}}}\left(x ; \pm \boldsymbol{\mu}_{i, k_{i}}, d_{M_{i}}^{2}\left|\alpha_{i}\right|^{2} N_{0} / 2\right)
$$

where $\boldsymbol{\mu}_{i, k_{i}}=\left\{(2 m+1)\left|\alpha_{i}\right|^{2} d_{M_{i}}^{2}: m \in\left\{0, \ldots, N_{i, k_{i}}-1\right\}\right\}$ and $N_{i, k_{i}}=\sqrt{M_{i}} / 2^{k_{i}+1}$.

Equation (4.19) shows that the conditional PDF of the soft-bits is well approximated as a Gaussian mixture and the number of components of the mixture $N_{i, k}$ depends on the position of the soft-bit $k_{i}$ and the modulation level $M_{i}$. This approximation becomes useful in the subsequent section.

Note that the detection rule in this case didn't simplify to a minimum distance classifier as in the case of the MLD, because the conditional PDFs of the soft-bits are Gaussian mixtures and not Gaussian. 
The conditional PDF's for the soft-bits generated from a soft 16-QAM symbol and a soft 64-QAM symbol are shown in the Figures 4.6 to 4.10 . The simulation results, perfectly match the exact expression. In all figures, the approximation of the conditional PDF given by (4.19) agrees very well with the exact expression for $\gamma_{i}=$ $10 \mathrm{~dB}$ and $\gamma_{i}=20 \mathrm{~dB}$. The approximation becomes looser in Figures 4.7, 4.9, and 4.10 for $\gamma_{i}=0 \mathrm{~dB}$. Nevertheless, the left-tail of the PDF, is still in good agreement. Since the region under the left tail is what determines the BER, approximating the conditional pdf to be Gaussian mixture will introduce only marginal error if it is used in deriving BER expression. 
Table 4.1: The matrices $\boldsymbol{\lambda}^{+}$and $\boldsymbol{\lambda}^{-}$for different M-QAM constellations.

\begin{tabular}{|c|c|c|}
\hline $\begin{array}{c}\text { Modulation } \\
\text { Level }\left(M_{i}\right)\end{array}$ & $\lambda^{+1}$ & $\lambda^{-1}$ \\
\hline 4 & $\lambda^{+1}=d_{4}$ & $\lambda^{-1}=-d_{4}$ \\
\hline 16 & $\lambda^{+1}=\left[\begin{array}{cc}1 & -1 \\
3 & 1\end{array}\right] d_{16}$ & $\lambda^{-1}=\left[\begin{array}{cc}-1 & -3 \\
-3 & 3\end{array}\right] d_{16}$ \\
\hline \multirow{2}{*}{64} & $\lambda^{+1}=\left[\begin{array}{ccc}1 & -1 & -3 \\
3 & 1 & 3 \\
5 & -3 & -5 \\
7 & 3 & 5\end{array}\right] d_{64}$ & $\lambda^{-1}=\left[\begin{array}{ccc}-1 & -5 & -1 \\
-3 & 5 & 1 \\
-5 & -7 & -7 \\
-7 & 7 & 7\end{array}\right] d_{64}$ \\
\hline
\end{tabular}

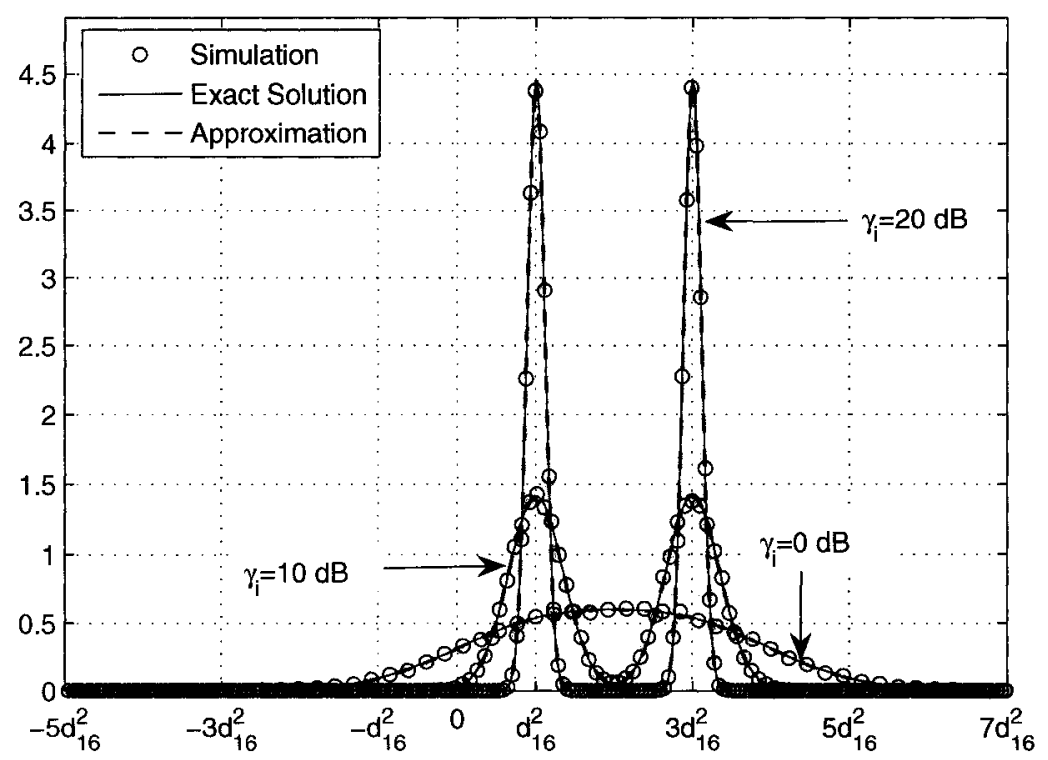

Figure 4.6: Conditional PDF of $\tilde{s}_{i, 0}\left(\tilde{s}_{i, 2}\right)$ generated from a 16-QAM symbol given that $s_{0}=1\left(s_{2}=1\right)$. 


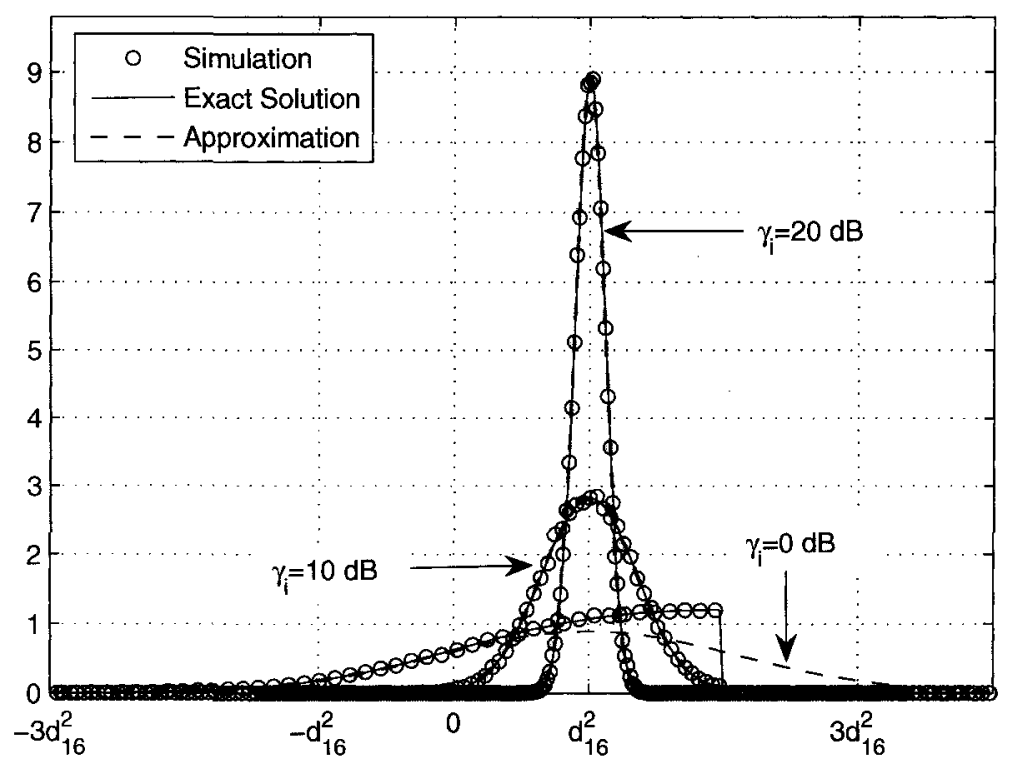

Figure 4.7: Conditional PDF of $\tilde{s}_{i, 1}\left(\tilde{s}_{i, 3}\right)$ generated from a 16-QAM symbol given that $s_{1}=1\left(s_{3}=1\right)$.

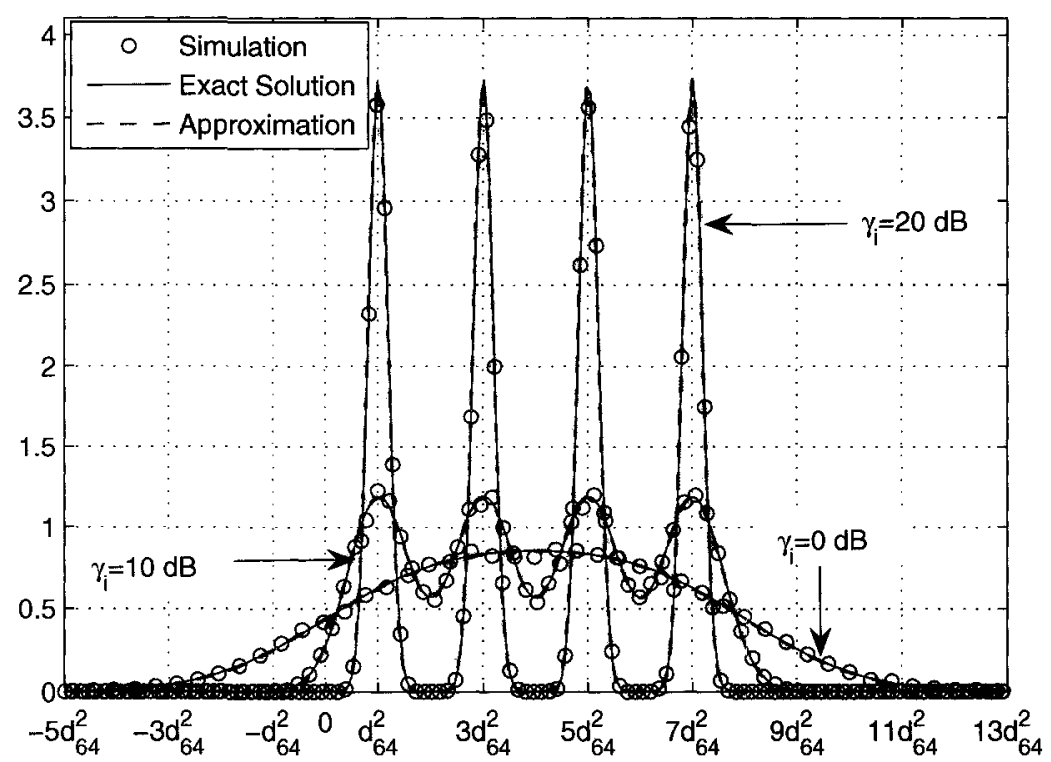

Figure 4.8: Conditional PDF of $\tilde{s}_{i, 0}\left(\tilde{s}_{i, 3}\right)$ generated from a 64-QAM symbol given that $s_{0}=1\left(s_{3}=1\right)$. 


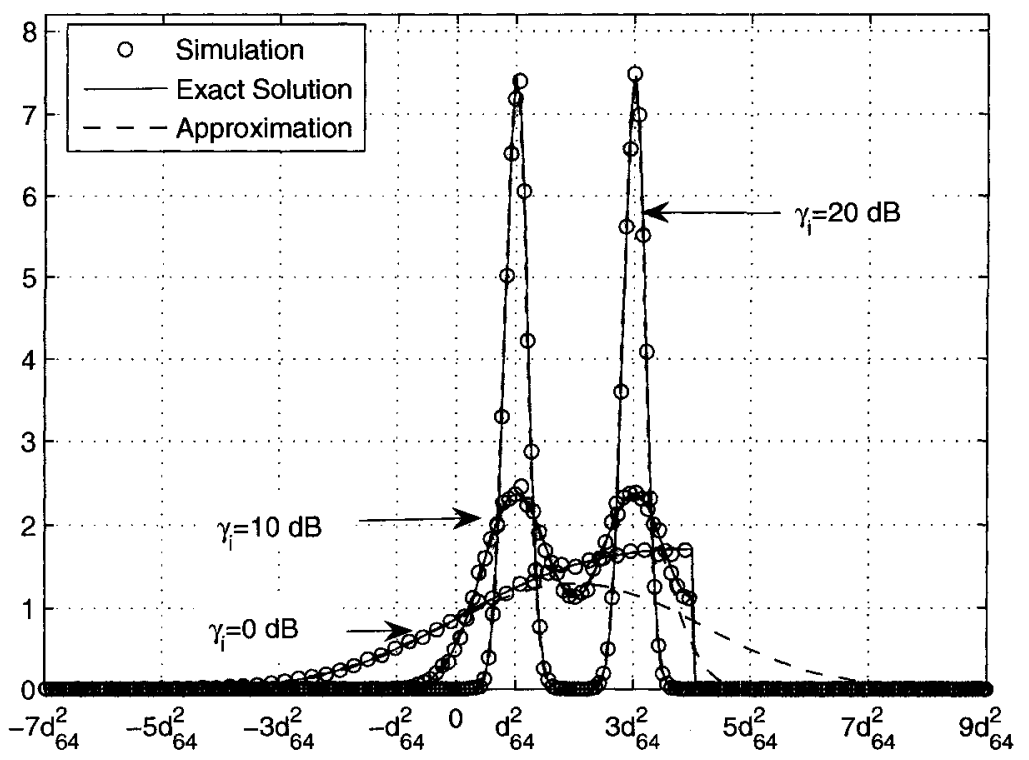

Figure 4.9: Conditional PDF of $\tilde{s}_{i, 1}\left(\tilde{s}_{i, 4}\right)$ generated from a 64-QAM symbol given that $s_{1}=1\left(s_{4}=1\right)$.

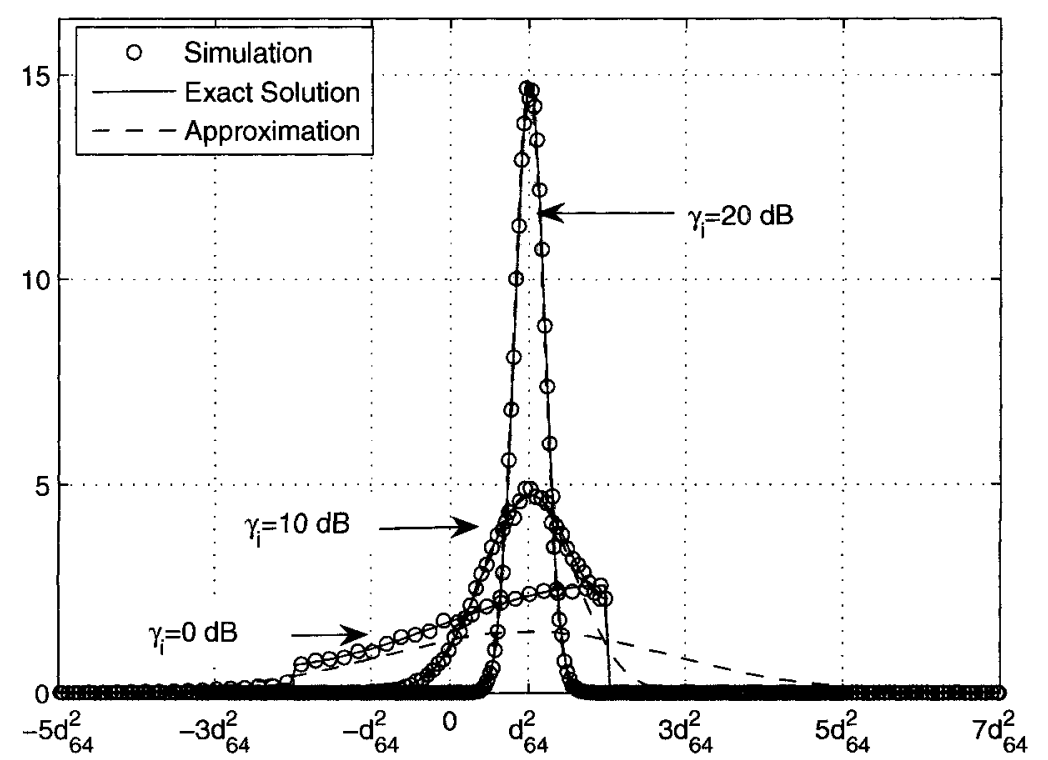

Figure 4.10: Conditional PDF of $\tilde{s}_{i, 2}\left(\tilde{s}_{i, 5}\right)$ generated from a 64-QAM symbol given that $s_{2}=1\left(s_{5}=1\right)$. 


\subsection{Soft-Bit Maximal Ratio Combiner (SBMRC)}

To avoid the computation of the conditional PDFs in the detection process, and to have a scheme that has complexity comparable to the classical MRC, we investigate another scheme which we refer to as the SBMRC.

The SBMRC simply adds the soft bits in a way similar to MRC, hence the name SBMRC. In other words, the SBMRC decides on the bit $\hat{s}_{l}$, for $l \in\{0,1, \ldots, C-1\}$, according to the following criterion

$$
\left\{\begin{array}{c}
\hat{s}_{l}=1 \quad \text { if } \bar{s}_{l}>0 \\
\hat{s}_{l}=-1 \text { otherwise }
\end{array}\right.
$$

where $\bar{s}_{l}$ is the sum of the soft-bits received from different links and is given by ${ }^{3}$

$$
\bar{s}_{l}=\sum_{i=0}^{L-1} \tilde{s}_{i, l}
$$

The soft-bits are already weighted according to their channel conditions by the definition in (4.15), therefore there is no need to weight them again in the combining. The suboptimality of SBMRC comes as result of having approximated LLRs rather than exact LLRs. The block diagram of this scheme is shown in Fig. 4.11.

Similar to the SBMLD, the SBMRC requires $L$ computations per bit. However, processing the soft-bits is used through simple addition, in contrast to the SBMLD that requires evaluation of the conditional PDF of the soft-bits. This means that the SBMRC has lower complexity than the SBMLD, but at the cost of some performance loss. As it will be shown later, this loss in performance is negligible.

The structure of SBMRC does not only reduce the detection complexity but also

${ }^{3}$ In $[30,31]$, we multiply the soft-bits by $d_{M_{i}}$ before combining. In this thesis, $d_{M_{i}}$ is already included in the definition of the soft-bits given by (4.15), to accurately approximate the LLR. Thus, the results reported in $[30,31]$ are still correct and identical to the results in this thesis. 


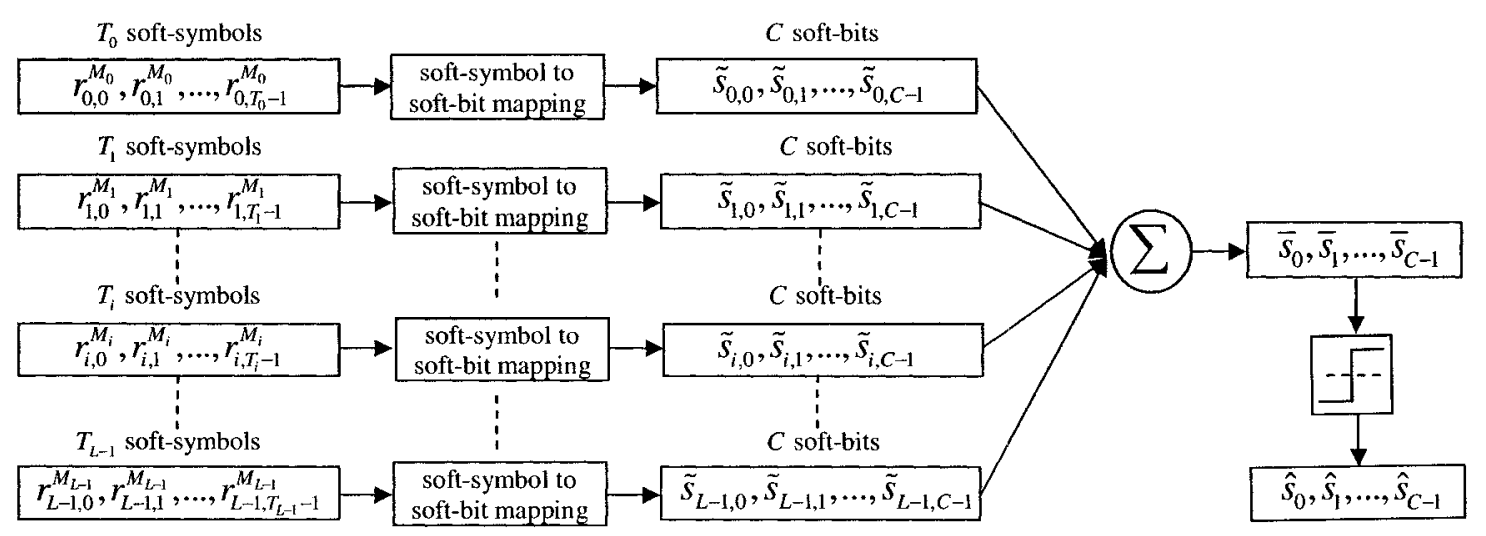

Figure 4.11: The SBMRC block diagram

makes it mathematically tractable to find a closed-form approximation of the BER, in contrast to both MLD and SBMLD.

To find the BER, we start in Section 4.3 .1 by finding $f_{\bar{s}_{l} \mid s_{l}= \pm 1}(x)$ using the approximation of $f_{\tilde{s}_{i, k_{i}} \mid s_{k_{i}}= \pm 1}(x)$ given by (4.19). Then, in Section 4.3 .2 we develop tight bounds on the instantaneous BER. Finally, in Section 4.3.3 we develop tight bounds on the average BER by averaging over the PDFs of the SNRs.

\subsubsection{Finding $f_{\bar{s}_{l} \mid s_{l}= \pm 1}(x)$}

Proposition 1. Let $X_{i}$, for $i \in\{0, \ldots, L-1\}$, be a set of independent random variables, each with a marginal PDF of $f_{X_{i}}\left(x_{i}\right)=\mathbf{G}^{N_{i}}\left(x_{i}, \boldsymbol{\mu}_{i}, \sigma_{i}^{2}\right)$, where $\boldsymbol{\mu}_{i}=\left\{\mu_{i, 0}, \ldots, \mu_{i, N_{i}-1}\right\}$. The PDF of $Z$, where $Z=\sum_{i=0}^{L-1} X_{i}$, is $f_{Z}(z)=\mathrm{G}^{N_{Z}}\left(z, \boldsymbol{\mu}_{z}, \sigma_{z}^{2}\right)$. The parameters of the PDF are defined as follows: $N_{Z}=\prod_{i=0}^{L-1} N_{i}, \sigma_{Z}^{2}=\sum_{i=0}^{L-1} \sigma_{i}^{2}$ and $\boldsymbol{\mu}_{Z}$ is a $1 \times N_{Z}$ vector that consists of the $N_{Z}$ summands in the expression $\sum_{j_{0}=0}^{N_{0}-1} \ldots \sum_{j_{L-1}=0}^{N_{L-1}} \pi_{j_{0}, j_{1}, \ldots, j_{L-1}}$ where $\pi_{j_{0}, j_{1}, \ldots, j_{L-1}}=\sum_{i=0}^{L-1} \mu_{i, j_{i}}$

Proof. As stated in [32], the pdf of the sum of independent random variables is the 
convolution of the individual pdf's. That is

$$
\begin{aligned}
& f_{Z}(z)=\mathbf{G}^{N_{0}}\left(x_{0}, \mu_{0}, \sigma_{0}^{2}\right) \otimes \ldots \otimes \mathbf{G}^{N_{L-1}}\left(x_{L-1}, \mu_{L-1}, \sigma_{L-1}^{2}\right) \\
& f_{Z}(z)=\frac{1}{N_{0}} \sum_{j_{0}=0}^{N_{0}-1} g\left(x_{0} ; \mu_{0, j_{0}}, \sigma_{0}^{2}\right) \otimes \ldots \otimes \frac{1}{N_{L-1}} \sum_{j_{L-1}=0}^{N_{L-1}} g\left(x_{L-1} ; \mu_{i, j_{L-1}}, \sigma_{L-1}^{2}\right) \\
& f_{Z}(z)=\frac{1}{N_{0} \ldots N_{L-1}} \sum_{j_{0}=0}^{N_{0}-1} \ldots \sum_{j_{L-1}=0}^{N_{L-1}} g\left(x_{0} ; \mu_{0, j_{0}}, \sigma_{0}^{2}\right) \otimes \ldots \otimes g\left(x_{L-1} ; \mu_{L-1, j_{L-1}}, \sigma_{L-1}^{2}\right)
\end{aligned}
$$

Since the Gaussian function is closed under convolution, the previous expression can be further simplified to

$$
f_{Z}(z)=\left(\prod_{i=0}^{L-1} N_{i}\right)^{-1} \sum_{j_{0}=0}^{N_{0}-1} \ldots \sum_{j_{L-1}=0}^{N_{L-1}-1} g\left(z ; \sum_{i=0}^{L-1} \mu_{i, j_{i}}, \sum_{i=0}^{L-1} \sigma_{i}^{2}\right)
$$

From the previous expression, $f_{Z}(z)$ is a sum of $N_{Z}$ Gaussian functions, hence $f_{Z}(z)$ is a Gaussian mixture and can be expressed as

$$
\begin{gathered}
f_{Z}(z)=\mathrm{G}^{N_{Z}}\left(z, \mu_{Z}, \sigma_{Z}^{2}\right) \\
N_{Z}=\prod_{i=0}^{L-1} N_{i}, \sigma_{Z}^{2}=\sum_{i=0}^{L-1} \sigma_{i}^{2}
\end{gathered}
$$

$\mu_{Z}$ is a $1 \times N_{Z}$ vector that consists of the $N_{Z}$ summands in the expression

$$
\sum_{j_{0}=0}^{N_{0}-1} \ldots \sum_{j_{L-1}=0}^{N_{L-1}} \pi_{j_{0}, j_{1}, \ldots, j_{L-1}}
$$

where $\pi_{j_{0}, j_{1}, \ldots, j_{L-1}}=\sum_{i=0}^{L-1} \mu_{i, j_{i}}$.

Using Proposition 1 and (4.19), the conditional PDF of $\bar{s}_{l}$ given $s_{l}= \pm 1$ given $s_{l}= \pm 1$, is well approximated by

$$
f_{\bar{s}_{l} \mid s_{l}= \pm 1}(x) \approx \mathbf{G}^{N_{\bar{s}_{l}}}\left(x, \pm \boldsymbol{\mu}_{\bar{s}_{l}}, \sigma_{\bar{s}_{l}}^{2}\right)
$$

where $N_{\bar{s}_{l}}=\prod_{i=0}^{L-1} N_{i, k_{i}}, \sigma_{\bar{s}_{l}}^{2}=\sum_{i=0}^{L-1} d_{M_{i}}^{2}\left|\alpha_{i}\right|^{2} N_{0} / 2$, and $\mu_{\bar{s}_{l}}$ is a $1 \times N_{\bar{s}_{l}}$ vector that consists of the $N_{\bar{s}_{l}}$ summands in the expression $\sum_{j_{0}=0}^{N_{0}-1} \ldots \sum_{j_{L-1}=0}^{N_{L-1}} \pi_{j_{0}, j_{1}, \ldots, j_{L-1}}$ where $\pi_{j_{0}, j_{1}, \ldots, j_{L-1}}=$ $\sum_{i=0}^{L-1} \mu_{i, j_{i}}$ 


\subsubsection{Finding the instantaneous $B E R$}

The instantaneous BER given $\alpha_{0}, \alpha_{1}, \ldots, \alpha_{L-1}$ can be written as

$$
\begin{aligned}
B E R_{\text {inst }} & =\frac{1}{C} \sum_{l=0}^{C-1} p\left(\hat{s}_{l}=-1 \mid s_{l}=1\right) p\left(s_{l}=1\right)+p\left(\hat{s}_{l}=1 \mid s_{l}=-1\right) p\left(s_{l}=-1\right) \\
& =\frac{1}{C} \sum_{l=0}^{C-1} p\left(\bar{s}_{l}<0 \mid s_{l}=1\right)
\end{aligned}
$$

where we used the fact that $p\left(\hat{s}_{l}=-1 \mid s_{l}=1\right)=p\left(\hat{s}_{l}=1 \mid s_{l}=-1\right)$, because of the symmetry of $f_{\bar{s}_{l} \mid s_{l}= \pm 1}(x)$, and the equiprobable source assumption, i.e., $p\left(s_{l}=1\right)=$ $p\left(s_{l}=-1\right)=\frac{1}{2}$.

Using (4.22), $p\left(\bar{s}_{l}<0 \mid s_{l}=1\right)$ can be expressed as

$$
p\left(\bar{s}_{l}<0 \mid s_{l}=1\right)=\int_{-\infty}^{0} \mathrm{G}^{N_{\bar{s}_{l}}}\left(x, \boldsymbol{\mu}_{\bar{s}_{l}}, \sigma_{\bar{s}_{l}}^{2}\right)=\int_{-\infty}^{0} \frac{1}{N_{\bar{s}_{l}}} \sum_{i=0}^{N_{\bar{s}_{l}}-1} g\left(x, \mu_{\bar{s}_{l}, i}, \sigma_{\bar{s}_{l}}^{2}\right)=\frac{1}{N_{\bar{s}_{l}}} \sum_{i=0}^{N_{\bar{s}_{l}}-1} Q\left(\frac{\mu_{\bar{s}_{l}, i}}{\sigma_{\bar{s}_{l}}}\right) .
$$

Because the $Q$ function is a positive monotonic decreasing function, the previous expression can be bounded as follows

$$
\frac{1}{N_{\bar{s}_{l}}} Q\left(\frac{\min \left(\boldsymbol{\mu}_{\bar{s}_{l}}\right)}{\sigma_{\bar{s}_{l}}}\right)<p\left(\bar{s}_{l}<0 \mid s_{l}=1\right)<Q\left(\frac{\min \left(\boldsymbol{\mu}_{\bar{s}_{l}}\right)}{\sigma_{\bar{s}_{l}}}\right) .
$$

Since the $Q$ function dies out exponentially, the lower bound becomes very tight for high $\gamma_{i}$. Note that in the previous bound we only need to evaluate $\min \left(\boldsymbol{\mu}_{\bar{s}_{l}}\right)$, rather than evaluating all the elements of $\boldsymbol{\mu}_{\bar{s}_{l}}$.

It is not difficult to find that

$$
\min \left(\boldsymbol{\mu}_{\bar{s}_{l}}\right)=\sum_{i=0}^{L-1}\left|\alpha_{i}\right|^{2} d_{M_{i}}^{2} .
$$

Using the previous expression, the argument of the $Q$ function in (4.25) can be written as

$$
\min \left(\boldsymbol{\mu}_{\bar{s}_{l}}\right) / \sigma_{\bar{s}_{l}}=\frac{\sum_{i=0}^{L-1}\left|\alpha_{i}\right|^{2} d_{M_{i}}^{2}}{\sqrt{\sum_{i=0}^{L-1}\left|\alpha_{i}\right|^{2} d_{M_{i}}^{2} N_{0} / 2}}=\sqrt{\frac{\sum_{i=0}^{L-1}\left|\alpha_{i}\right|^{2} d_{M_{i}}^{2}}{N_{0} / 2}}=\sqrt{2 \sum_{i=0}^{L-1} d_{M_{i}}^{2} \gamma_{i}}=\sqrt{2 \gamma_{\text {out }}}
$$


where

$$
\gamma_{\text {out }}=\sum_{i=0}^{L-1} d_{M_{i}}^{2} \gamma_{i}
$$

This suggest that the output SNR of the SBMRC is a weighted sum of the individual SNRs, and these weights depend on the modulation levels of the signals to be combined. This again shows the similarities between conventional MRC and SBMRC. It is important to note that the argument of the $Q$ function is independent of the index $l$.

By substituting the previous expression in (4.25), the bounds simplify to

$$
\tau Q\left(\sqrt{2 \gamma_{\text {out }}}\right)<B E R_{\text {inst }}<Q\left(\sqrt{2 \gamma_{\text {out }}}\right)
$$

where $\tau$ is a constant that is related to the modulation levels of the signals to be combined and it is given by

$$
\tau=\frac{1}{C} \sum_{l=0}^{C-1} \frac{1}{N_{\bar{s}_{l}}}
$$

For example, $\tau=0.75$ for combining QPSK and 16-QAM, $\tau=0.5833$ for combining QPSK and 64-QAM and $\tau=0.5$ for combining 16-QAM and 64-QAM or for combining QPSK, 16-QAM and 64-QAM.

In Figs. $4.12,4.13$, and 4.14 we plot $B E R_{\text {inst }}$ using simulation and using the lower bound given by (4.29) for different instantaneous SNRs, for the scenarios $\left(M_{0}=4\right.$, $\left.M_{1}=16\right),\left(M_{0}=4, M_{1}=64\right)$, and $\left(M_{0}=16, M_{1}=64\right)$, respectively. In all cases, the analytical lower bound is very tight, especially for high SNRs.

From the bounds on the $B E R_{\text {inst }}$ given by (4.29), we can make the following observations:

1. Recall that $B E R_{\text {inst }}$ for classical MRC, where the signals to be combined have the same modulation level $M$, is proportional to $Q\left(\sqrt{2 d_{M}^{2} \sum_{i=0}^{L-1} \gamma_{i}}\right)[23]$, which 
means that both the SBMRC and MRC achieve the same output SNR when the modulation levels are the same.

2. Let $\gamma_{(i)}$ denotes the $i^{\text {th }}$ smallest $\gamma_{i}$, that is, $\gamma_{(0)} \leq \gamma_{(1)} \leq \ldots \leq \gamma_{(L-1)}$. If $\left\{\gamma_{(i)}\right\}_{i=0}^{L-1}$ are known at the BS, then, to maximize the output SNR at UT (refer to (4.28)), the modulation levels should be assigned such that $d_{M_{(0)}} \leq$ $d_{M_{(1)}} \leq \ldots \leq d_{M_{(L-1)}}$, which means that $M_{(0)} \geq M_{(1)} \geq \ldots \geq M_{(L-1)}$. In other words, lower modulation levels should be used in the links that experience better channel conditions, and vice versa. Note that for a set of modulation levels $\left\{M_{(i)}\right\}_{i=0}^{L-1}$, the end-to-end spectral efficiency is the same regardless of the assignments of these modulation levels to the transmitting nodes. Hence, using this rule will not only maximize the output SNR but also maximizes the throughput. Remark that this rule is reversed in conventional AMC without diversity combining. 


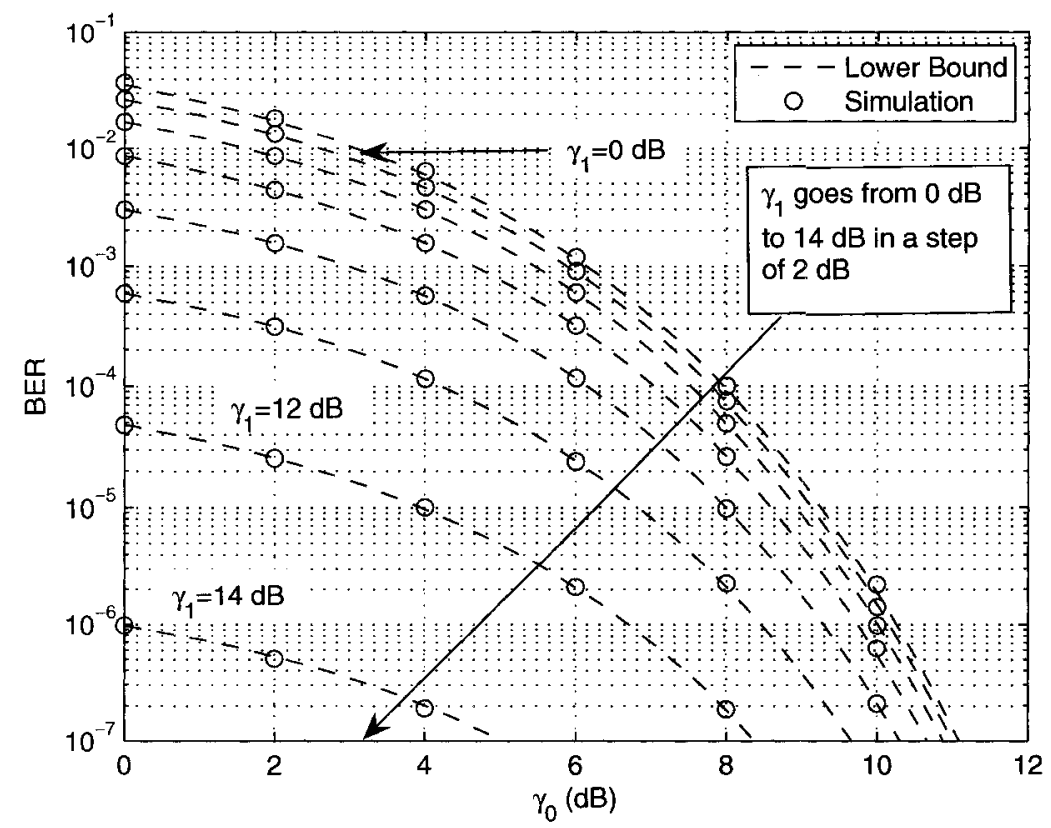

Figure 4.12: Instantaneous BER performance of SBMRC for $\left(M_{0}=4, M_{1}=16\right)$.

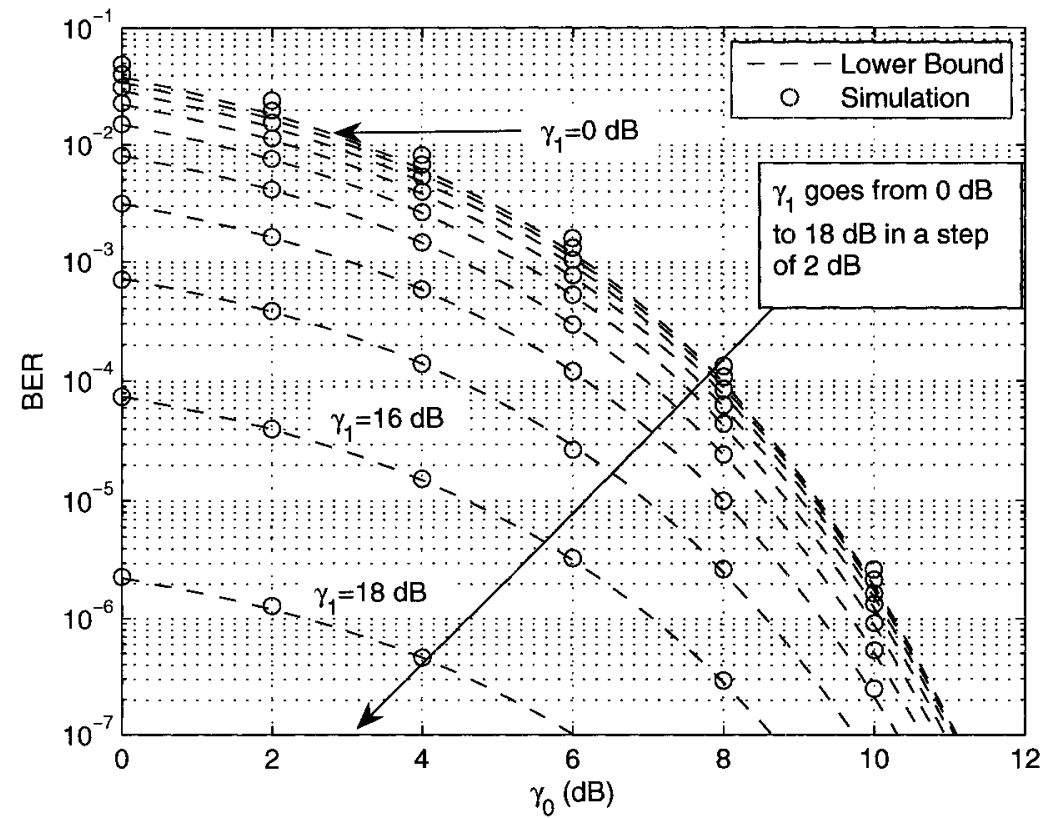

Figure 4.13: Instantaneous BER performance of SBMRC for $\left(M_{0}=4, M_{1}=64\right)$. 


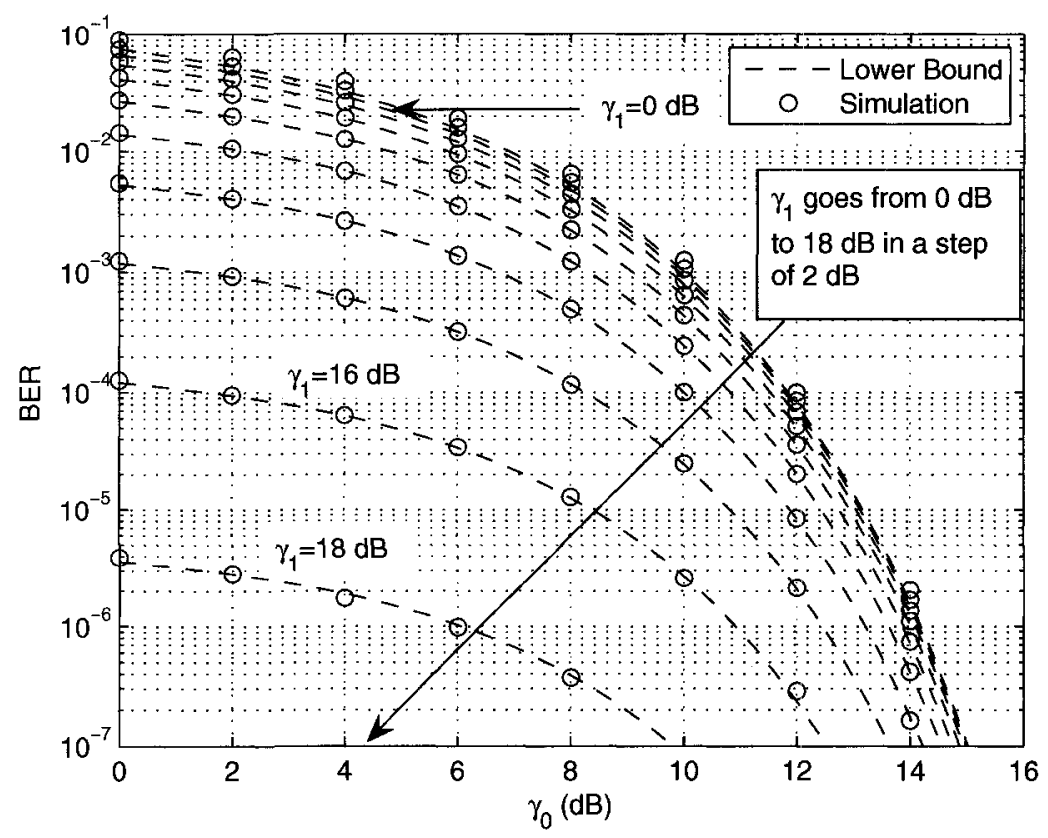

Figure 4.14: Instantaneous BER performance of SBMRC for $\left(M_{0}=16, M_{1}=64\right)$.

\subsubsection{Finding the average $B E R$}

Equation (4.29) gives bounds on the instantaneous BER for a given $\gamma_{\text {out }}$. To get bounds on the average BER, the expression in (4.29) is averaged over the PDF of $\gamma_{\text {out }}$. Because of the similarity between the output SNR expression for SBMRC and for classical MRC, the same procedure for finding the average BER (when MRC is employed) can be used. The details of this procedure are given in [23], and only the final result is shown here. The average BER can be bounded as

$$
\frac{1}{2} \tau \sum_{i=0}^{L-1} \pi_{i}\left(1-\sqrt{\frac{d_{M_{i}}^{2} \bar{\gamma}_{i}}{1+d_{M_{i}}^{2} \bar{\gamma}_{i}}}\right)<B E R<\frac{1}{2} \sum_{i=0}^{L-1} \pi_{i}\left(1-\sqrt{\frac{d_{M_{i}}^{2} \bar{\gamma}_{i}}{1+d_{M_{i}}^{2} \bar{\gamma}_{i}}}\right)
$$

where $\pi_{i}=\prod_{j=0, j \neq i}^{L-1} \frac{d_{M_{i}}^{2} \bar{\gamma}_{i}}{d_{M_{i}}^{2} \bar{\gamma}_{i}-d_{M_{j}}^{2} \bar{\gamma}_{j}}$.

These bounds can be approximated for $\gamma_{i} \gg 1$ as 


$$
\begin{gathered}
\tau\left(\begin{array}{c}
2 L-1 \\
L
\end{array}\right) \prod_{i=0}^{L-1} \frac{1}{4 d_{M_{i}}^{2} \bar{\gamma}_{i}}<B E R<\left(\begin{array}{c}
2 L-1 \\
L
\end{array}\right) \prod_{i=0}^{L-1} \frac{1}{4 d_{M_{i}}^{2} \bar{\gamma}_{i}} \\
\Rightarrow \tau\left(\begin{array}{c}
2 L-1 \\
L
\end{array}\right)\left(\prod_{i=0}^{L-1} \frac{1}{4 d_{M_{i}}^{2} \sigma_{i}^{2}}\right) \mathbf{S N R}^{-L}<B E R<\left(\begin{array}{c}
2 L-1 \\
L
\end{array}\right)\left(\prod_{i=0}^{L-1} \frac{1}{4 d_{M_{i}}^{2} \sigma_{i}^{2}}\right) \mathbf{S N R}^{-L}
\end{gathered}
$$

The upper bound in the above gives an explicit proof that the SBMRC achieves a diversity order of $L$, i.e., full diversity. As stated earlier, the lower bound is very tight for high SNR and can be used as a very good approximation of BER. From the previous bounds, since the multiplication operation is commutative, we conclude that asymptotically, the average BER is independent of the order of the modulation schemes to be combined. That is, assigning lower modulation levels in the links that experience better average channel qualities does not improve the BER asymptotically, in contrast to the case of the instantaneous BER derived in (4.29).

\subsection{Analytical and Simulation Results}

In this section, we present simulation and analytical results of the BER performance of SC, BSC, MLD, SBMLD, and SBMRC ${ }^{4}$.

To compare the performance of SC, BSC, SBMLD, SBMRC, and MLD, we consider relay networks with $L=2$ (single relay) and $L=3$ (two relays). For the sake of presentation, we assume the average channel conditions to be the same in all the links, i.e., $\bar{\gamma}_{i}=\bar{\gamma}$ for $i \in\{0,1, \ldots, L-1\}$. Table 4.2 shows the loss in SNR of all schemes compared to the optimum MLD scheme for different scenarios. The BSC always outperforms $\mathrm{SC}$ which makes it a better selection combining scheme as dis-

\footnotetext{
${ }^{4}$ See appendix A for the detailed confidence interval calculation.
} 
cussed in Chapter 3. The SC suffers from higher performance loss as the difference between the modulation levels increases, such as in scenarios 2 and 5 . This is due to the fact that SC relies solely on $\gamma_{i}$ and ignores the difference in reliabilities incurred by different modulation levels. On the contrary, BSC accounts for both through the calculation of BER and selecting the branch that will result in the least number of errors(on average). This explains why BSC does not suffer such a high performance loss in scenarios 2 and 5. Although BSC rectifies some of the drawbacks of SC, it is still far from the optimal MLD by 1.62 to $3.12 \mathrm{~dB}$, depending on the scenario. Both SBMLD and SBMRC have very close performance to the MLD scheme (degradation is less than $0.3 \mathrm{~dB}$ ). Note that the loss in SNR is measured at a BER of $10^{-3}$, which is a reasonable value for uncoded schemes. Nevertheless, it is observed that the loss in SNR for both SBMLD and SBMRC vanishes at very low BER. The SBMLD provides negligible performance gain compared to the SBMRC at the expense of the computation of the conditional PDFs of the soft bits. For these reasons, SBMRC is the most attractive scheme from the practical point of view; it is very simple to implement, with negligible performance degradation compared to the MLD and SBMLD schemes.

Figures 4.15 and 4.16 show the simulation results for the BER performance of SBMRC for combining signals with different modulation levels, for one and two relays, respectively. The lower bound given by (4.31) is evaluated for each scenario and plotted in the same figure. It is clear that the lower bound is very tight for all scenarios and it can be used as a very good approximation for evaluating the BER for SBMRC. This again validates the accuracy of approximating the conditional PDF by a Gaussian mixture.

It is also clear that all curves in Fig. 4.15 decay two orders of magnitude per 
decade (diversity order of 2) and all curves in Fig. 4.16 decay three orders of magnitude per decade (diversity order of 3). Consequently, SBMRC achieves full diversity (diversity order of $L$ ), as proven by (4.32). Note that the results for the case where $M_{i}=2($ BPSK) were not shown since both BPSK and QPSK have the same BER performance.

In Fig. 4.17, we compare the BER performance of SBMRC with SC and BSC, in single-relay and two-relay networks with $\left(M_{0}=4, M_{1}=64\right)$, and $\left(M_{0}=4, M_{1}=\right.$ $16, M_{2}=64$ ), respectively ${ }^{5}$. We observe that all the three schemes achieve the same diversity order. However, SBMRC achieves a higher SNR gain (or coding gain) represented by a horizontal shift to the left of the BER curve compared to both SC and BSC. SBMRC has an SNR gain of about $1.6 \mathrm{~dB}$ and $3 \mathrm{~dB}$, over BSC, in the first and second scenarios, respectively. Moreover, SBMRC has an SNR gain of about 3.8 $\mathrm{dB}$ and $5.2 \mathrm{~dB}$ over $\mathrm{SC}$ in the first and second scenarios, respectively. Again, BSC outperforms SC by about $2.2 \mathrm{~dB}$ in both scenarios for the same reasons mentioned before.

Even though we showed only the results when the average channel conditions are the same, the same conclusions were observed when the average channel conditions are different.

From this section we conclude that SBMRC is the most suitable receiver structure in terms of BER performance and complexity.

\footnotetext{
${ }^{5}$ The BER curves for both MLD and SBMLD are not shown in the figure since the difference between their BER performance and SBMRC is minute and not noticeable in the figure. Thus, we refer the reader to Table 4.2 for accurate comparison between SBMRC, SBMLD, and MLD.
} 
Table 4.2: Loss in SNR (dB) at BER $=10^{-3}$ of SC, BSC, SBMLD and SBMRC compared to the optimum MLD.

\begin{tabular}{|c|c|c|c|c|}
\hline Scheme & SC & BSC & SBMLD & SBMRC \\
\hline$M_{0}=4, M_{l}=16$ & & & & \\
\hline$M_{0}=4, M_{l}=64$ & 2.30 & 1.62 & 0.00 & 0.02 \\
\hline$M_{0}=16, M_{1}=64$ & 4.10 & 1.94 & 0.06 & 0.09 \\
\hline$M_{0}=4, M_{1}=4, M_{2}=16$ & 3.73 & 1.95 & 0.06 & 0.08 \\
\hline$M_{0}=4, M_{1}=4, M_{2}=64$ & 6.48 & 3.10 & 0.22 & 0.07 \\
\hline$M_{0}=4, M_{1}=16, M_{2}=16$ & 3.36 & 2.71 & 0.09 & 0.09 \\
\hline$M_{0}=16, M_{I}=16, M_{2}=64$ & 3.93 & 3.12 & 0.12 & 0.13 \\
\hline$M_{0}=16, M_{I}=64, M_{2}=64$ & 3.63 & 2.95 & 0.09 & 0.09 \\
\hline
\end{tabular}

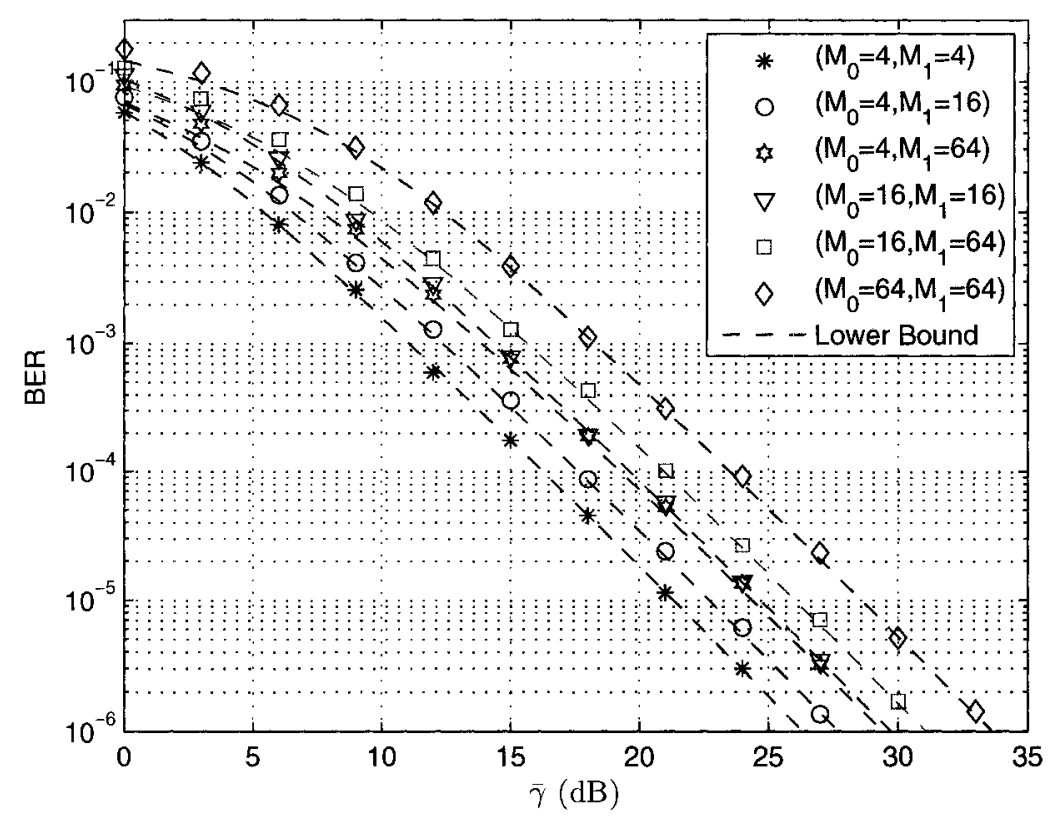

Figure 4.15: BER performance of diversity combining using SBMRC for $L=2$ 


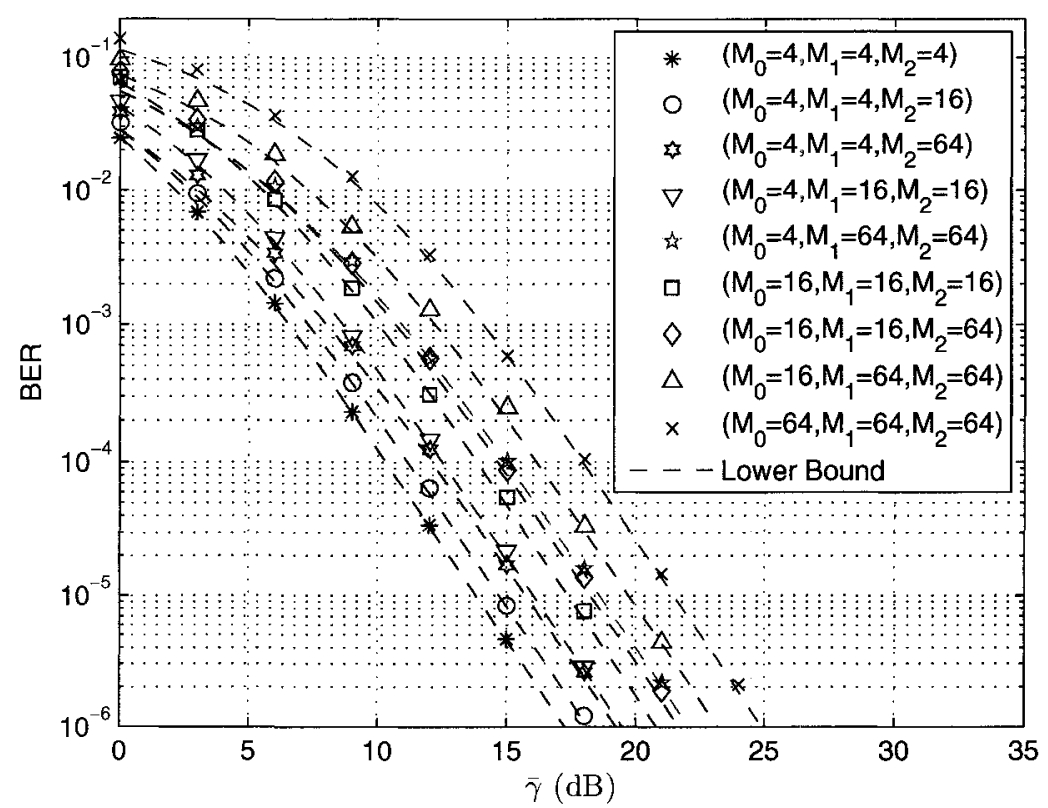

Figure 4.16: BER performance of diversity combining using SBMRC for $L=3$

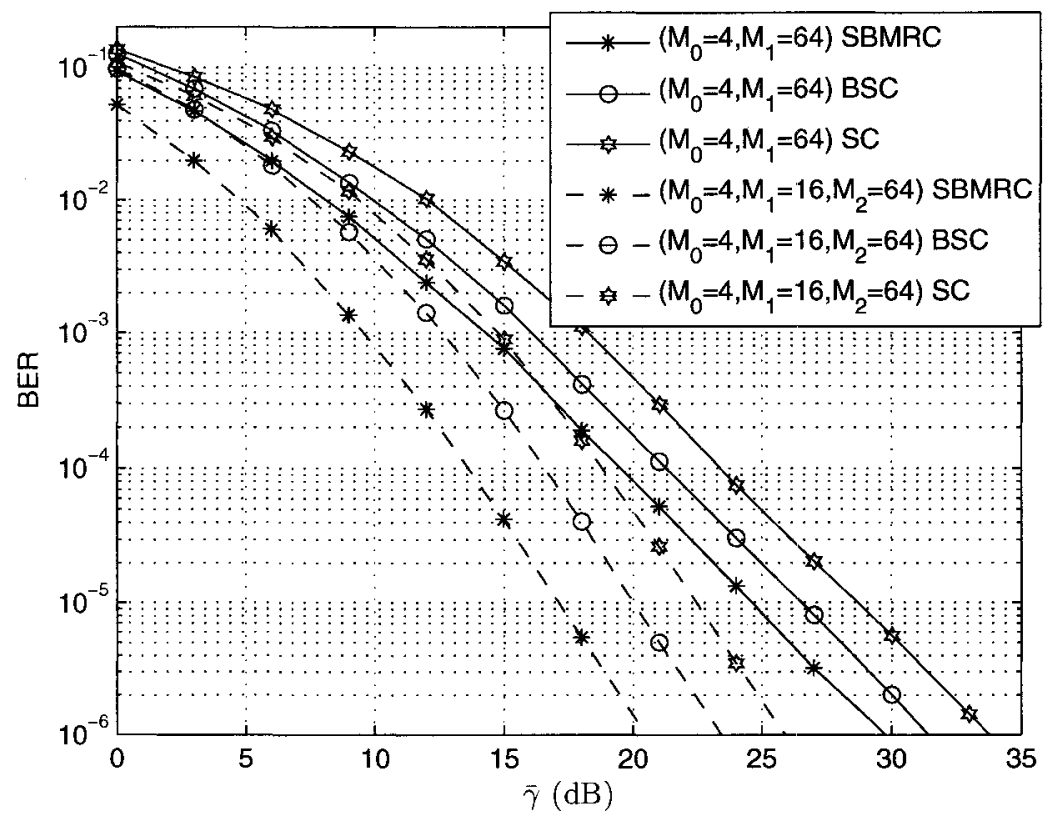

Figure 4.17: BER performance of SC, BSC, and SBMRC. 


\subsection{Adaptive Modulation and Diversity Combining}

In the previous sections we found that SBMRC is the most suitable receiver from both performance and complexity perspectives. In this section, we show the gain obtained in the spectral efficiency by employing SBMRC.

Similar to [10], we assume assigning the modulation levels for both the BS and RS are done at the BS and that the UT is communicating with the BS either directly or with the help of a single RS. For the direct transmission case, where communication happens without an RS, the end-to-end spectral efficiency $\delta$ is simply $K_{0}=\log _{2}\left(M_{0}\right)$. However, when cooperation through an RS is involved, $\delta$ is given by [10] [11]

$$
\delta=\left(\left(\log _{2}\left(M_{0}\right)\right)^{-1}+\left(\log _{2}\left(M_{1}\right)\right)^{-1}\right)^{-1}=\left(K_{0}^{-1}+K_{1}^{-1}\right)^{-1} .
$$

We define two modes of operations, namely dynamic and static. In dynamic (static) adaptive modulations, the BS knows the instantaneous (average) channel conditions $\gamma_{0}$ and $\gamma_{1}\left(\bar{\gamma}_{0}\right.$ and $\left.\bar{\gamma}_{1}\right)$ and it assigns the modulations for both BS and RS such that the end-to-end spectral efficiency is maximized while keeping the instantaneous (average) BER below the targeted BER which is denoted by $P_{t}$. The dynamic mode of operation is applicable to low mobility users where the channel changes slowly and obtaining CSI at BS is feasible. On the other hand, the static mode of operation is applicable to high mobility users where the channel changes rapidly and only the average CSI is available at BS. We restrict the modulation schemes to be used to QPSK, 16-QAM, and 64-QAM and we do not employ channel coding.

\subsubsection{Dynamic adaptive modulation}

Table 4.3 summarizes all the possible modes to be used and it shows the spectral efficiency of each mode. It also shows the condition on the pair of SNRs $\left(\gamma_{0}, \gamma_{1}\right)$ 
where the mode can be used such that it achieves the required instantaneous BER, $P_{t}$. If more than one mode can be used, then the BS chooses the mode that achieves the maximum spectral efficiency. The instantaneous BER was obtained by evaluating the tight lower bound given in (4.29) for different modulation schemes. The shaded modes are the modes that requires the SBMRC, while the other modes can be used using conventional MRC. In Fig. 4.18, we apply the conditions on Table 4.3 to find the SNR region for each mode for $P_{t}=10^{-3}$. This figure can be used at the BS as a two dimensional look-up table to decide the best mode for a given SNR pair $\left(\gamma_{0}, \gamma_{1}\right)$. For example, if $\left(\gamma_{0}=9 d B, \gamma_{1}=10 d B\right)$, then the BS will transmit using 64-QAM and the RS will transmit using 16-QAM. The shaded areas represent the regions where SBMRC is used and a gain in spectral efficiency is observed. The gains in these regions achieved by using SBMRC in combining (QPSK, 16-QAM), (QPSK, 64-QAM), and (16-QAM, 64-QAM) are 33\%, 50\%, and $20 \%$, respectively. The gain in spectral efficiency is measured with respect to the mode with the highest spectral efficiency that doesn't use SBMRC and achieves BER less than or equal to $P_{t}$. For example, in the region where the mode is (64-QAM, QPSK, $\delta=1.5)$, the gain is measured with respect to the mode (QPSK, QPSK, $\delta=1$ ), (as this is the mode will be used without SBMRC ), which means a gain of $50 \%$ in spectral efficiency. The figure also shows the regions where direct transmission is better than cooperation. 
Table 4.3: The modes for the dynamic mode of operation.

\begin{tabular}{|c|c|c|c|}
\hline Mode & Instantaneous BER & $\begin{array}{c}\text { Condition on } \\
\gamma_{0} \text { and } \gamma_{1}\end{array}$ & $\begin{array}{l}\text { Spectral efficiency } \\
(\delta)\end{array}$ \\
\hline $\mathrm{M}_{0}=4$ (Direct Transmission) & $Q\left(\sqrt{2 \gamma_{0}}\right)$ & $\gamma_{0} \geq 0.5\left(Q^{-1}\left(P_{t}\right)\right)^{2}$ & 2 \\
\hline $\mathrm{M}_{0}=16$ (Direct Transmission) & $0.75 Q\left(\sqrt{0.8 \gamma_{0}}\right)$ & $\gamma_{0} \geq 1.25\left(Q^{-1}\left(1.33 P_{r}\right)\right)^{2}$ & 4 \\
\hline $\mathrm{M}_{0}=64$ (Direct Transmission) & $0.58 Q\left(\sqrt{0.29 \gamma_{0}}\right)$ & $\gamma_{0} \geq 3.45\left(Q^{-1}\left(1.72 P_{t}\right)\right)^{2}$ & 6 \\
\hline MRC, $\left(M_{0}=4, M_{1}=4\right)$ & $Q\left(\sqrt{2\left(\gamma_{0}+\gamma_{1}\right)}\right)$ & $\gamma_{0}+\gamma_{1} \geq 0.5\left(Q^{-1}\left(P_{t}\right)\right)^{2}$ & 1 \\
\hline ( & $0.75 Q(\sqrt{2 \gamma+0.87)}$ & $2 y+0.8 \eta x \geq(0,(133 p))^{3}$ & 1.33 \\
\hline sBMre, $\left(M_{i}=16, M_{i}=4\right.$ & $0.0 .750(\sqrt{0.8 \gamma(y+2 \gamma)}$ & 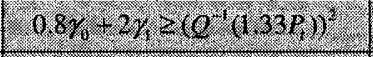 & \\
\hline 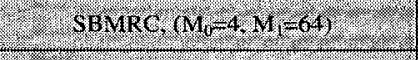 & $-\left(0.580\left(\sqrt{2} \gamma_{0}+0.29 \gamma_{1}\right)\right.$ & 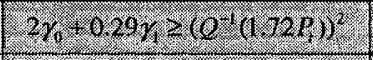 & \\
\hline S SBMRC: $\left(M_{0}=64, M_{1}-4\right)$ & $8 .(1580(\sqrt{0.29})(2 y)$ & $\left(0.297+2 y \geq\left(g^{-1}(1.72 p)\right)^{2}\right.$ & \\
\hline Without combining, $\left(M_{0}=64, M_{1}=4\right)$ & $Q\left(\sqrt{2 \gamma_{1}}\right)$ & $\gamma_{1} \geq 0.5\left(Q^{-1}\left(P_{t}\right)\right)^{2}$ & 1.5 \\
\hline $\mathrm{MRC},\left(\mathrm{M}_{0}=16, \mathrm{M}_{1}=16\right)$ & $0.75 Q\left(\sqrt{0.8\left(\gamma_{0}+\gamma_{1}\right)}\right)$ & $\gamma_{0}+\gamma_{1} \geq 1.25\left(Q^{-1}\left(1.33 P_{t}\right)\right)^{2}$ & 2 \\
\hline 2. spMrc, $(M-16 . \mathrm{M}=64)$ & $0.50\left(\sqrt{\left.0.8 \gamma_{1}+0.29 \gamma_{1}\right)}\right.$ & 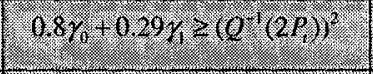 & 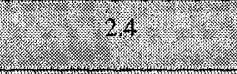 \\
\hline SBMRC, $\left(\mathrm{M}_{1}=64, \mathrm{M}_{1}=16\right)$ & $(0.50(\sqrt{0.29 y+0.8 y})$ & $0.297+0.8 \%=\left(0^{-1}(2 p)\right)^{2}$ & 2.28 \\
\hline Without combining, $\left(\mathrm{M}_{0}=64, \mathrm{M}_{1}=16\right)$ & $0.75 Q\left(\sqrt{0.8 \gamma_{1}}\right)$ & $\gamma_{1} \geq 1.25\left(Q^{-1}\left(1.33 P_{t}\right)\right)^{2}$ & 2.4 \\
\hline MRC, $\left(M_{0}=64, M_{l}=64\right)$ & $0.58 Q\left(\sqrt{0.29\left(\gamma_{0}+\gamma_{1}\right)}\right)$ & $\gamma_{0}+\gamma_{1} \geq 3.45\left(Q^{-1}\left(1.72 P_{t}\right)\right)^{2}$ & 3 \\
\hline
\end{tabular}




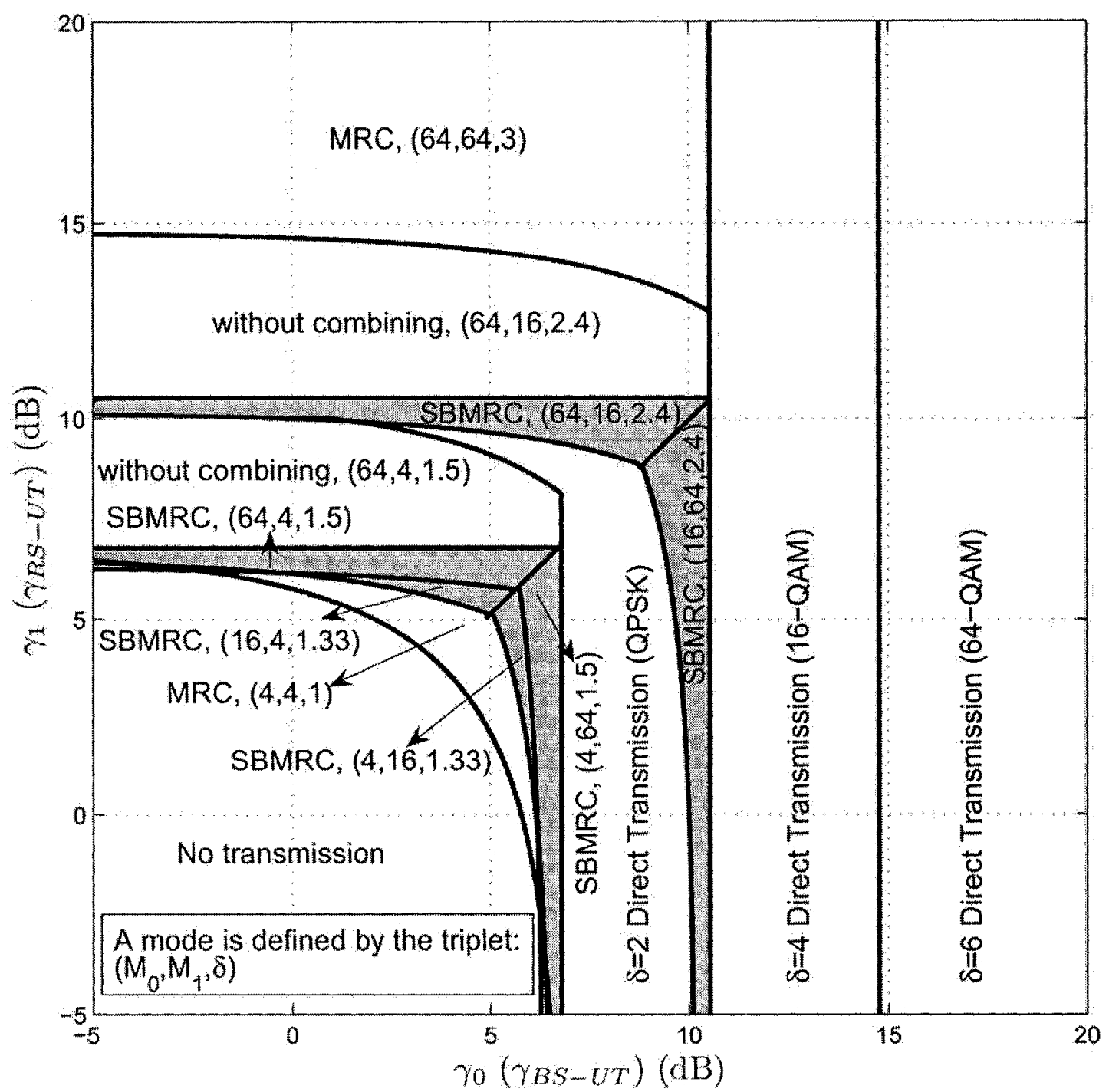

Figure 4.18: The SNR regions for each mode in the dynamic adaptive modulation.

\subsubsection{Static adaptive modulation}

Table 4.4 summarizes all the possible modes to be used and it shows the spectral efficiency of each mode. If more than one mode can be used, then the BS chooses the mode that achieves the maximum spectral efficiency. The average BER was obtained by evaluating the tight lower bound given in (4.31) for different modulation schemes. 
The shaded modes are the modes that requires SBMRC, while the other modes can be used using conventional MRC. Finding the pair of average SNRs $\left(\bar{\gamma}_{0}, \bar{\gamma}_{1}\right)$ where the mode can be used such that it achieves the required average BER, $P_{t}$, is difficult. Thus, in Fig. 4.18 we numerically invert the average BER expression to find the SNR region for each mode such that it achieves an average BER that is less than or equal to $P_{t}=10^{-3}$. This figure can be used at the BS as a two dimensional look-up table to decide the best mode for a given average SNR pair $\left(\bar{\gamma}_{0}, \bar{\gamma}_{1}\right)$. For example, if $\left(\bar{\gamma}_{0}=5 d B, \bar{\gamma}_{1}=20 d B\right)$, then the BS will transmit using 64-QAM and the RS will transmit using QPSK. The shaded areas represent the regions where SBMRC is used and a gain in spectral efficiency is observed. The gains in these regions achieved by using SBMRC in combining (QPSK, 16-QAM), (QPSK, 64-QAM), and (16-QAM, $64-\mathrm{QAM})$ are $33 \%, 50 \%$, and $20 \%$, respectively. The gain in spectral efficiency is measured with respect to the mode with the highest spectral efficiency that doesn't use SBMRC and achieves BER less than or equal to $P_{t}$. For example, in the region where the mode is (16-QAM, QPSK, $\delta=1.33$ ), the gain is measured with respect to the mode (QPSK, QPSK, $\delta=1$ ), (as this is the mode will be used without SBMRC ), which means a gain of $33 \%$ in spectral efficiency. The figure also shows the regions where direct transmission is better than cooperation. 
Table 4.4: The modes for the static mode of operation.

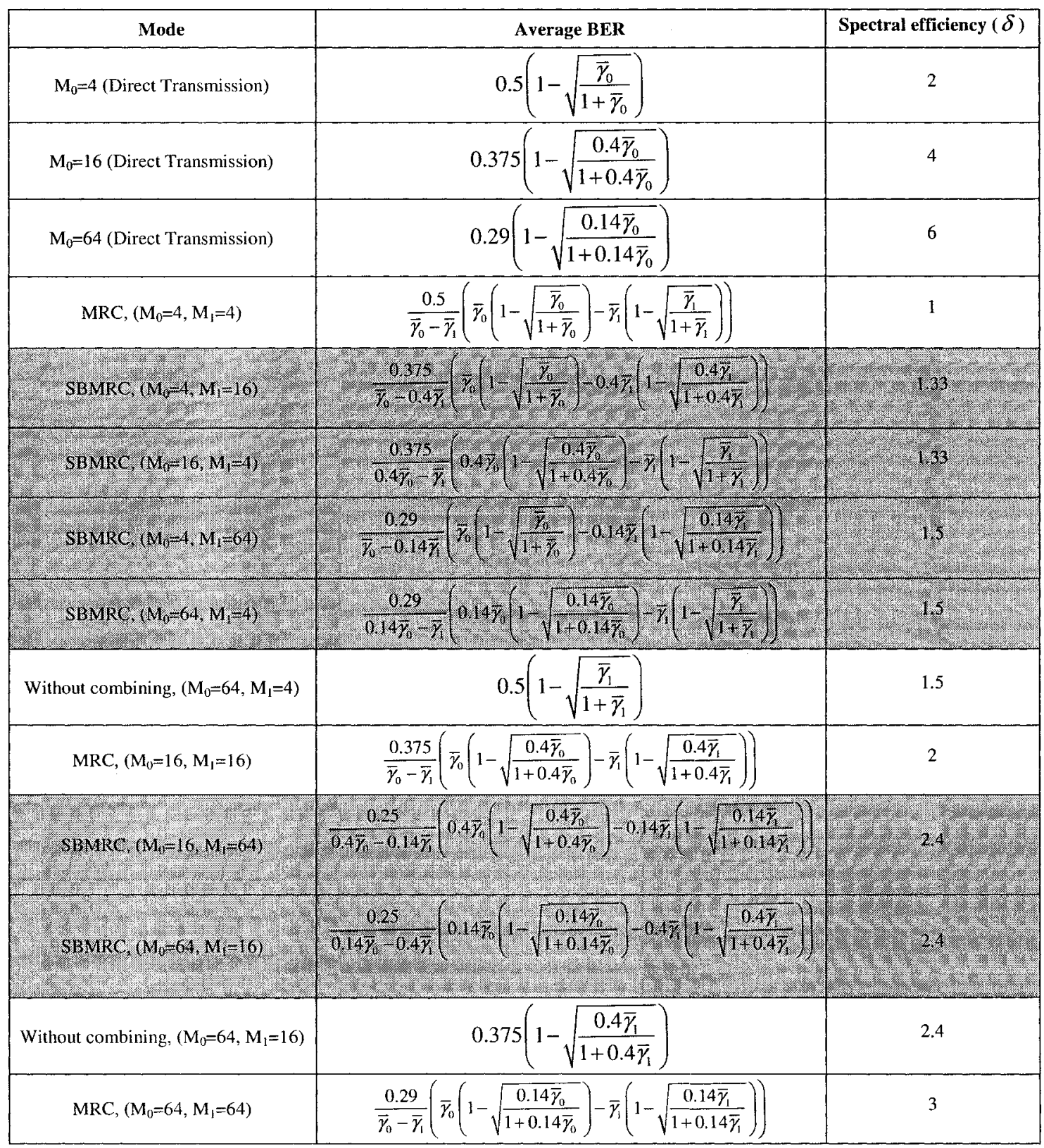




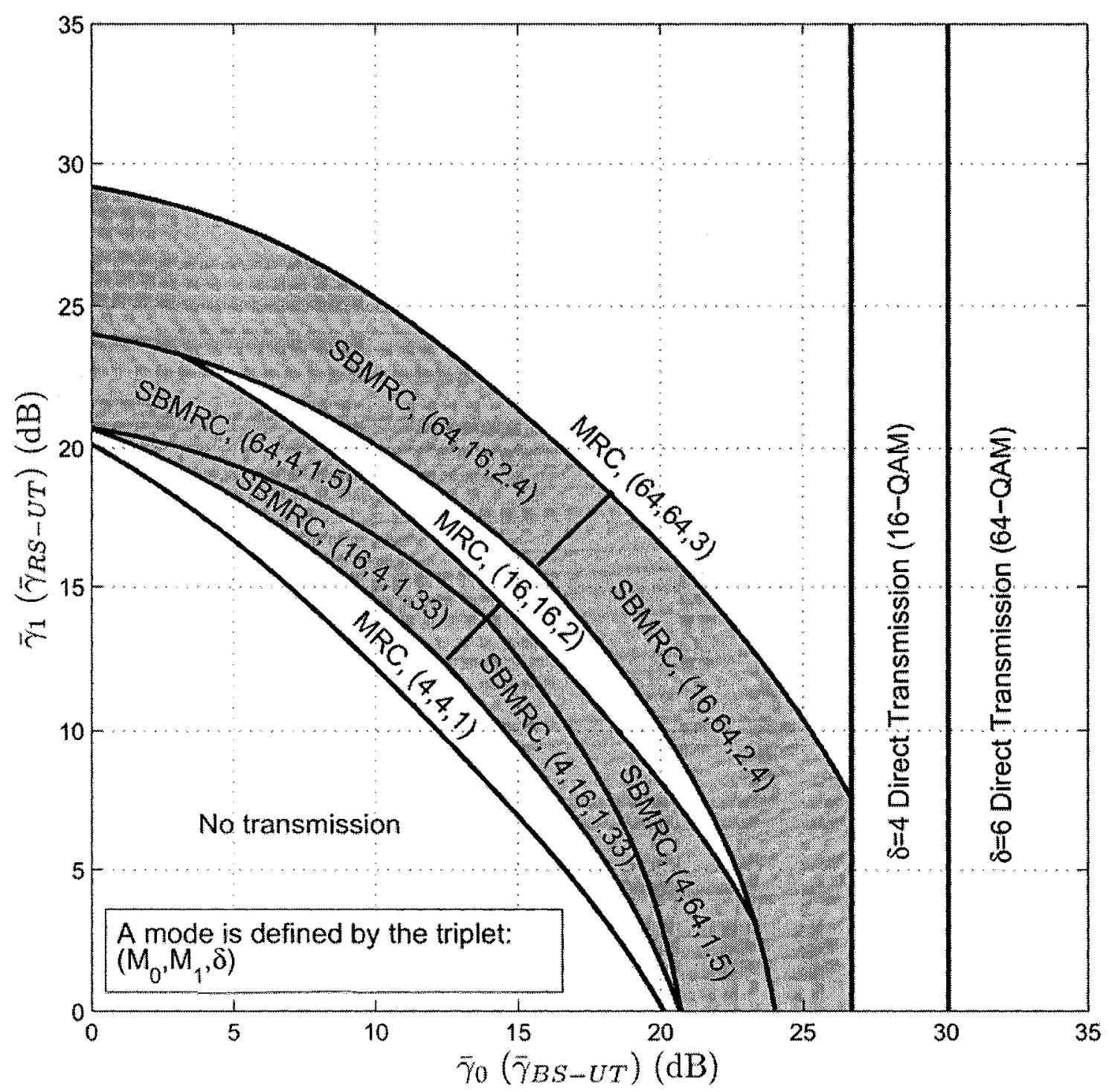

Figure 4.19: The SNR regions for each mode in the static adaptive modulation. 


\section{Chapter 5}

\section{Diversity Combining of Signals with Different Modulation Levels in Nomadic Relay Networks}

In the previous chapters, we investigated different receiver structures and we concluded that SBMRC is the most attractive structure for combining signals with different modulations in fixed relay networks. In this chapter, we investigate how to use SBMRC in nomadic relay networks where the source-relay links are not error-free. In Section 5.1, we review the existing techniques proposed in literature to mitigate error propagation when the same modulation scheme is used for all transmitting nodes. Then in Section 5.2, we explain how to apply one of these techniques if the transmitting nodes do not use the same modulation. Moreover, we propose a better error-mitigation scheme in Section 5.3. In addition, BER performance analysis is presented in Section 5.4 and followed by analytical and simulation results in Section 5.5 .

\subsection{Existing techniques to mitigate error propagations}

As stated in [24], for a single relay network, if simple decode-and-forward (DF) relaying (the relay always forward the received signal from the BS) is employed and MRC is performed at the UT, significant performance lose is observed as the diversity order is reduced from 2 to 1 due to error propagation. We call this scheme simple 
DF. A trivial solution to the error propagation problem is to employ error detection codes (such as CRC), and the message at the relay is detected and checked for errors with the help of these codes. If an error is detected, then the relay does not forward the erroneously decoded signals. Such a method will perform very well in terms of mitigating error propagation. However, performing error detection results in additional delay and adds to the complexity at the RS [24]. This fact motivated different researchers to find more efficient error-mitigation schemes [19, 24,33-40].

In [39], the receiver structure is modified to account for error propagation. The relay always transmits, and the receiver is designed as a MLD assuming it has the average BER in the source to relay links. The authors also simplify the MLD into a piecewise linear receiver. It is shown that such a receiver achieves a diversity order of $n$ where $(L+1) / 2 \leq n \leq L / 2+1$ if $L$ is even and $n=(L+1) / 2$ if $L$ is odd. In [40], a better receiver structure that is called Cooperative-MRC (C-MRC) is proposed. The proposed receiver achieves full diversity at the expense of obtaining the instantaneous BER of source-relay links at the UT. Both the proposed schemes in [39] and [40] require additional signalling to estimate the BER of source-relay links and make them available at the UT. This requirement is the main drawback of these schemes. Such drawback has motivated the research on SNR-based threshold relaying $[33-36]$.

In SNR-based threshold relaying, the RS makes the decision whether to transmit or not by comparing the instantaneous SNR of the source-relay link to a predefined threshold. This scheme is also referred to as on-off relaying. Unlike [39] and [40], the receiver at UT is simple MRC. In [5] and [36], it is assumed that the sourcerelay link is error free (through advanced channel coding), as long as the SNR in the source-relay link is greater than a target SNR, which is the threshold to be used 
at the RS. In [33], the problem of finding the threshold to minimize the end-to-end BER is formulated as joint threshold selection and power allocation problem, and it is solved numerically. The power allocation problem is defined as how much power should be allocated to the RS such that the total transmitted power (power of the source plus the power of the RS) is fixed. In [35], a similar problem to the one in [33] is tackled with the assumption that the channel coefficients are real Gaussian random variables, which does not apply to practical wireless channel. In [34], it is concluded that threshold relaying is beneficial in multi-antenna relays and the choice of the threshold becomes less critical as the number of antennas at the relays increase. More recently, the problem of threshold selection is studied rigourously in [24] and analytical expression of the optimal threshold is derived for different assumptions of the availability of the instantaneous and average SNR information at the RS, assuming BPSK modulation. It is concluded that the knowledge of the instantaneous source-destination SNR improves the performance only in the high SNR region, while the knowledge of the instantaneous relay-destination SNR provides negligible gain in performance. In [38], it is proven analytically that threshold-relaying achieves full diversity for single RS given that the threshold is chosen properly, such as the threshold derived in [24].

Instead of having on-off relaying, the RS can continuously scale its transmit power depending on SNR of the source-relay and the relay-destination links, as proposed in $[19,37]$. This scheme is called LAR and can be viewed as a generalized form of the on-off relaying. It is shown that this scheme achieves full diversity and requires simple channel state information that can be easily made available at the RS. The LAR exhibits robustness to quantization and feedback errors in estimating the channel conditions in the relay-to-destination link. The LAR is universally applicable to any 
modulation.

It is worth repeating that the same modulation scheme is assumed for both source and relay in all the aforementioned papers.

For our purpose, we implement LAR because it is applicable to any modulation scheme and it requires only the average relay-destination SNR, which can be easily fed back from the UT $[19,37]$. We show in the subsequent section that SBMRC can also achieve full diversity when LAR is implemented for error propagation mitigation. Moreover, we propose a modified version of the LAR that can further improve the SNR gain.

\subsection{Link Adaptive Regeneration}

The LAR strategy proposed in [19] assumes that the relay can scale its transmission power by $\kappa_{i}$ such that $\kappa_{i} \in[0,1]$. The coefficient $\kappa_{i}$ for $\mathrm{RS}_{i}$ is defined as

$$
\kappa_{i}:=\frac{\min \left(\gamma_{B S-R S_{i}}, \gamma_{R S_{i}-U T}\right)}{\gamma_{R S_{i}-U T}}=\left\{\begin{array}{c}
\frac{\gamma_{B S^{-}-R S_{i}}}{\bar{\gamma}_{R S_{i}-U T}} \text { if } \gamma_{B S-R S_{i}}<\gamma_{R S_{i}-U T} \\
1 \text { if } \gamma_{B S-R S_{i}} \geq \gamma_{R S_{i}-U T}
\end{array}\right.
$$

or as

$$
\kappa_{i}:=\frac{\min \left(\gamma_{B S-R S_{i}}, \bar{\gamma}_{R S_{i}-U T}\right)}{\bar{\gamma}_{R S_{i}-U T}}=\left\{\begin{array}{c}
\frac{\gamma_{B S-R S_{i}}}{\bar{\gamma}_{R S_{i}-U T}} \text { if } \gamma_{B S-R S_{i}}<\bar{\gamma}_{R S_{i}-U T} \\
1 \text { if } \gamma_{B S-R S_{i}} \geq \bar{\gamma}_{R S_{i}-U T}
\end{array}\right.
$$

Although better performance can be achieved by using (5.1) instead of (5.2), in this research we use (5.2). This is because knowing $\gamma_{R S_{i}-U T}$ accurately at the relay is significantly harder than $\bar{\gamma}_{R S_{i}-U T}$, especially for rapidly varying channel. The rational behind defining $\kappa_{i}$ in such a manner is to transmit with maximum power if the instantaneous SNR in the BS-RS $S_{i}$ link is larger than the average SNR in the $\mathrm{RS}_{i}$-UT link, which indicates that the decoded symbol at the $\mathrm{RS}_{i}$ is reliable; otherwise, the $\mathrm{RS}_{i}$ will transmit with only a fraction of the maximum power because that is an in- 
dication of unreliable decoding. With such a strategy, the effect of error-propagation is significantly reduced. By using LAR, the effective instantaneous SNR in the link from $\mathrm{RS}_{i}$ to UT seen by the UT is $\kappa_{i} \gamma_{R S_{i}-U T}$. Estimating $\gamma_{B S-R S_{i}}$ can be done using training symbols and it comes at no additional cost, since estimating the channel coefficient is already needed for coherently decoding the M-QAM signal received from BS. Estimating $\bar{\gamma}_{R S_{i}-U T}$ is done at the UT and it is fed back to the $\mathrm{RS}_{i}$. We neglect the quantization error introduced by representing $\bar{\gamma}_{R S_{i}-U T}$ with a finite number of bits. Nevertheless, LAR is shown in [19] to be robust to quantization errors. In [19], MRC is used at the UT and the same modulation is assumed for all transmitting nodes. The MRC uses the equivalent channel coefficient in the link $\mathrm{RS}_{i}$-UT which is equal to $\sqrt{\kappa_{i}} \alpha_{i}$. In this work, the UT uses SBMRC, and the BS and $\mathrm{RS}_{i}$ use different modulation schemes.

\subsection{Modified Link Adaptive Regeneration}

In addition to the LAR, we introduce a modified LAR. In the modified LAR, the scaling coefficient $\kappa_{i}$ is defined as

$$
\kappa_{i}:=\frac{\min \left(d_{M_{0}}^{2} \gamma_{B S-R S_{i}}, d_{M_{i}}^{2} \bar{\gamma}_{R S_{i}-U T}\right)}{d_{M_{i}}^{2} \bar{\gamma}_{R S_{i}-U T}}=\left\{\begin{array}{cl}
\frac{d_{M_{0}}^{2} \gamma_{B S-R S_{i}}}{d_{M_{i}}^{2_{R}} \bar{\gamma}_{R S_{i}-U T}} & \text { if } \gamma_{B S-R S_{i}}<\frac{d_{M_{i}}^{2}}{d_{M_{0}}^{2}} \bar{\gamma}_{R S_{i}-U T} \\
1 & \text { if } \gamma_{B S-R S_{i}} \geq \frac{d_{M_{i}}^{2}}{d_{M_{0}}^{2}} \bar{\gamma}_{R S_{i}-U T}
\end{array}\right.
$$

where $d_{M_{i}}^{2}$ is given by (3.3). Note that the complexity of both the LAR and modified LAR is the same. The rational behind the modification is to account for the difference in reliability introduced by having the modulation level in RS different than the modulation level in BS. Note that the min operation used in (5.2) compares the SNRs only. We chose $d_{M_{i}}^{2}$ pragmatically to weight the SNRs before the comparison. We conjecture that $d_{M_{i}}^{2}$ is the weight to be used when comparing SNRs of different 
modulation levels. Our conjecture is based on the fact that the output SNR of the SBMRC is equal to the weighted sum of the individual SNRs, and these weights are equal to $d_{M_{i}}^{2}$, as given in (4.28). Although this modification is solely based on intuition, it is shown in Section 5.5 that it provides significant gain compared to LAR. Note that the modified LAR reduces to LAR if all the transmitting nodes use the same modulation level.

\subsection{Performance Analysis of LAR and modified LAR}

In $[19,37]$, it is only proven that LAR achieves full diversity. In this section, we find a closed-form approximation of the average BER of the LAR and modified LAR, for the general case when the BS and RS use different modulation schemes. For mathematical tractability, we consider the case of a single RS $(L=2)$. To generalize the derivations for both LAR and modified LAR, we write $\kappa$ as

$$
\begin{gathered}
\kappa=\left\{\begin{array}{cc}
\frac{\gamma_{B S-R S}}{\rho \bar{\gamma}_{R S-U T}} & \text { if } \gamma_{B S-R S}<\rho \bar{\gamma}_{R S-U T} \\
1 & \text { if } \gamma_{B S-R S} \geq \rho \bar{\gamma}_{R S-U T}
\end{array}\right. \\
\text { where } \rho=\left\{\begin{array}{cc}
1 & \text { LAR } \\
\frac{d_{M_{1}}^{2}}{d_{M_{0}}^{2}} \text { Modified LAR }
\end{array}\right.
\end{gathered}
$$

We start by evaluating the instantaneous BER for a given instantaneous SNR triplet $\left(\gamma_{B S-R S}, \gamma_{B S-U T}, \gamma_{R S-U T}\right)$ in Section 5.4.1. Then, in Section 5.4.2, we find the average BER by averaging $B E R_{\text {inst }}$ over the distribution of the SNR triplet and express the average BER as a function of the average SNR triplet $\left(\bar{\gamma}_{B S-R S}, \bar{\gamma}_{B S-U T}, \bar{\gamma}_{R S-U T}\right)$.

\subsubsection{Finding the instantaneous BER}

The instantaneous BER at the UT can be written as [24]

$$
B E R_{\text {inst }}=\left(1-B E R^{B S-R S}\right) B E R^{c o o p}+B E R^{B S-R S} B E R^{\text {prop }}
$$


where

$B E R^{B S-R S}$ : The instantaneous BER in the BS - RS link

$B E R^{c o o p}:$ The instantaneous BER at the UT after combining using SBMRC, given that no error happened at the RS

$B E R^{\text {prop }}$ : The instantaneous BER at the UT after combining using SBMRC, given that the RS has detection error.

The term $B E R^{B S-R S}$ is well approximated by [21]

$$
B E R_{B S-R S}=c_{M_{0}} Q\left(\sqrt{2 d_{M_{0}}^{2} \gamma_{B S-R S}}\right)
$$

where $c_{M_{0}}$ and $d_{M_{0}}$ are given by (3.3).

A tight lower bound of $B E R^{c o o p}$ is derived in Section 4.3.2 and given by (4.29). Thus, $B E R^{c o o p}$ can be written as

$$
B E R^{c o o p} \approx \tau Q\left(\sqrt{2 \gamma_{o u t}}\right), \gamma_{\text {out }}=d_{M_{0}}^{2} \gamma_{B S-U T}+\kappa d_{M_{1}}^{2} \gamma_{R S-U T}
$$

where $\tau=0.75$ for combining QPSK and 16-QAM or combing 16-QAM and 16-QAM, $\tau=0.5833$ for combining QPSK and 64-QAM or combining 64-QAM and 64-QAM and $\tau=0.5$ for combining 16-QAM and 64-QAM.

It is well known that finding $B E R^{\text {prop }}$ for multilevel modulations (such as $\mathrm{M}$ QAM) is quite difficult even if the signals belong to the same modulation level [24]. Therefore, we introduce the following lower bound. The RS is assumed not to transmit if an error occurs. Thus, errors will happen only in the BS-UT link. As a result, we can write the following

$$
B E R^{p r o p}>c_{M_{0}} Q\left(\sqrt{2 d_{M_{0}}^{2} \gamma_{B S-U T}}\right)
$$

The previous bound is very tight in the case where $\bar{\gamma}_{B S-R S} \ll \bar{\gamma}_{R S-U T}$, since $\kappa \approx 0$. The bound becomes looser when $\bar{\gamma}_{B S-R S} \gg \bar{\gamma}_{R S-U T}$; however, in this scenario, (1 - 
$\left.B E R^{B S-R S}\right) B E R^{c o o p} \gg B E R^{B S-R S} B E R^{\text {prop }}$ which makes the term $B E R^{B S-R S} B E R^{\text {prop }}$ negligible in the end-to-end BER performance. As it will be shown later, introducing such a bound gives sufficiently accurate results as long as $\bar{\gamma}_{B S-R S}>\bar{\gamma}_{R S-U T}$.

\subsubsection{Finding the average BER}

Using (5.5), (5.6), (5.7), and (5.8), the average BER can be written as

$$
\begin{gathered}
B E R>I_{0}+I_{1}, \text { where } \\
I_{0}=\int_{0}^{\infty} \int_{0}^{\infty}\left(1-c_{M_{0}} Q\left(\sqrt{2 d_{M_{0}}^{2} \gamma_{B S-R S}}\right)\right) \tau Q\left(\sqrt{2 \gamma_{\text {out }}}\right) \times \\
f_{\gamma_{\text {out }}}\left(\gamma_{\text {out }}\right) f_{\gamma_{B S-R S}}\left(\gamma_{B S-R S}\right) d \gamma_{\text {out }} \mathrm{d} \gamma_{B S-R S} \\
I_{1}=\int_{0}^{\infty} \int_{0}^{\infty} c_{M_{0}} Q\left(\sqrt{2 d_{M_{0}}^{2} \gamma_{B S-R S}}\right) c_{M_{0}} Q\left(\sqrt{2 d_{M_{0}}^{2} \gamma_{B S-U T}}\right) \times \\
f_{\gamma_{B S-R S}}\left(\gamma_{B S-R S}\right) f_{\gamma_{B S-R S}}\left(\gamma_{B S-U T}\right) d \gamma_{B S-R S} d \gamma_{B S-U T} .
\end{gathered}
$$

Since the channel coefficients are modeled as independent Rayleigh fading random variables, $\gamma_{B S-R S}, \gamma_{B S-U T}$, and $\gamma_{R S-U T}$ are modeled as independent exponential random variables $[22,23]$ and can be written as

$$
\begin{aligned}
& f_{\gamma_{B S-R S}}\left(\gamma_{B S-R S}\right)=\frac{1}{\bar{\gamma}_{B S-R S}} e^{-\frac{\gamma_{B S-R S}}{\bar{\gamma}_{B S-R S}} u\left(\gamma_{B S-R S}\right)} \\
& f_{\gamma_{B S-U T}}\left(\gamma_{B S-U T}\right)=\frac{1}{\bar{\gamma}_{B S-U T}} e^{-\frac{\gamma_{B S-U T}}{\bar{\gamma}_{B S-U T}} u\left(\gamma_{B S-U T}\right)} \\
& f_{\gamma_{R S-U T}}\left(\gamma_{R S-U T}\right)=\frac{1}{\bar{\gamma}_{R S-U T}} e^{-\frac{\gamma_{R S-U T}}{\bar{\gamma}_{R S-U T}} u\left(\gamma_{R S-U T}\right) .}
\end{aligned}
$$

For a given $\kappa, \gamma_{o u t}$ at the output of SBMRC is a sum of two independent exponential random variables; hence, its PDF is given by [23]

$$
f_{\gamma_{\text {out }}}\left(\gamma_{\text {out }}\right)=\frac{1}{d_{M_{0}}^{2} \bar{\gamma}_{B S-U T}-d_{M_{1}}^{2} \kappa \bar{\gamma}_{R S-U T}}\left(e^{-\frac{\gamma_{\text {out }}}{d_{M_{0}}^{2} \bar{\gamma}_{B S-U T}}}-e^{-\frac{\gamma_{M_{1}}^{2} \kappa \bar{\gamma}_{R S-U T}}{d^{2}}}\right) u\left(\gamma_{\text {out }}\right) .
$$


By substituting (5.12) and (5.13) in (5.10) and (5.11), and evaluating the integrals, $I_{0}$ and $I_{1}$ can be expressed as (the detailed derivations are given in Appendix C)

$$
\begin{aligned}
& I_{0}=\sum_{n=0}^{1} \sum_{m=0}^{1} P_{m, n}+ \\
& e^{-\frac{\rho \bar{\gamma}_{R S-U T}}{\bar{\gamma}_{B S-U T}}} Q\left(\sqrt{2 d_{M_{0}}^{2} \frac{\rho \bar{\gamma}_{R S-U T}}{\bar{\gamma}_{B S-R S}}}\right)-\sqrt{\frac{d_{M_{0}}^{2} \bar{\gamma}_{B S-R S}}{1+d_{M_{0}}^{2} \bar{\gamma}_{B S-R S}}} Q\left(\sqrt{\frac{\rho \bar{\gamma}_{R S-U T}\left(1+d_{M_{0}}^{2} \bar{\gamma}_{B S-R S}\right)}{\bar{\gamma}_{B S-R S}}}\right) \times \\
& \left(d_{M_{0}}^{2} \bar{\gamma}_{B S-U T}\left(1-\sqrt{\frac{d_{M_{0}}^{2} \bar{\gamma}_{B S-U T}}{1+d_{M_{0}}^{2} \bar{\gamma}_{B S-U T}}}\right)-d_{M_{1}}^{2} \bar{\gamma}_{R S-U T}\left(1-\sqrt{\frac{d_{M_{1}}^{2} \bar{\gamma}_{R S-U T}}{1+d_{M_{1}}^{2} \bar{\gamma}_{R S-U T}}}\right)\right) \times \\
& \frac{1}{d_{M_{0}}^{2} \bar{\gamma}_{B S-U T}-d_{M_{1}}^{2} \bar{\gamma}_{R S-U T}} \\
& P_{m, n}=\frac{\rho a_{m} \tau}{2 \bar{\Gamma}_{2}\left(\bar{\Gamma}_{0}+1\right)} e^{\frac{\rho}{\bar{\Gamma}_{2}}}\left(\begin{array}{l}
\operatorname{Ei}\left(\frac{\rho\left(\bar{\Gamma}_{1}+1\right)}{\bar{\Gamma}_{2}}\right)-\operatorname{Ei}\left(\frac{\rho}{\bar{\Gamma}_{2}}\right)+ \\
2 a_{n} c_{M_{0}} e^{\frac{\rho b_{n} d_{M_{0}}^{2} d_{M_{1}}^{2}}{b_{1}}}\left(\operatorname{Ei}\left(\frac{\rho\left(\bar{\Gamma}_{3}+1\right)}{\bar{\Gamma}_{2}}\right)-\operatorname{Ei}\left(\frac{\rho\left(\bar{\Gamma}_{3}+1\right)\left(\bar{\Gamma}_{1}+1\right)}{\bar{\Gamma}_{2}}\right)\right)
\end{array}\right), \\
& \bar{\Gamma}_{0}=b_{m} d_{M_{0}}^{2} \bar{\gamma}_{B S-U T}, \bar{\Gamma}_{1}=b_{m} d_{M_{1}}^{2} \bar{\gamma}_{R S-U T}, \bar{\Gamma}_{2}=b_{m} d_{M_{1}}^{2} \bar{\gamma}_{B S-R S}, \bar{\Gamma}_{3}=b_{n} d_{M_{0}}^{2} \bar{\gamma}_{B S-R S}, \\
& a_{0}=\frac{1}{12}, a_{1}=\frac{1}{4}, b_{0}=1 \text {, and } b_{1}=\frac{4}{3} \text {. } \\
& I_{1}=\frac{1}{4}\left(1-\sqrt{\frac{d_{M_{0}}^{2} \bar{\gamma}_{B S-R S}}{1+d_{M_{0}}^{2} \bar{\gamma}_{B S-R S}}}\right)\left(1-\sqrt{\frac{d_{M_{0}}^{2} \bar{\gamma}_{R S-U T}}{1+d_{M_{0}}^{2} \bar{\gamma}_{R S-U T}}}\right) .
\end{aligned}
$$

\subsection{Analytical and Simulation Results}

In this section, we present analytical and simulation results of the BER performance of simple DF, LAR and modified LAR ${ }^{1}$.

We first consider relay networks with $L=2$ (single relay), and for the sake of presentation, we assume the average channel conditions to be the same in all the links, i.e., $\bar{\gamma}_{B S-R S}=\bar{\gamma}_{B S-U T}=\bar{\gamma}_{R S-U T}=\bar{\gamma}$. Figure 5.1 shows the BER performance of SBMRC when $\left(M_{0}=4, M_{1}=16\right)$ and $\left(M_{0}=16, M_{1}=4\right)$ using simple DF, LAR and modified LAR. We also plot the BER of the fixed relay case. Note that

\footnotetext{
${ }^{1}$ See appendix $A$ for the detailed confidence interval calculation.
} 
in the fixed relay case both $\left(M_{0}=4, M_{1}=16\right)$ and $\left(M_{0}=16, M_{1}=4\right)$ have the same BER performance. For all error mitigation strategies, the BER performance is always better for the case when $\left(M_{0}=4, M_{1}=16\right)$. Note that the end-to-end spectral efficiency for both $\left(M_{0}=4, M_{1}=16\right)$ and $\left(M_{0}=16, M_{1}=4\right)$ is the same and equal to $\delta=1.33$. Consequently, it is always better to transmit using $\left(M_{0}=4\right.$, $\left.M_{1}=16\right)$ rather than $\left(M_{0}=16, M_{1}=4\right)$. This fact was verified through extensive simulations for different SNR triplet $\left(\bar{\gamma}_{B S-R S}, \bar{\gamma}_{B S-U T}, \bar{\gamma}_{R S-U T}\right)$. Moreover, significant performance loss is observed when the relay always retransmits in simple DF, and the diversity order is unity, as opposed to all other error mitigation strategies. This loss came as a result of the error propagation. Although both the LAR and modified LAR achieve full diversity, the modified LAR achieves a gain of $0.5 \mathrm{~dB}$ at BER of $10^{-3}$, compared to the LAR for the case $\left(M_{0}=4, M_{1}=16\right)$. This is a significant gain that comes as a result of modifying the power scaling coefficient. Note that the gap between the fixed RS and the modified LAR is $2 \mathrm{~dB}$, which suggests that there is still space for improvement and designing a better error mitigation strategy. In Figs. 5.2 and 5.3, we show the BER performance for combining QPSK and 64QAM, and combining 16-QAM and 64-QAM, respectively. The same conclusions drawn from Fig. 5.1 are observed. We observe that in general, it is always better to set $M_{0} \leq M_{1}$, if the modulation assignments are done at the BS based on the average channel conditions. In other words, it is crucial to assign the most reliable modulation in the link from BS to RS, to reduce the effect of error propagation as the performance of digital relaying is limited by the errors made at the relay. We give an explicit analytical proof for this in Appendix D. The gain at BER of $10^{-3}$, achieved by using the modified LAR over the LAR for the cases $\left(M_{0}=4, M_{1}=64\right)$ and $\left(M_{0}=16, M_{1}=64\right)$, are $0.8 \mathrm{~dB}$ and $0.9 \mathrm{~dB}$, respectively. 
In Fig. 5.4, we consider relay networks with $L=3$ (two relays). For the sake of presentation, we assume the average channel conditions to be the same in all the links, i.e., $\bar{\gamma}_{B S-R S_{1}}=\bar{\gamma}_{B S-R S_{2}}=\bar{\gamma}_{B S-U T}=\bar{\gamma}_{R S_{1}-U T}=\bar{\gamma}_{R S_{2}-U T}=\bar{\gamma}$. Again, the diversity order of the simple DF is 1 while the LAR and modified LAR achieve diversity order of 3. The modified LAR in this scenario achieves a modest gain of $0.2 \mathrm{~dB}$ at BER of $10^{-3}$. However, the gain increases to $0.8 \mathrm{~dB}$ at BER of $10^{-5}$.

In Figs. 5.5 and 5.6, we plot the analytical lower bound of the BER as well as the simulation results for different scenarios, for both LAR and modified LAR, respectively. It is clear from the figure that the derived lower bound given by (5.9) is very tight. In Fig. 5.7, we plot the BER of both LAR and modified LAR as a function of $\bar{\gamma}_{B S-R S}$ for the case when $\left(M_{0}=4, M_{1}=16\right)$. From the figure, it is clear that the modified LAR has better BER performance. Moreover, the developed lower bound is tighter for LAR. In addition, the lower bound is tight for both LAR and modified LAR as long as $\bar{\gamma}_{B S-R S}>\bar{\gamma}_{R S-U T}$. 


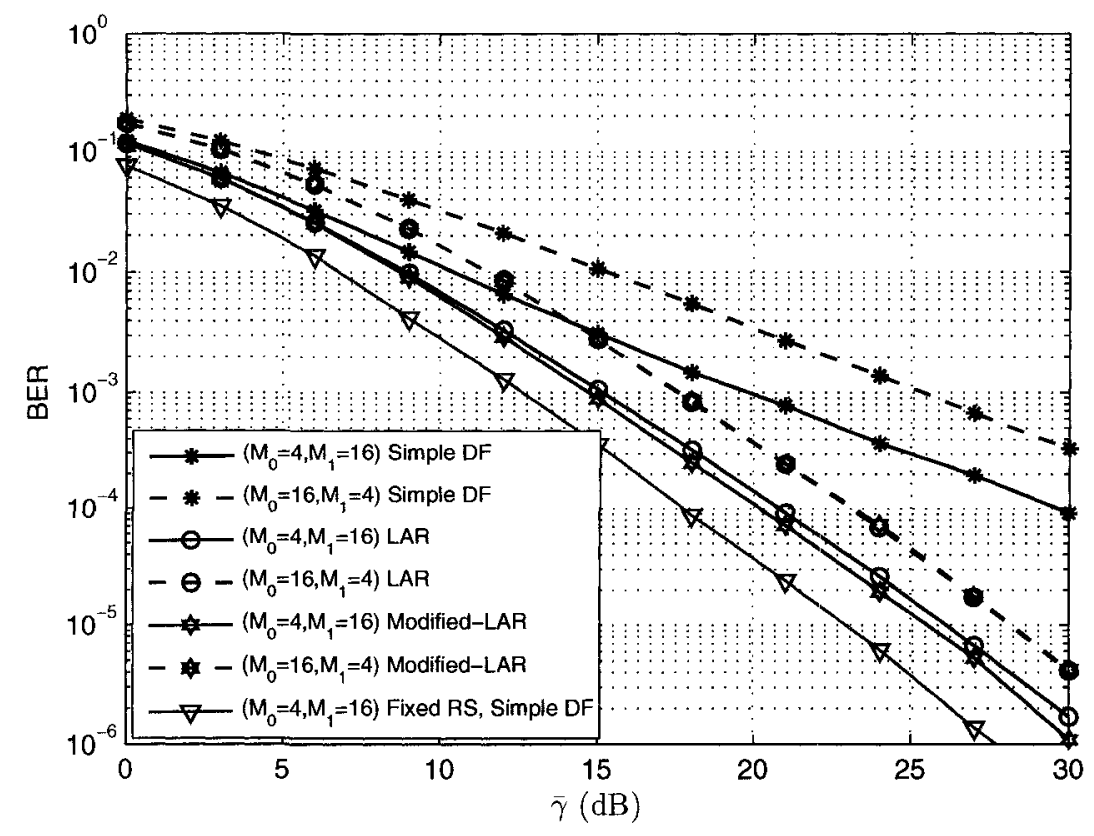

Figure 5.1: BER performance of combining QPSK and 16-QAM using SBMRC for different strategies.

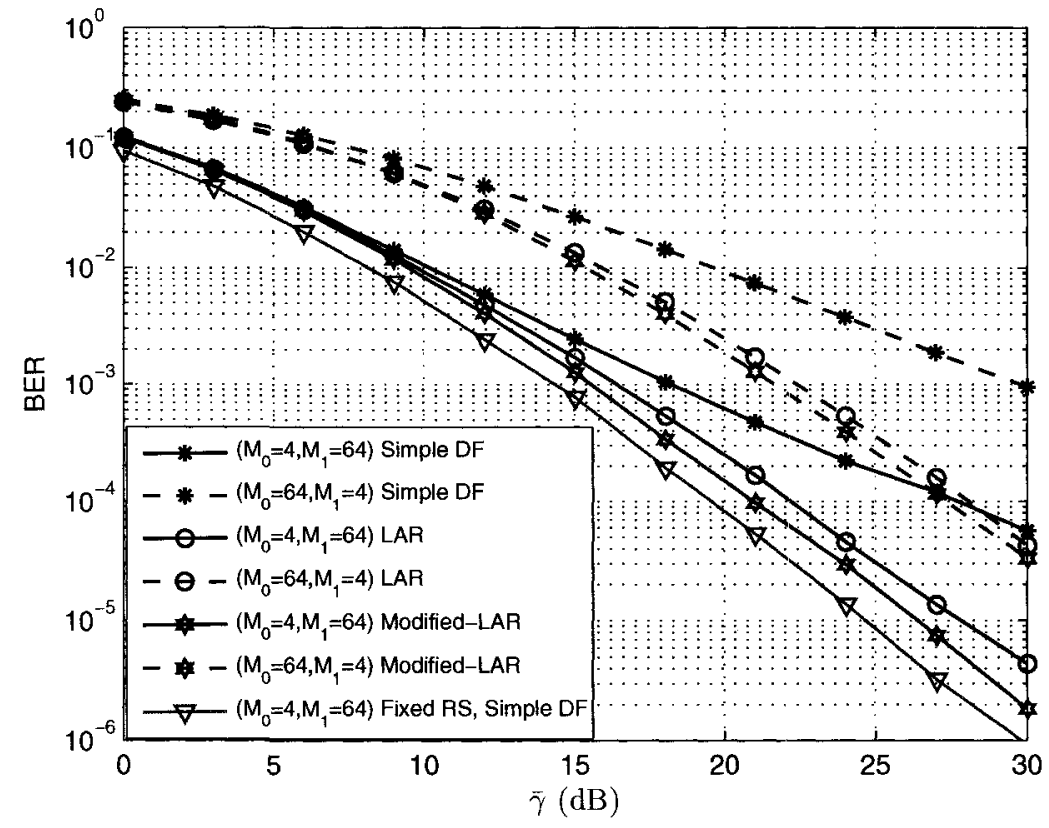

Figure 5.2: BER performance of combining QPSK and 64-QAM using SBMRC for different strategies. 


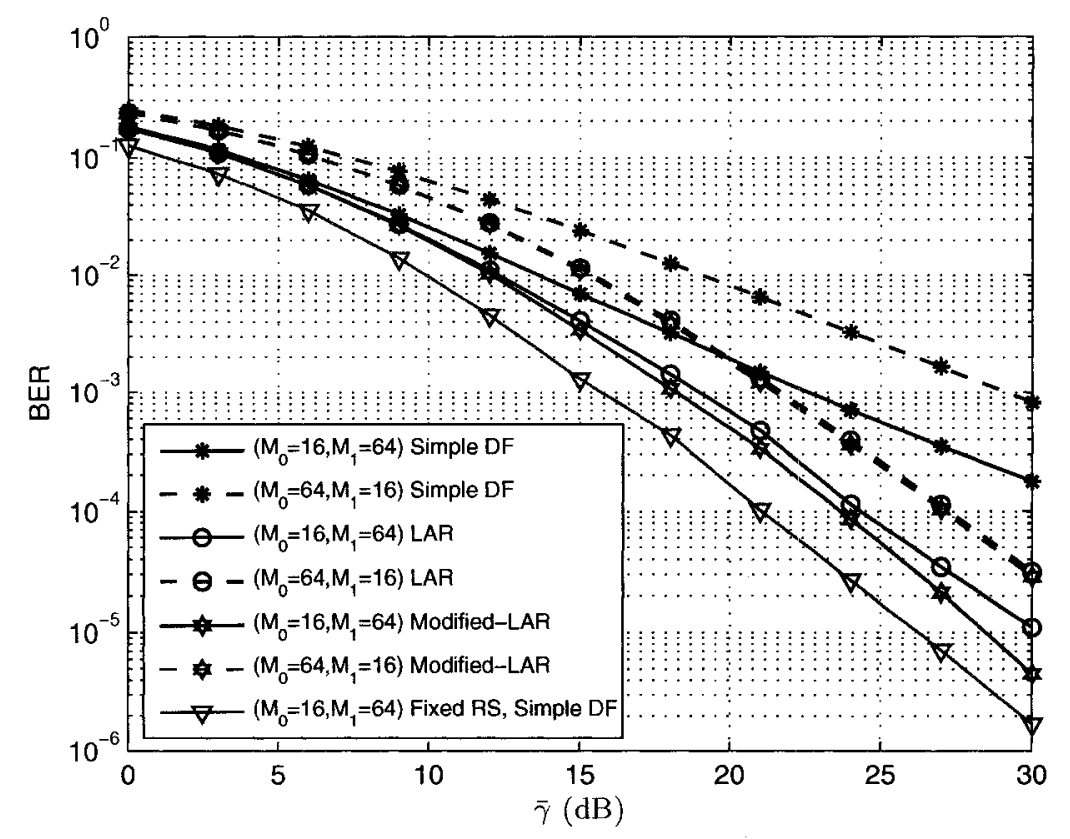

Figure 5.3: BER performance of combining 16QAM and 64-QAM using SBMRC for different strategies.

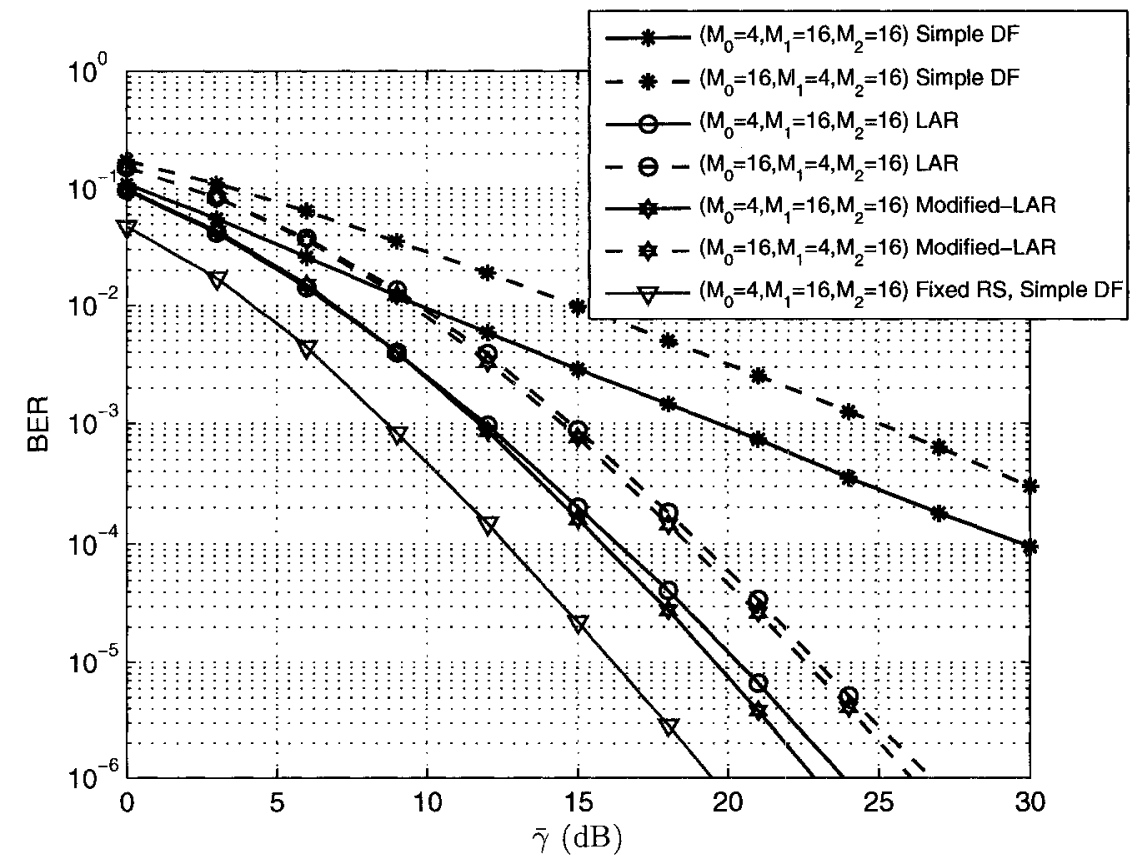

Figure 5.4: BER performance of combining QPSK, 16-QAM and 16-QAM using SBMRC for different strategies. 


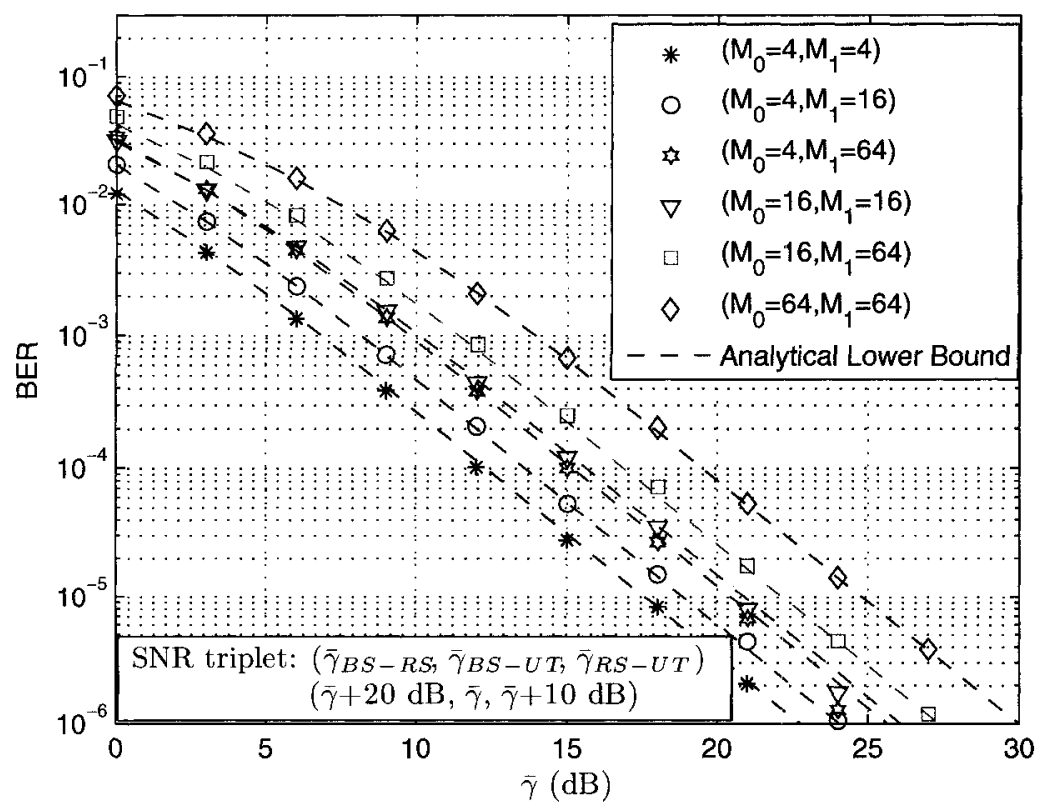

Figure 5.5: BER performance of LAR using both simulation and analytical lower bound.

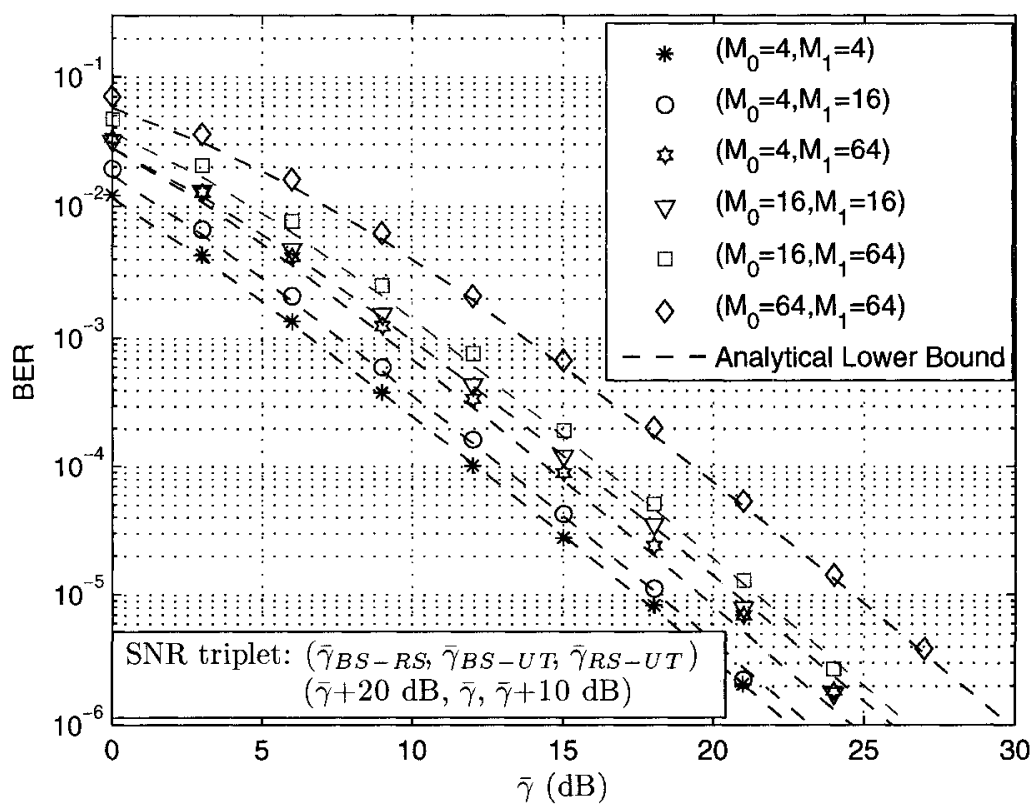

Figure 5.6: BER performance of modified LAR using both simulation and analytical lower bound. 


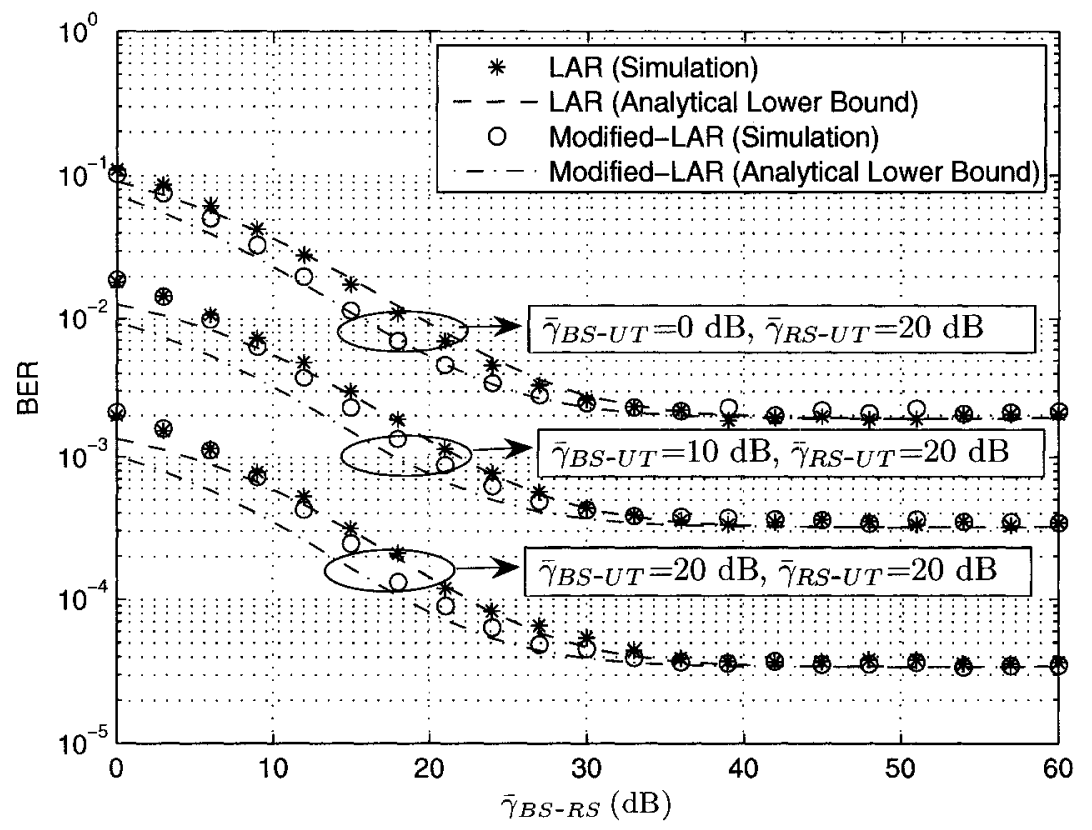

Figure 5.7: BER performance of both LAR and modified LAR as a function of $\bar{\gamma}_{B S-R S}$. 


\section{Chapter 6}

\section{Constellation Rearrangement (CoRe) in Cooperative Relay Networks}

In Chapters 3, 4, and 5, we studied the case when the BS and the RS use different modulation levels. In this chapter, the same modulation level is used by both the BS and the RS but with different bit labeling in the constellation. Varying the bit labeling for retransmission is called CoRe and it is originally introduced in the context of HARQ in [14]. Thus, we will give a brief overview of HARQ and the existing literature in CoRe in Section 6.1.

\subsection{Review of Hybrid Automatic Repeat reQuest and Constellation Re- arrangement}

$A R Q$, an error control mechanism, constitutes an essential part in data communications that enables error free transmission of data packets through multiple transmissions. If the receiver detects an error in the packet, it asks the transmitter to retransmit the erroneously decoded packet. This process is repeated until the packet is decoded correctly, or the maximum allowed number of retransmissions occurs. HARQ improves the performance of ARQ by employing Forward Error Correction (FEC). HARQ is classified into Type I and Type II. In Type I, the erroneously decoded packets are discarded, while in Type II, these packets are stored and used for further decoding. One strategy in Type II is to retransmit the same identical bits 
of the original transmission and the receiver combines the original and the retransmitted packets using Chase combining. Other strategies include Full Incremental Redundancy and Partial Incremental Redundancy. [14]

In $[14,15]$, a scheme is proposed to average the variation in bit reliabilities inherited in the signal constellation of multilevel modulation such as PAM or QAM. In each retransmission, the transmitter use different Gray-coded constellations to transmit the same information bits. At the receiver, combining is done by adding the soft-bits since MRC does not work because the labeling is altered from one transmission to the next, despite the fact that all the retransmissions use the same modulation level. The benefit of this CoRe scheme is demonstrated for the modulations 16-QAM and 64-QAM. The mapping for the in-phase and quadrature competent of QAM constellation is orthogonal; consequently, it suffices to concentrate on the in-phase component. The labeling for the quadrature component is the same as that of the in-phase component. We call this scheme CoRe 1. In [18], it is realized that the performance of HARQ is similar to cooperative relaying in fixed relay environment, although in the latter the retransmission happens at the relay. Therefore, the CoRe concept can be applied to cooperative relaying as well. In this work, the authors applied CoRe 1 in cooperative relaying. Figures 6.1 and 6.2 show the in-phase labeling of CoRe 1 for two transmissions. Note that the constellations used for both transmissions are Gray-coded.

In [16], it is alluded that CoRe 1 is not optimized and indeed obtained in an "ad hoc" way. In this work, the authors propose an optimum bit to symbol mapping for an arbitrarily number of retransmissions. They fix the first transmission to a particular Gray-coded constellation, and through set partitioning and permutations, they find the bit to symbol mapping for next transmissions that maximizes the minimum 
squared Euclidean distance (SED). Aside from the first transmission, the next transmissions are not necessarily Gray-Coded. The gain of this scheme was illustrated using the concept of augmented signal constellation, which will be explained in detail in Sections 6.3 and 6.4. The labeling for the quadrature component is the same as that of the in-phase component. We call this scheme CoRe 2. In [17], CoRe 2 is used for multiple retransmissions combined with space-time block coding. Figs. 6.3 and 6.4 show the in-phase labeling of CoRe 2 for two transmissions. Note that while the constellation of the first transmission is Gray-coded, the constellation of the second transmission is not Gray-coded.

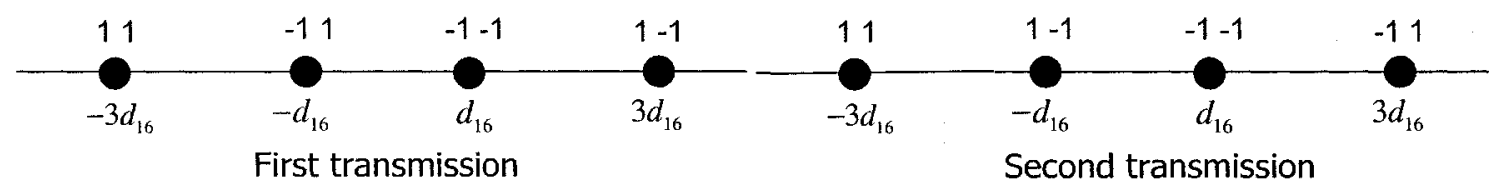

Figure 6.1: Bit labeling for the in-phase component of 16-QAM constellation for CoRe 1.

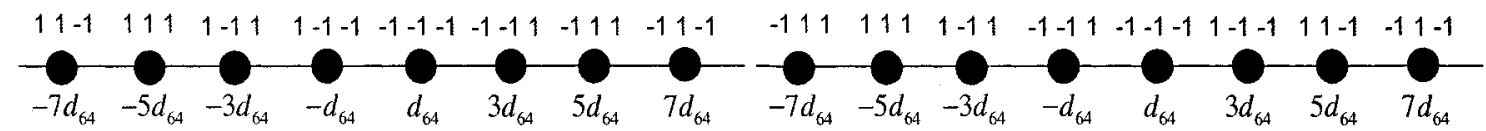

First transmission

Second transmission

Figure 6.2: Bit labeling for the in-phase component of 64-QAM constellation for CoRe 1. 


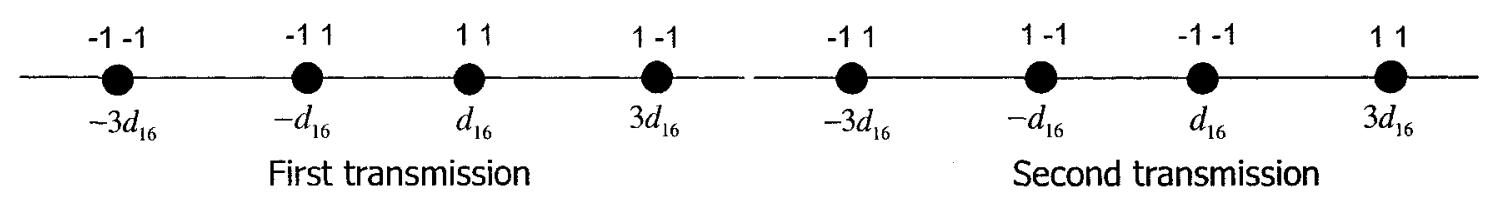

Figure 6.3: Bit labeling for the in-phase component of 16-QAM constellation for CoRe 2.

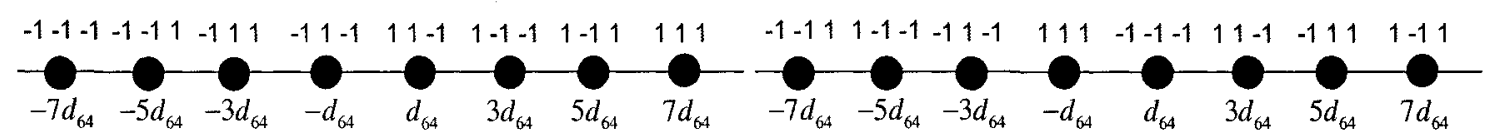

First transmission

Second transmission

Figure 6.4: Bit labeling for the in-phase component of 64-QAM constellation for CoRe 2 .

\subsection{System Model for CoRe in Cooperative Relaying}

In CoRe for cooperative relaying, we use the same system model presented in Chapter 2 with the following modifications. Since the modulation levels for all transmitting nodes are the same, then $M_{i}=M, K_{i}=K, C=L C M\left\{K_{0}, \ldots, K_{L-1}\right\}=K$, and $T_{i}=C / K_{i}=1$, for $i \in\{0, \ldots, L-1\}$. To simplify the notations, we drop $M_{i}$ and the index $j$ since $j \in\left\{0, \ldots, T_{i}-1\right\}$ which means that $j$ always equal to zero. The transmitting node $i$ (a BS or RS), transmits the M-QAM modulated symbol $S_{i}$, that use the bit-to-symbol mapping $i$. The symbols $S_{i}$, for $i \in\{0, \ldots, L-1\}$, contain the same information bits $\left\{s_{0}, s_{1}, \ldots, s_{K-1}\right\}$ and each is defined as

$$
S_{i}=f_{i}^{R}\left(s_{0}, \ldots, s_{K / 2-1}\right)+j f_{i}^{I}\left(s_{K / 2}, \ldots, s_{K-1}\right)
$$

where $f_{i}^{R}$ and $f_{i}^{I}$ define the mapping for the in-phase (real) and quadrature (imaginary) components of $S_{i}$, respectively. Similar to CoRe 1 and CoRe 2, we the assume that $f_{i}^{R}=f_{i}^{I}=f_{i}$. The design of $f_{i}$ is explained in the subsequent section.

The receiver is assumed as MLD, as in [16,17], and the decoding rule is defined 


$$
\begin{aligned}
& {\left[\hat{s}_{0}, \ldots, \hat{s}_{K-1}\right]=\arg \min _{s_{0}, \ldots, s_{K-1}} \sum_{i=0}^{L-1}\left|r_{i}-\alpha_{i} S_{i}\right|^{2} \Rightarrow} \\
& \left\{\begin{array}{l}
{\left[\hat{s}_{0}, \ldots, \hat{s}_{K / 2-1}\right]=\arg \min _{s_{0}, \ldots, s_{K / 2-1}} \sum_{i=0}^{L-1}\left|\mathcal{R}\left\{e^{-j \angle \alpha_{i}} r_{i}\right\}-\right| \alpha_{i}\left|f_{i}\left(s_{0}, \ldots, s_{K / 2-1}\right)\right|^{2}} \\
{\left[\hat{s}_{K / 2}, \ldots, \hat{s}_{K-1}\right]=\arg \min _{s_{K / 2}, \ldots, s_{K-1}} \sum_{i=0}^{L-1}\left|\mathcal{I}\left\{e^{-j \angle \alpha_{i}} r_{i}\right\}-\right| \alpha_{i}\left|f_{i}\left(s_{K / 2}, \ldots, s_{K-1}\right)\right|^{2}}
\end{array}\right.
\end{aligned}
$$

We remark that the MLD receiver is not as complex as in the case of combining different modulation levels discussed in Section 4.1. Indeed, in this case the MLD's complexity is in the order of $2 \times 2^{K / 2} / K=2^{K / 2+1} / K$ computations per bit,

as opposed to the case in Section 4.1, where the complexity is in the order of $2^{C} / C$ computations per bit. For example, for the case of 16-QAM and 64-QAM, the computation complexity is in the order of 2 and 2.67 computations per bit, respectively. This is certainly affordable with the modern technology. Note that in $[14,15,18]$, a suboptimum receiver was used for CoRe 1. For fair comparison, we will use the MLD for all different CoRe schemes, which makes the receiver a neutral factor in the comparison between the different CoRe schemes.

\subsection{Proposed CoRe for Cooperative Relaying}

Before explaining the proposed CoRe for cooperative relaying, we highlight the similarities and differences between HARQ and cooperative relaying. In both, retransmission of the same information occurs and diversity combining is performed at the receiver side [18]. However, cooperative relaying differ in the following points that were not mentioned in [18]:

1. In HARQ, the source does not know the number of retransmissions needed to deliver a packet a priori, and in some cases where the packet is decoded correctly from the first transmission, there is no need for any retransmission. Therefore, 
it makes sense to fix the first transmission to be Gray-coded, to minimize the possibility of retransmissions, as it is well known that Gray-coding is the optimum labeling that minimize BER for single transmission [23]. However, in cooperative relaying, the source knows a priori the number of retransmissions as it knows the number of relays involved in cooperation. For example, if cooperation happens through a single relay, the source knows that two transmissions are needed. Therefore, the condition of having the first transmission Gray-coded can be relaxed.

2. In cooperative relaying in the nomadic relays scenario, the information bits retransmitted from the relays may not necessarily be the same as those transmitted by the source because of the possibility of making errors in decoding the signal from the source. This is not the case in HARQ, where all retransmissions represent the same information bit. The presence of error propagation in cooperative relaying raises the question whether the concept of CoRe benefits or harms the performance of cooperative relaying through nomadic relays. We answer this question in detail in Section 6.5.

The problem at hand is to find good mapping function $f_{i}$ for $i \in\{0, \ldots, L-1\}$. Due to the orthogonality of the labeling of the in-phase and quadrature components, we will concentrate on designing $f_{i}$ for the in-phase component that represents $\left.\sqrt{(} M\right)$ PAM, and the same design will be used for the quadrature component, to construct $M$-QAM labeling. Similar to [16], we define the augmented signal constellation or augmented signal space, $\Omega$, which is defined as the set of $M$ distinct $L$ dimensional vectors. The $\Omega$ modulated symbol is denoted by the $L$ dimensional vector $\mathbf{S}$. The 
one-to-one mapping between the bits $\left\{s_{0}, s_{1}, \ldots, s_{K-1}\right\}$ and $\mathbf{S}$ is implicitly defined as

$$
\mathrm{S}=\left(S_{0}, S_{1}, \ldots, S_{L-1}\right)
$$

where the mapping between $S_{i}$ and $\left\{s_{0}, s_{1}, \ldots, s_{K-1}\right\}$ is defined in (6.1). The reason of defining the augmented signal constellation in such a manner comes as result of the orthogonality of the different transmissions, which in turn make the constellation $L$ dimensional. We emphasize that this is a virtual constellation and it is not used by the transmitting nodes. It is used as a design and illustrating tool.

Let us label all possible signal points as $\mathbf{S}^{0}, \mathbf{S}^{1}, \ldots, \mathbf{S}^{M-1}$. The design criterion in designing the constellation is to maximize the minimum SED. Mathematically speaking, this can be written as

$$
\max _{j, k} \min \left\{\left\|\mathbf{S}^{j}-\mathbf{S}^{k}\right\|^{2}, \forall \mathbf{S}^{j}, \mathbf{S}^{k} \in \Omega, j \neq k\right\}
$$

We deal with the previous optimization problem using numerical exhaustive search by examining all possible combinations of $\mathbf{S}^{0}, \mathbf{S}^{1}, \ldots, \mathbf{S}^{M-1}$. The combinations are generated by finding all possible permutations of the signal points of $S_{i}$. Since $S_{i}$ is drawn from $\sqrt{M}$-PAM, the signal points are $\left\{(-\sqrt{M}+1) d_{M},(-\sqrt{M}+3) d_{M},(-\sqrt{M}+\right.$ 5) $\left.d_{M}, \ldots,(-\sqrt{M}+2 \sqrt{M}-1) d_{M}\right\}$. For example, for $\sqrt{16}$-PAM, the signal points are

$$
\left\{-3 d_{16},-d_{16}, d_{16}, 3 d_{16}\right\}
$$

and for $\sqrt{64}-\mathrm{PAM}$, the signal points are

$$
\left\{-7 d_{64},-5 d_{64},-3 d_{64},-d_{64}, d_{64}, 3 d_{64}, 5 d_{64}, 7 d_{64}\right\}
$$

Thus, we search over $(\sqrt{M} !)^{L}$ different combinations. For each combination, the SED is calculated and we pick that constellation that satisfies (6.4). We remark that the numerical search process is done only once while off-line and the best constellation 
is stored and used by the transmitting nodes. Thus, the computation complexity is less of an issue.

Up to this point, the procedure of finding the best augmented signal constellation is the same as that in [16]. The difference between the proposed CoRe and CoRe 2, is that in CoRe 2 (which was proposed for HARQ), the labeling is done using Graycoding for the first transmission. By doing so, the signal points in the augmented signal constellation are not Gray-coded, which means that the adjacent signal points in the augmented space differ by more than one bit. In the proposed CoRe, we do the labeling in the augmented space such as the adjacent points are Gray-coded. However, this results in having the first transmission not Gray-coded. As stated earlier, this is not a concern for fixed cooperative relay networks.

By performing the exhaustive search, the proposed CoRe labelings for the inphase component of 16-QAM and 64-QAM are shown in Figs. 6.5 and 6.6, respectively.

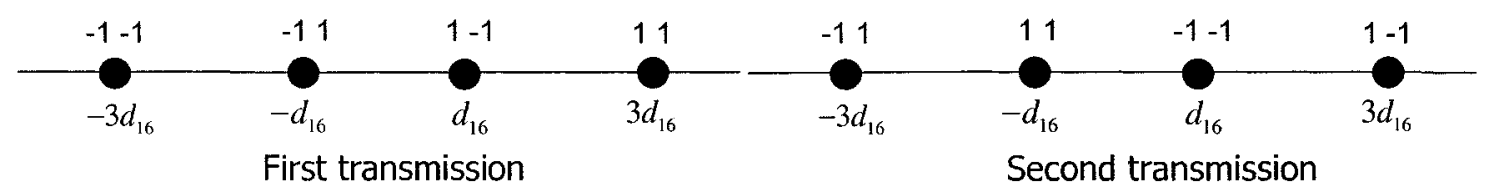

Figure 6.5: Bit labeling for the in-phase component of 16-QAM constellation for the proposed CoRe.

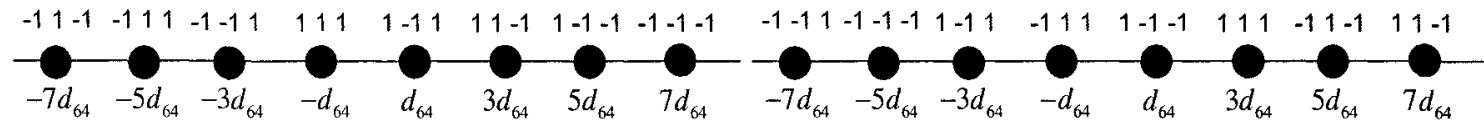

First transmission

Second transmission

Figure 6.6: Bit labeling for the in-phase component of 64-QAM constellation for the proposed CoRe. 


\subsection{Augmented Signal Constellation}

In this section, we give insightful comparison between conventional, CoRe 1 , CoRe 2, and the proposed CoRe using the concept of the augmented signal constellation for the case of two transmissions.

Figure 6.7 shows the augmented signal constellation for conventional scheme, where the same labeling is used for 4-PAM (the in-phase component for 16-QAM constellation). For example, if $(1,1)$ is to be transmitted, then in both the first and second transmission, $\left(-3 d_{16}\right)$ will be transmitted. Even though the available signal space is two dimensional, the points lie in a line, which means the points lie in a one-dimensional space. This suggests that the conventional scheme does not exploit the extra dimension achieved by the second transmission. The minimum Euclidian distance is $2 \sqrt{2} d_{16}$. The same conclusion is observed for the case of 8-PAM shown in Fig. 6.8 and the minimum Euclidian distance is $2 \sqrt{2} d_{64}$.

In Fig. 6.9, we plot the augmented signal constellation for CoRe 1 using 4PAM. For example, if $(1,-1)$ is to be transmitted, then $\left(-d_{16}\right)$ and $\left(3 d_{16}\right)$ will be transmitted in the first and second transmissions, respectively. This scheme has the same minimum Euclidian distance as the conventional scheme except that the symbol $(1,1)$ has much less probability of error than the other symbols as its adjacent symbols are much further away than the minimum distance. This hints that CoRe 1 will have slight gain compared to the conventional scheme, as the performance is dominated by the minimum distance. Moreover, it shows that CoRe 1 does not exploit the extra dimension fully in spreading the signal points, which supports the sub-optimality observation made in [16]. Similar conclusions can be made for CoRe 1 using 8-PAM shown in Fig. 6.10. 
In Fig. 6.11, we plot the augmented signal constellation for CoRe 2 using 4PAM. For example, if $(1,-1)$ is to be transmitted, then $\left(3 d_{16}\right)$ and $\left(-d_{16}\right)$ will be transmitted in the first and second transmission, respectively. It is clear that this scheme exploits the extra dimension very well, as the signal points are spread apart to achieve a minimum Euclidian distance of $2 \sqrt{5} d_{16}$, which is 1.6 larger than both the conventional and CoRe 1. Similar conclusions can be made for CoRe 2 using 8-PAM shown in Fig. 6.12.

In Fig. 6.13, we plot the augmented signal constellation for the proposed CoRe using 4-PAM. For example, if $(1,-1)$ is to be transmitted, then $\left(d_{16}\right)$ and $\left(3 d_{16}\right)$ will be transmitted in the first and second transmissions, respectively. Similar to CoRe 2 , this scheme exploits the extra dimension very well, as the signal points are spread apart to achieve a minimum Euclidian distance of $2 \sqrt{5} d_{16}$. However, it is better than CoRe 2, since the adjacent symbols differ only by single bit while in CoRe 2 some adjacent symbols differ by two bits. In other words, the proposed CoRe is Graycoded in the augmented signal constellation while CoRe 2 is only Gray-coded in the first tranmission. For the case of 8-PAM, the proposed CoRe achieves a minimum Euclidian distance of $2 \sqrt{8} d_{64}$ which is 1.3 times larger than CoRe 2 and 2 times larger than both CoRe 1 and the conventional schemes. Moreover, the proposed CoRe is Gray-coded in the augmented signal constellation, unlike CoRe 2. 


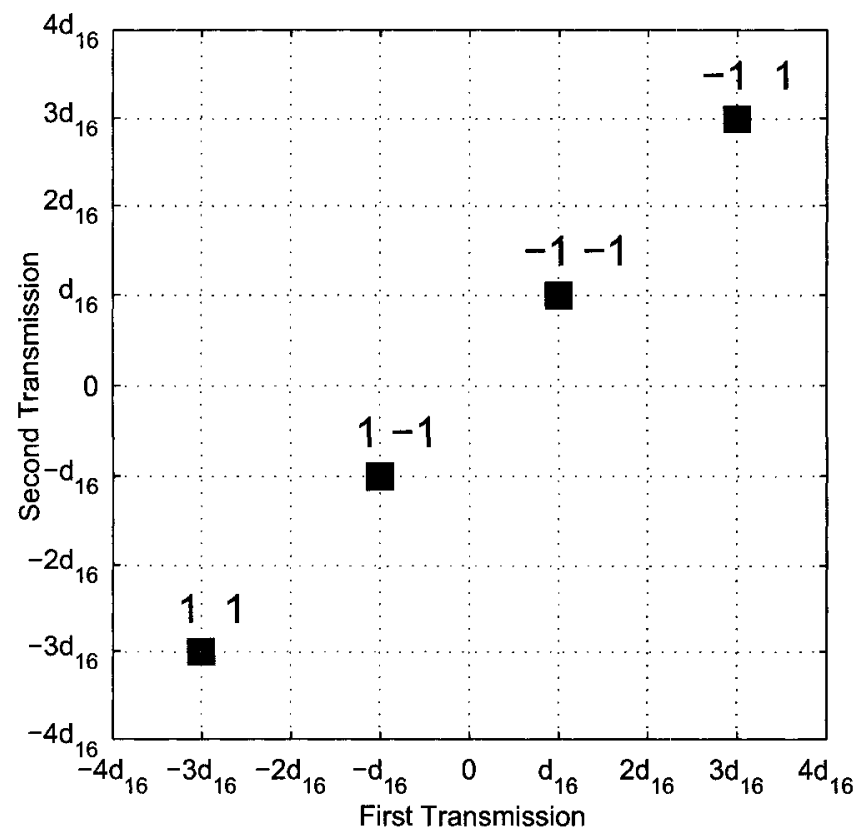

Figure 6.7: The augmented signal constellation of 4-PAM for conventional scheme.

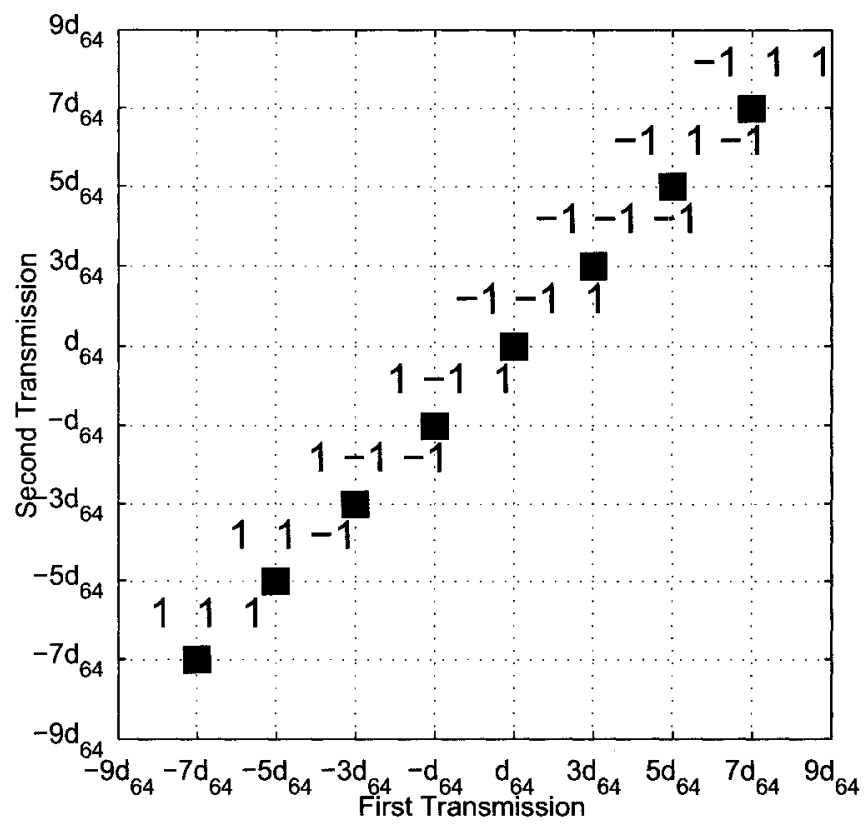

Figure 6.8: The augmented signal constellation of 8-PAM for conventional scheme. 


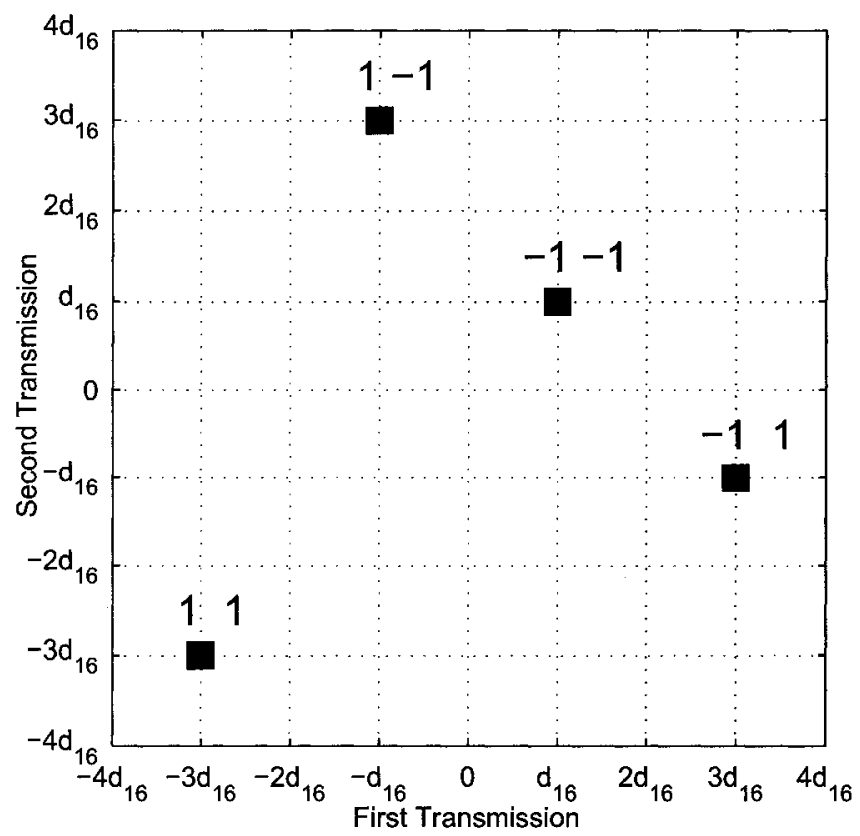

Figure 6.9: The augmented signal constellation of 4-PAM for CoRe 1.

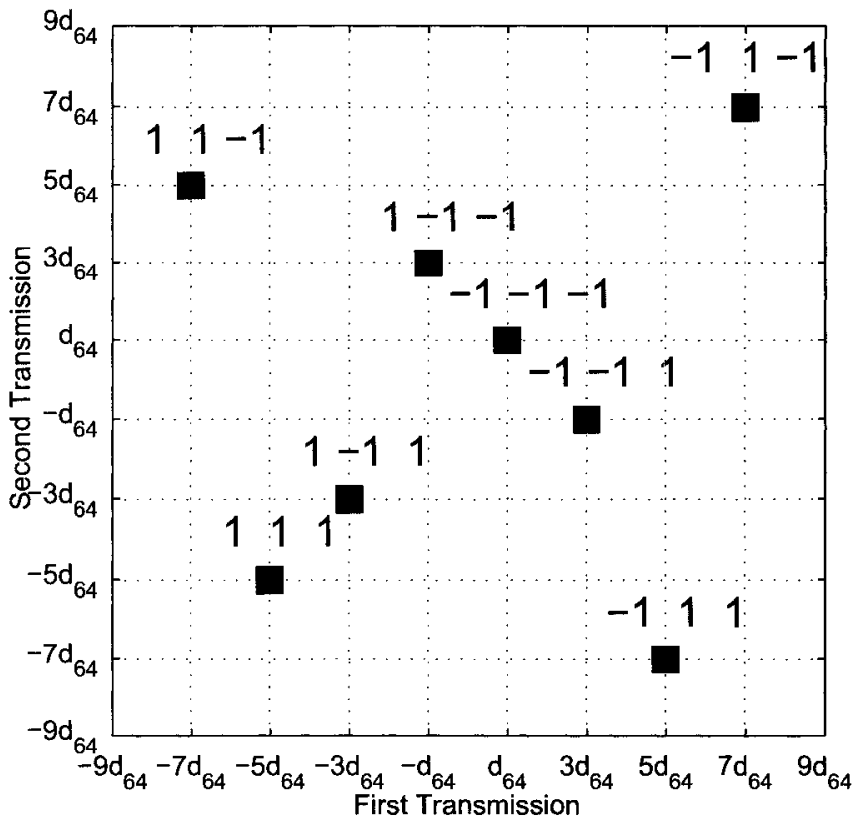

Figure 6.10: The augmented signal constellation of 8-PAM for CoRe 1. 


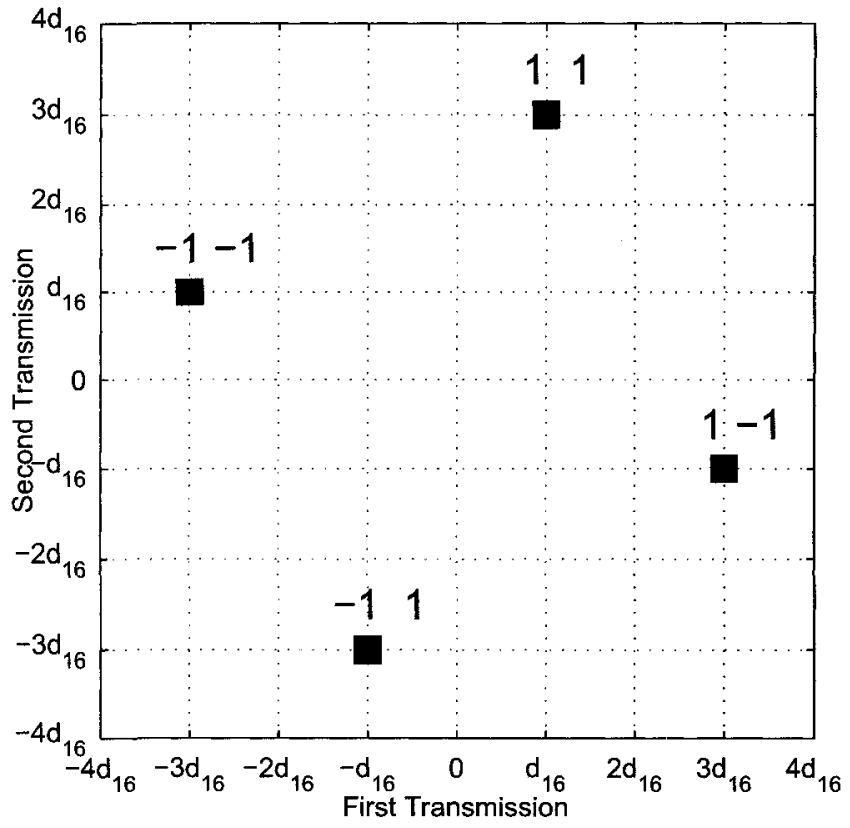

Figure 6.11: The augmented signal constellation of 4-PAM for CoRe 2.

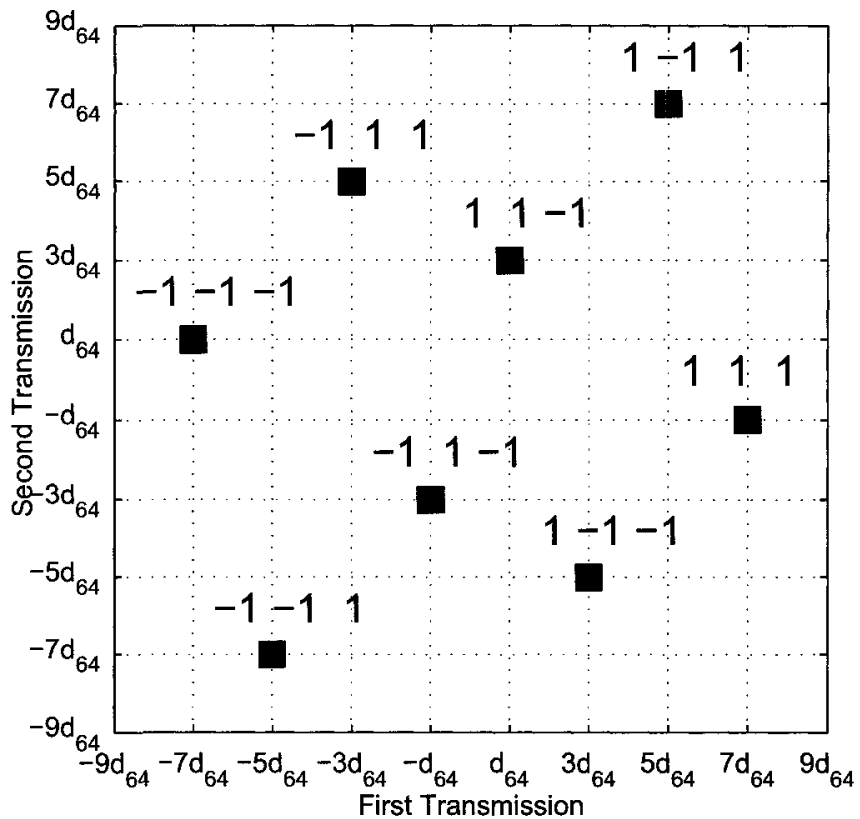

Figure 6.12: The augmented signal constellation of 8-PAM for CoRe 2. 


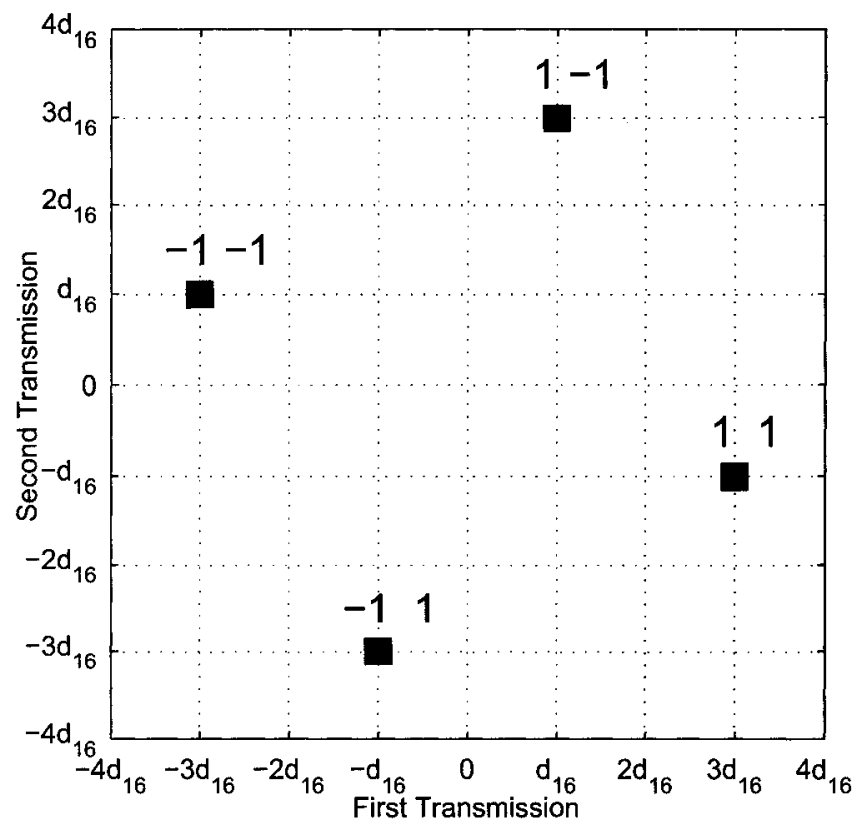

Figure 6.13: The augmented signal constellation of 4-PAM for the proposed CoRe.

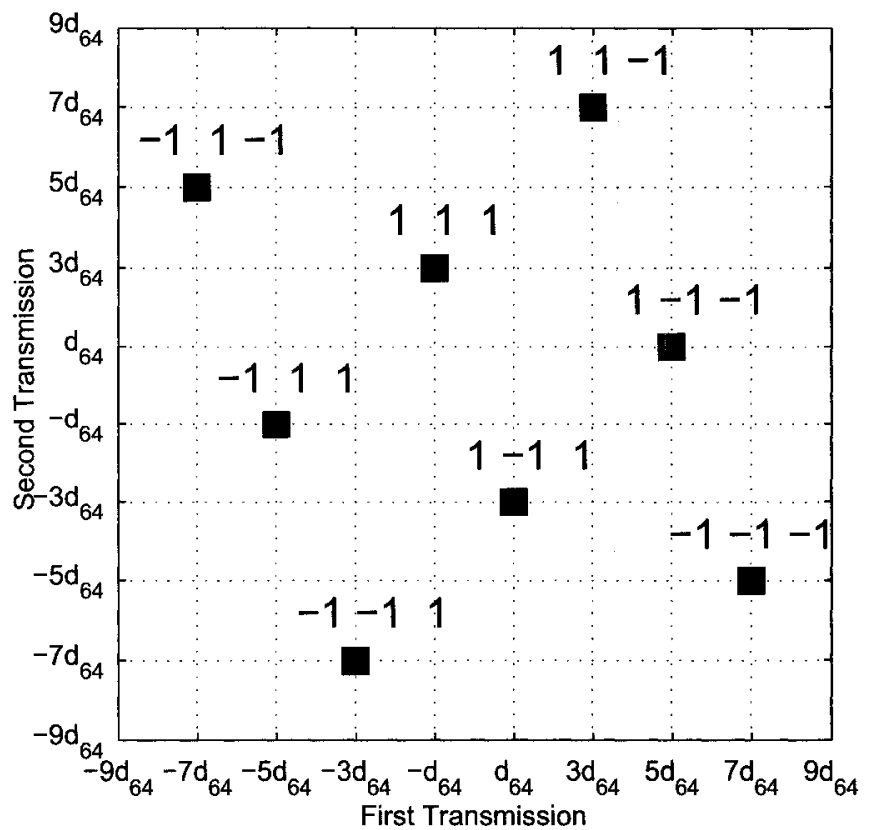

Figure 6.14: The augmented signal constellation of 8-PAM for the proposed CoRe. 


\subsection{Simulation Results}

To verify the conclusions made by observing the augmented signal constellation, we examine the BER results for the different schemes ${ }^{1}$.

In Fig. 6.15 and 6.16, we plot the BER curve for a single fixed relay network with $\bar{\gamma}_{B S-U T}=\bar{\gamma}_{R S-U T}=\bar{\gamma}$ for different CoRe schemes using 16-QAM and 64-QAM, respectively. In both figures, although all schemes achieve the same diversity order of 2, the proposed CoRe achieves the highest SNR gain. For the 16-QAM case, at a BER of $10^{-3}$, the proposed CoRe achieves gains of $2,1.25$, and 0.5 over the conventional, CoRe 1 and CoRe2 schemes, respectively. For the 64-QAM case, at a BER of $10^{-3}$, the proposed CoRe achieves gains of $3,1.5$ and 0.5 over the conventional, CoRe 1 and CoRe 2 schemes, respectively. These are significant gains, considering the simple processing involved to achieve them.

\footnotetext{
${ }^{1}$ See appendix $A$ for the detailed confidence interval calculation.
} 


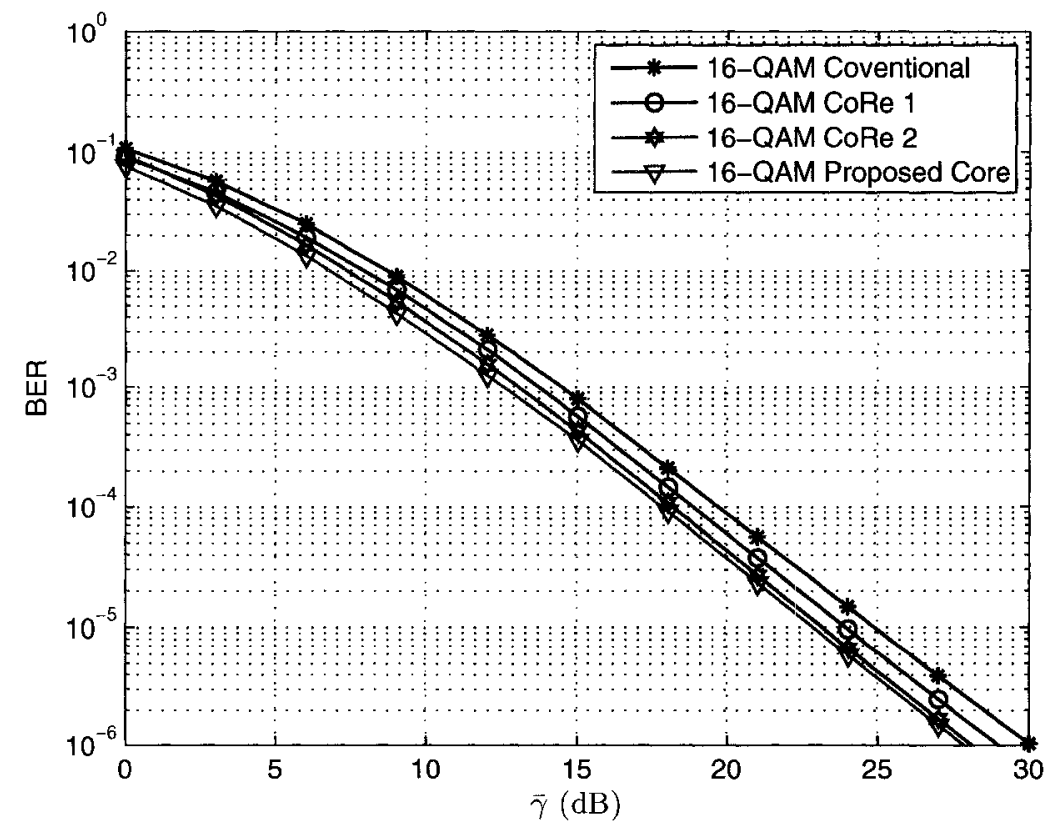

Figure 6.15: BER performance of different CoRe schemes in a fixed relay network using 16-QAM.

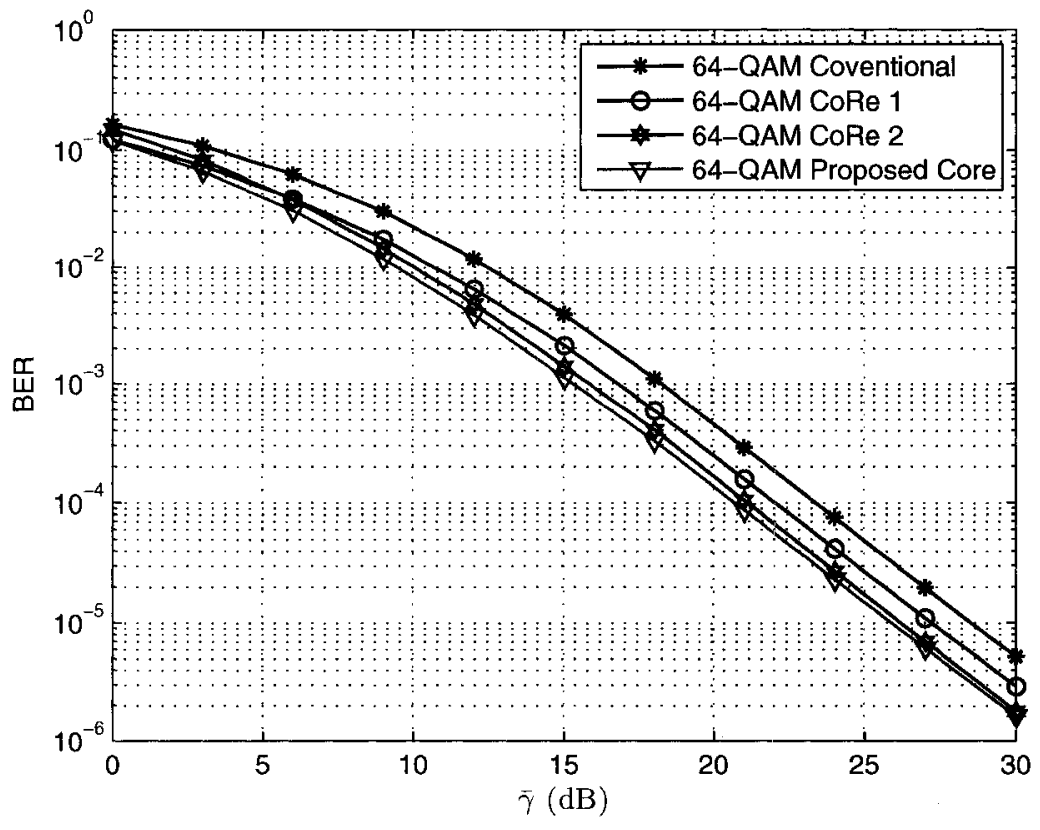

Figure 6.16: BER performance of different CoRe schemes in a fixed relay network using 64-QAM. 
Then we consider the case of a network with a single nomadic relay. We use the LAR strategy, discussed in Chapter 5, at the relay for all schemes. In Figs. 6.17 and 6.18, we plot the BER curve for different CoRe schemes, using 16-QAM and 64-QAM, respectively. In both figures, we consider the cases when the average SNRs $\left(\bar{\gamma}_{B S-R S}\right.$, $\left.\bar{\gamma}_{B S-U T}, \bar{\gamma}_{R S-U T}\right)$ are equal to $(\bar{\gamma}, \bar{\gamma}, \bar{\gamma})$ and $(\bar{\gamma}+20 \mathrm{~dB}, \bar{\gamma}, \bar{\gamma})$, respectively. For both the 16-QAM and 64-QAM, we observe that the performance of all CoRe schemes are worse than the conventional scheme for the case when the average SNRs are $(\bar{\gamma}, \bar{\gamma}$, $\bar{\gamma})$. Indeed, the proposed CoRe has the worst performance, since it does not assume Gary-coding in the tranmission made by the BS, which amplifies the effect of the error propagation. However, the situation is reversed for the case when the average SNRs are $(\bar{\gamma}+20 \mathrm{~dB}, \bar{\gamma}, \bar{\gamma})$. In this case, the BS-RS link is reliable enough to minimize the effect of error propagation. Nevertheless, the gain achieved by the proposed CoRe is less than the for the case of fixed relay. For the 16-QAM case, at a BER of $10^{-3}$, the proposed CoRe achieves gains of 1.6, 1 and 0.35 over the conventional, CoRe 1, and CoRe2 schemes, respectively. For the 64-QAM case, at a BER of $10^{-3}$, the proposed CoRe achieves gains of $2.5,1.1$, and 0.35 over the conventional, CoRe 1 , and CoRe2 schemes, respectively. 


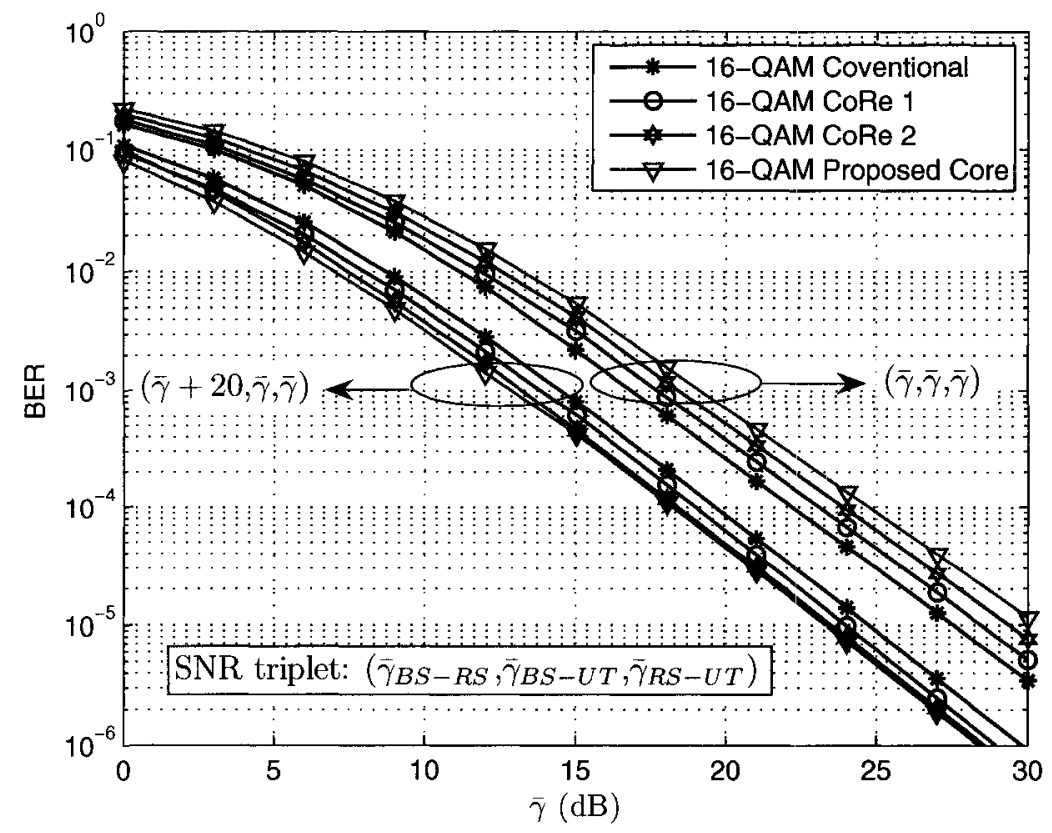

Figure 6.17: BER performance of different CoRe schemes in a nomadic relay network, using 16-QAM.

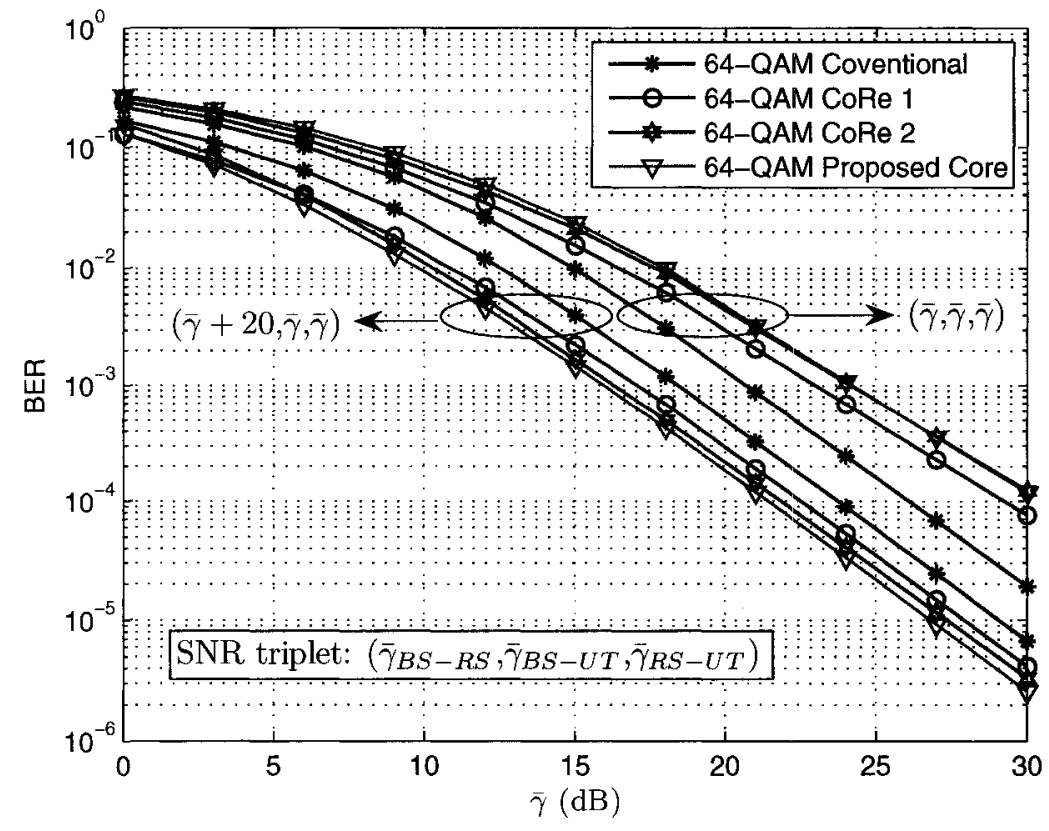

Figure 6.18: BER performance of different CoRe schemes in a nomadic relay network, using 64-QAM. 
To have a closer look at the effect of error propagation on the BER for the different CoRe schemes, we plot the BER as a function of $\bar{\gamma}_{B S-R S}$ while fixing $\bar{\gamma}_{B S-U T}$ and $\bar{\gamma}_{R S-U T}$ to be 10 and $20 \mathrm{~dB}$, respectively. This is shown in Figs. 6.19 and 6.20 for the case of 16-QAM and 64-QAM, respectively. In both figures, we observe that the proposed CoRe is the most sensitive scheme to error propagation as it suffers from the highest degradation in BER, compared to the case of fixed relay. More importantly, in order for any CoRe scheme to have better performance than the conventional scheme, $\bar{\gamma}_{B S-R S}$ must be greater than a threshold value. For the 16-QAM case, the threshold values are 25, 26.5, and 27 for CoRe 1, CoRe 2, and the proposed CoRe, respectively. For the 64-QAM case, the threshold values are 21, 22.7, and 24 for CoRe 1, CoRe 2, and the proposed CoRe, respectively. The same observations were made for different values of $\bar{\gamma}_{B S-U T}$ and $\bar{\gamma}_{R S-U T}$. However, the threshold values were different which hints that the threshold is a function of both $\bar{\gamma}_{B S-U T}$ and $\bar{\gamma}_{R S-U T}$. 


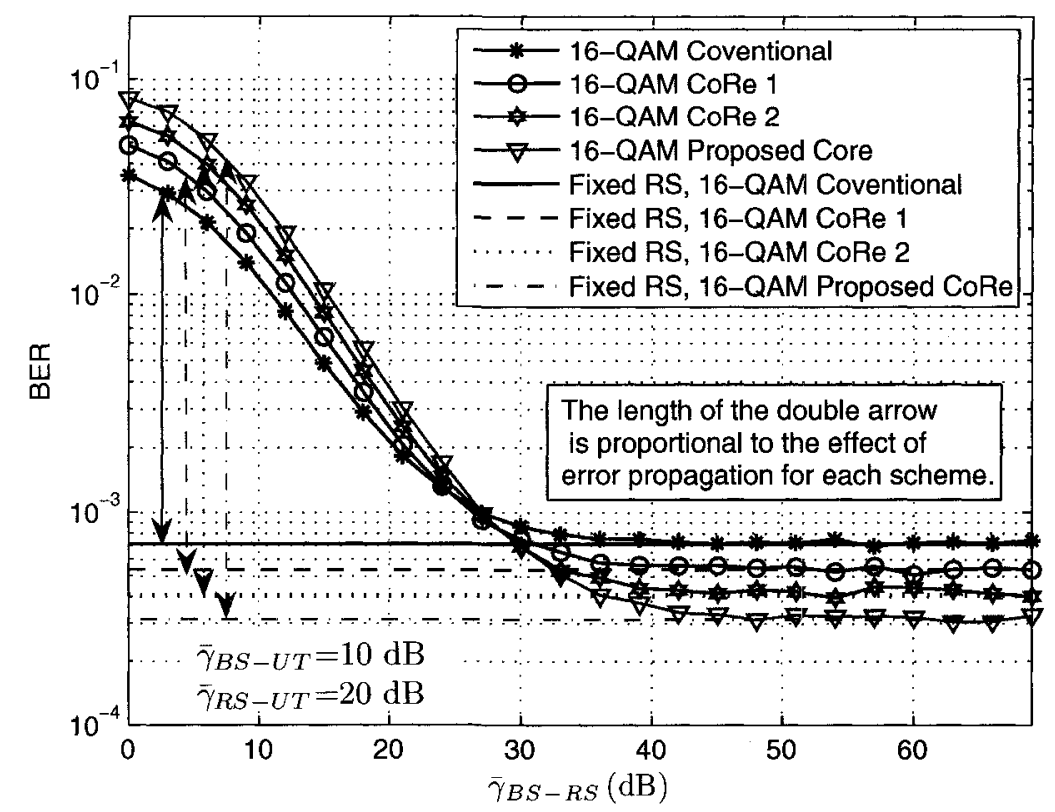

Figure 6.19: BER performance of different CoRe schemes in a nomadic relay network as a function of $\bar{\gamma}_{B S-R S}$ using 16-QAM.

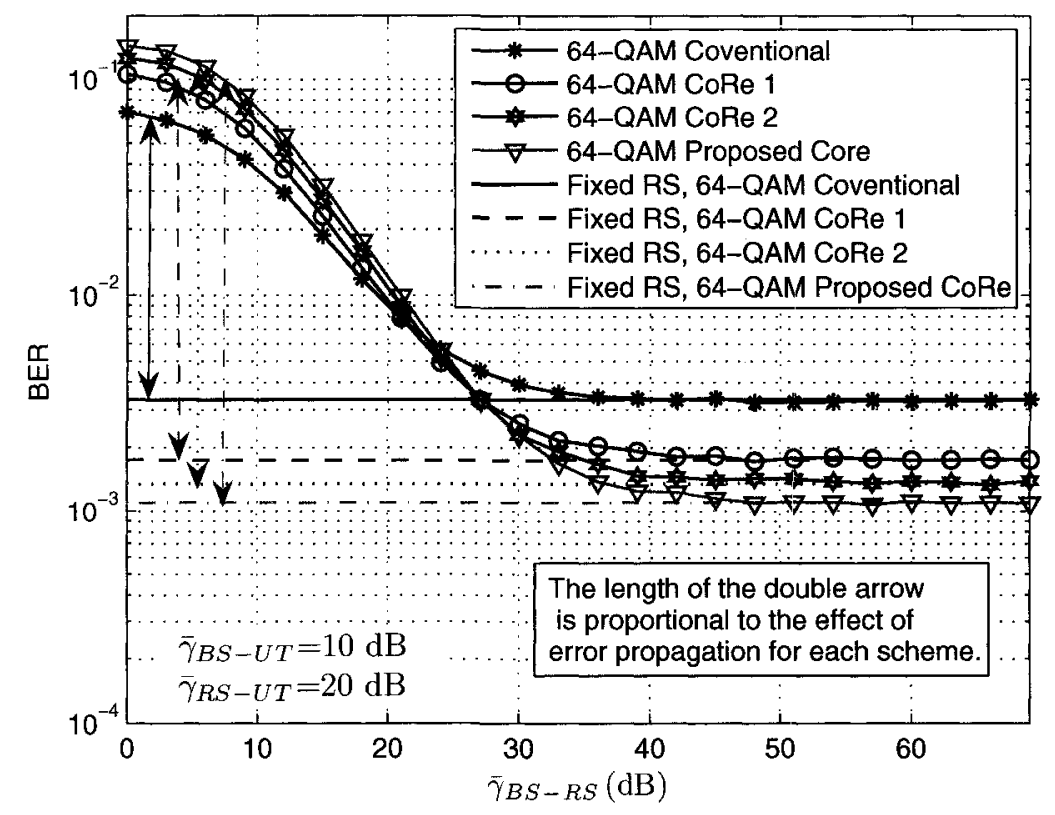

Figure 6.20: BER performance of different CoRe schemes in a nomadic relay network as a function of $\bar{\gamma}_{B S-R S}$, using 64-QAM. 


\section{Chapter 7}

\section{Conclusions and Future Work}

\subsection{Summary and Contributions}

In this thesis, we have tackled two problems in cooperative relay networks, namely, diversity combining of signals with different modulation levels and CoRe.

Problem I: Diversity combining of signals with different modulation levels

In digital cooperative relaying, signals from the source-destination and relaydestination links are combined at the destination to achieve spatial diversity. These signals do not necessarily belong to the same modulation scheme and conventional MRC does not work in such a scenario. This raises the need for a new diversity combining scheme.

\section{Problem I.1 : Fixed Relays}

The problem of diversity combining with different modulation levels is treated first by investigating different receiver structures for fixed relay networks, where the links from the BS to the RS are assumed to be error-free.

- We have introduced BSC that significantly outperforms SC in BER performance. This performance gain comes at no penalty in complexity. For both SC and BSC, we have derived closed-form BER expressions when they are used for 
combining signals with different modulation levels. The derived expression for $\mathrm{SC}$ is more general than the existing expression in literature [22, Eq. 9.210] that applies only to combining signals with the same modulation level. Moreover, we analytically quantify the significant asymptotic gain achieved by using BSC over SC.

- The optimal detector is developed as an MLD detector. To overcome the complexity of the MLD, we have investigated two other receiver structures, namely, SBMLD and SBMRC, which are bit-by-bit detectors.

- By comparing the BER performance of SC, BSC, SBMLD, and SBMRC with the optimal MLD, we have observed the followings:

- Although BSC significantly outperforms SC, its performance is still worse than that of the optimal MLD by 1.6 to $3.2 \mathrm{~dB}$, depending on the modulation levels of the signals to be combined.

- Both SBMLD and SBMRC have very close performance to the MLD scheme with a degradation that is less than $0.3 \mathrm{~dB}$. The SBMLD provides only marginal performance gain over SBMRC through the computation of the conditional probability density functions of the soft-bits.

Consequently, the SBMRC is the most attractive and practical solution.

- In addition to the simulation results of the BER performance, a very tight lower bound is derived for the BER expression of SBMRC. Since SBMRC has BER performance slightly inferior to MLD and SBMLD, the derived lower bound for SBMRC can also be used as a good approximation for the BER of both MLD and SBMLD. 
- We show that if the modulation levels are assigned by the BS based on the instantaneous CSI, and given that the assigned modulations are different, it is always beneficial to assign lower modulation levels in the links that experience better channel conditions and vice versa. Recall that such a conclusion is reversed in the conventional adaptive modulation with diversity combining. However, if assignment is done based on the average CSI, assigning lower modulation levels in the links that experience better average channel qualities does not improve the BER asymptotically.

- Besides the BER results, we show the gain in the end to end spectral efficiency using SBMRC.

\section{Problem I.2 : Nomadic Relays}

- To generalize the SBMRC for the case of nomadic relays, where error propagation is a limiting factor in the BER performance, we have implemented the LAR strategy at the relays without modifying the SBMRC structure at the UT.

- We have also introduced the modified LAR by changing the scaling coefficient in the conventional LAR.

- The simulated BER results show that SBMRC combined with LAR or modified LAR achieves full diversity. It is also shown that the modified LAR significantly outperforms the conventional LAR, without any increase in the complexity.

- We have derived tight lower bounds of the BER for both the LAR and modified LAR. 
- We show that, in the context of nomadic relays, it is always better to assign the modulation level at the BS such that it is lower or equal to the modulation level assigned to the RS, if the modulation assignments are done at the BS based on the average CSI.

\section{Problem II: CoRe in cooperative relay network}

The problem of CoRe is defined as finding good bit to symbol mapping for each transmitting node, without changing the modulation levels. Although the CoRe concept was first proposed in the context of HARQ, it can be adapted to cooperative relaying.

- Through an exhaustive numerical search, we have proposed a good CoRe scheme. Unlike most of the existing CoRe schemes, the proposed CoRe scheme does not use a Gray-coding constellation for any transmitting node.

- In the context of fixed relays, the proposed CoRe scheme shows significant gain compared to the conventional scheme and it outperforms the existing CoRe techniques.

- In the context of nomadic relays, we observe that the proposed CoRe, compared to conventional and other existing CoRe schemes, is the most sensitive scheme to error propagation as it suffers from the highest degradation in BER, compared to the case of fixed relays.

- In order for any CoRe scheme to have better performance than the conventional scheme, $\bar{\gamma}_{B S-R S}$ must be greater than a threshold value that is a function of both $\bar{\gamma}_{B S-U T}$ and $\bar{\gamma}_{R S-U T}$. Otherwise, the CoRe schemes degrade the BER 
performance as it amplifies the undesirable effect of error propagation. In nomadic relay networks, the proposed CoRe outperforms the conventional and the existing schemes if and only if $\bar{\gamma}_{B S-R S}$ is greater than this threshold.

\subsection{Future Work}

The work presented in this thesis raises some interesting topics for future research.

- In this research, we found that SBMRC is attractive in combining M-QAM modulated signals with different modulation levels, assuming all signals use the same channel coding scheme or no channel coding at all. An important extension of this work is to develop a receiver structure that is capable of combining signals with different channel coding schemes.

- To mitigate error-propagation, we have implemented LAR and we have introduced the modified LAR, and significant gain was observed. However, neither the LAR nor the modified LAR is the optimal solution for error-mitigation; this motivates the research in finding a better error-mitigation scheme.

- For cooperative relay networks, we observed that to achieve a gain from CoRe in nomadic relays, the average SNR of the source relay link must be greater than a threshold value. Through simulations, this threshold is found to be a function of the average SNR of both the relay-destination and source destination links. It is imperative to find this threshold analytically.

- The CoRe was proposed assuming the same modulation level is used for all transmitting nodes. An important extension is to consider the design of good 
bit labeling when the signals belong to different modulation levels. This point can be considered as the marriage between SBMRC and the proposed CoRe. 


\section{References}

[1] R. Pabst, B. H. Walke, D. C. Schultz, P. Herhold, H. Yanikomeroglu, S. Mukherjee, H. Viswanathan, M. Lott, W. Zirwas, M. Dohler, H. Aghvami, D. D. Falconer, and G. P. Fettweis, "Relay-based deployment concepts for wireless and mobile broadband radio," IEEE Commun. Mag., vol. 42, no. 9, pp. 80-89, September 2004.

[2] IEEE802.16, "IEEE 802.16 evaluation methodology document," IEEE 802.16, http://ieee802.org/16, Tech. Rep., August 2008.

[3] 3GPP, "Beyond 3G: LTE-advanced workshop," April 2008.

[4] J. Boyer, D. D. Falconer, and H. Yanikomeroglu, "Multihop diversity in wireless relaying channels," IEEE Trans. Commun., vol. 52, no. 10, pp. 1820-1830, Oct 2004.

[5] J. N. Laneman, D. Tse, and G. Wornell, "Cooperative diversity in wireless networks: Efficient protocols and outage behavior," IEEE Trans. On Information Theory, vol. 50, pp. 3062-3080, Dec 2004.

[6] S. M. Alamouti, "A simple transmit diversity technique for wireless communications," IEEE J. Sel. Areas Commun., vol. 16, pp. 1451-1458, October 1998.

[7] V. Tarokh, N. Seshadri, and A. Calderbank, "Space-time codes for high data rate wireless communication: Performance criteria and code construction," IEEE Trans. Inform. Theory., vol. 16, March 1998.

[8] V. Tarokh, H. Jafarkhani, and A. R.Calderbank, "Space-time block coding for wireless communications: Performance results," IEEE J. Select. Areas Commun., vol. 17, pp. 451-460, March 1998.

[9] A. Nosratinia and A. Hedayat, "Cooperative communication in wireless networks," IEEE Communication Magazine, pp. 74-80, October 2004.

[10] B. Can, H. Yanikomeroglu, F. Onat, E. Carvalho, and H. Yomo, "Efficient cooperative diversity schemes and radio resource allocation for IEEE 802.16j," IEEE Wireless Communications and Networking Conference (WCNC), April 2008. 
[11] S. Hares, H. Yanikomeroglu, and B. Hashem, "Diversity- and AMC (adaptive modulation and coding)-aware routing in TDMA peer-to-peer multihop networks," IEEE GLOBECOM, December 2003.

[12] H. Riazi, Z. Sayeed, and D. Zheng, "Signal combining scheme for wireless transmission systems having multiple modulation schemes." U.S. Patent 6580705 , June 2003.

[13] "Mobile WiMAX- PART I: A technical overview and performance evaluation," Wimax Forum, June 2006.

[14] C. Wengerter, A. G. E. von Elbwart, E. Seidel, G. Velev, and M. Schmitt, "Advanced hybrid ARQ technique employing a signal constellation rearrangement," in Proc. 56th IEEE Vehicular Technology Conference (VTC'02), Vancouver, BC, Canada, September 2002, pp. 2002 - 2006.

[15] Panasonic, "Enhanced HARQ method with signal constellation rearrangement," ftp://www.3gpp.org/tsg_ran/wg1_rl1/tsgr1_19/docs/zips/R1-010237.zip, March 2001.

[16] M. Gidlund and Y. Xu, "An improved ARQ schemes with application to multilevel modulation techniques," in Proc. IEEE ISCIT'04, Sapporo, Japan, October 2004.

[17] M. Gidlund, "Performance of combined constellation rearrangement and spacetime block coding scheme for multi-level modulation," in Proc. 18th Annual IEEE International Symposium on Personal, Indoor and Mobile Radio Communications PIMRC'07, Athens, Greece, Septmber 2007.

[18] J. Yamazaki, T. Ikeda, M. Asa, and M. M. Mollah, "Performance of symbol combining with constellation change in multihop relay," in Proc. 66th IEEE Vehicular Technology Conference-Fall (VTC'07), Baltimore, MD, USA, October 2007, pp. 1694-1697.

[19] T. Wang, R. Wang, and G. Giannakis, "Smart regenerative relays for linkadaptive cooperative communications," in Proc. 40 th Annual Conference on Information Sciences and Systems (CISS), March 2006.

[20] M. C. Jeruchim, P. Balaban, and K. S. Shanmugan, Simulation of Communication Systems: Modeling, Methodology, and Techniques, 2nd ed. New York: Kluwer/Plenum, 2000.

[21] B. Sklar, Digital Communications: Fundamentals and Applications, 2nd ed. Upper Saddle River, NJ: Prentice-Hall, 2001. 
[22] M. K. Simon and M. Alouini, Digital Communication over Fading Channels: A Unified Approach to Performance Analysis. Wiley \& Sons, Inc., 2000.

[23] J. G. Proakis, Digital Coomunications. McGraw-Hill, 2000.

[24] F. A. Onat, A. Adinoyi, Y. Fan, H. Yanikomeroglu, J. S. Thompson, and I. D. Marsland, "Threshold selection for SNR-based selective digital relaying in cooperative wireless networks," Accepted for publication in IEEE Transactions on Wireless Communications, Dec 2007.

[25] R. D. Wesel, X. Liu, J. M. Cioffi, and C. Komninakis, "Constellation labeling for linear encoders," IEEE Trans. Inf. Theory, vol. 47, no. 6, pp. 2417-2431, Sep 2001.

[26] J. Castura, "Rateless coding over wireless channels: Theory, design and applications," Ph.D. dissertation, University of Ottawa, Faculty of Engineering, School of Information Technology and Engineering, 2008.

[27] S. L. Goff, A. Glavieux, and C. Berrou, "Turbo-codes and high spectral efficiency modulation," in Proc. ICC'94, New Orleans, LA, USA, May 1994.

[28] V. Aue and R. Nuessgen, "Method for generating soft-bit information from gray coded signals." U.S. Patent 20040096007, May 2004.

[29] K. S. Kim, K. Hyun, C. W. Yu, Y. O. Park, D. Yoon, and S. K. Park, "General log-likelihood ratio expression and its implementation algorithm for Gray-Coded QAM signals," ETRI Journal, vol. 28, no. 3, pp. 291-300, June 2006.

[30] A. Bin-Sediq and H. Yanikomeroglu, "Diversity combining of signals with different modulation levels in cooperative relay networks," in IEEE VTC2008-Fall, Calgary, Alberta, Canada, September 2008.

[31] - "Diversity combining of signals with different modulation levels in cooperative relay networks," in Wireless World Research Forum Meeting 20 (WWRF20), Ottawa, Canada, April 2008.

[32] A. Leon-Garcia, Probability and Random Processes for Electrical Engineering, 2nd ed. Addison-Wesley, 1994.

[33] Herhold, E. Zimmermann, and G. Fettweis, "A simple cooperative extension to wireless relaying," in Proc. International Zurich Seminar on Communications, 2004, p. 3639 . 
[34] A. Adinoyi and H. Yanikomeroglu, "Multi-antenna aspects of wireless fixed relays," in Proc. IEEE Wireless Communications and Networking Conference (WCNC), April 2006.

[35] W. P. Siriwongpairat, T. Himsoon, W. Su, and K. J. R. Liu, "Optimum threshold-selection relaying for decode-and-forward cooperation protocol," in Proc. IEEE Wireless Communications and Networking Conference (WCNC), April 2006.

[36] J. N. Laneman and G. W. Wornell, "Distributed space-time-coded protocols for exploiting cooperative diversity in wireless networks," IEEE Trans. on Information Theory, vol. 49, pp. 2415-2425, Oct 2003.

[37] T. Wang, A. Cano, and G. Giannakis, "Link-adaptive cooperative communications without channel state information," in Proc. Military Communications Conference (MILCOM), October 2006.

[38] F. A. Onat, Y. Fan, H. Yanikomeroglu, and J. S. Thompson, "Asymptotic BER analysis of threshold digital relaying in cooperative wireless systems," Accepted for publication in IEEE Transactions on Wireless Communications, Dec 2007.

[39] D. Chen and J. N. Laneman, "Modulation and demodulation for cooperative diversity in wireless systems," IEEE Transactions on Wireless Communications, vol. 5, pp. 1785-1794, July 2006.

[40] T. Wang, A. Cano, G. B. Giannakis, and J. N. Laneman, "High-performance cooperative demodulation with decode-and-forward relays," IEEE Transactions on Communications, vol. 55, pp. 1427-1438, Dec 2007.

[41] M. Chiani and D. Dardari, "Improved exponential bounds and approximation for the Q-function with application to average error probability computation," in Global Telecommunications Conference, November 2002.

[42] M. Abramowitz and I. A. Stegun, Handbook of Mathematical Functions. New York: Dover Publications, 1965. 


\section{Appendix A}

\section{Confidence Interval Analysis}

To obtain the confidence interval, we use the normal approximation explained in [20]. Let us call the BER estimator $\hat{p}$ obtained by sending $N$ bits using Monte Carlo simulation. The confidence interval, with a confidence level of $\alpha \%$, is:

$$
\begin{aligned}
& \hat{p} y_{-} \leq \hat{p} \leq \hat{p} y_{+} \\
& y_{ \pm}=\left(1+\frac{z_{\alpha}^{2}}{2 N \hat{p}}\left(1 \pm \sqrt{4 N \hat{p} / z_{\alpha}^{2}+1}\right)\right)
\end{aligned}
$$

where $z_{90}=0.6449, z_{95}=1.96$ and $z_{99}=0.5758$. We define the normalized confidence interval as $\left(y_{-}, y_{+}\right)$. The normalized confidence interval is more informative than the confidence interval itself [20].

From the previous equation, we notice that in order to improve the accuracy of the estimator $\hat{p}, N \hat{p}$, which represents the number of errors observed, should be as high as possible. Consequently, the Monte Carlo simulation algorithm was built as follows. It sets $N=10^{6}$, and it calculates $N \hat{p}$. If $N \hat{p}$ is greater than 300 , the simulation terminates and the value of $\hat{p}$ is recorded as a good estimate of the BER . If not, the simulation repeats and it accumulates the number of errors $N \hat{p}$ until it is equal or greater than 300 . The rational behind building the simulator in this fashion is to avoid unnecessary long simulation time for large $\hat{p}$ while sustaining reasonable accuracy for low $\hat{p}$. 
For this simulator, using (A.1), the normalized confidence interval is $\left(y_{-}, y_{+}\right)$ where

$$
y_{ \pm}=\left\{\begin{array}{rl}
1+\frac{z_{\alpha}^{2}}{2 \times 10^{6} \hat{p}}\left(1 \pm \sqrt{4 \times 10^{6} \hat{p} / z_{\alpha}^{2}+1}\right) & \hat{p} \geq 3 \times 10^{-4} \\
1+\frac{z_{\alpha}^{2}}{600}\left(1 \pm \sqrt{1200 / z_{\alpha}^{2}+1}\right) & \hat{p}<3 \times 10^{-4}
\end{array} .\right.
$$

The previous equation is plotted for different confidence levels in the following figure. From the figure, with a confidence level of $95 \%$, the implemented simulator estimates the actual BER with an inaccuracy of utmost $\pm 6 \%$ at BER of $10^{-3}$ and an inaccuracy of utmost $\pm 12 \%$ at a BER of $10^{-6}$, which are acceptable inaccuracies for all practical purposes [20].

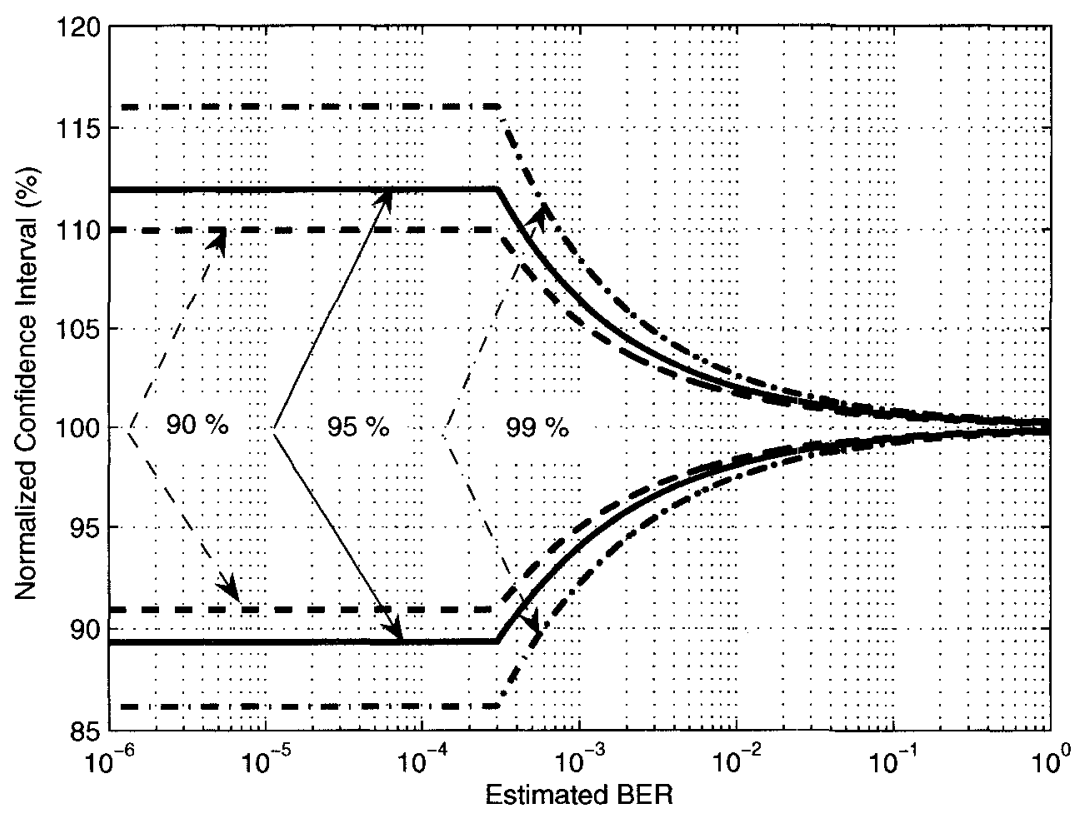

Figure A.1: The normalized confidence interval of the implemented simulator for different confidence levels and at different BER values. 
Appendix B

\section{Different Gray Coded 16-QAM Constellations}

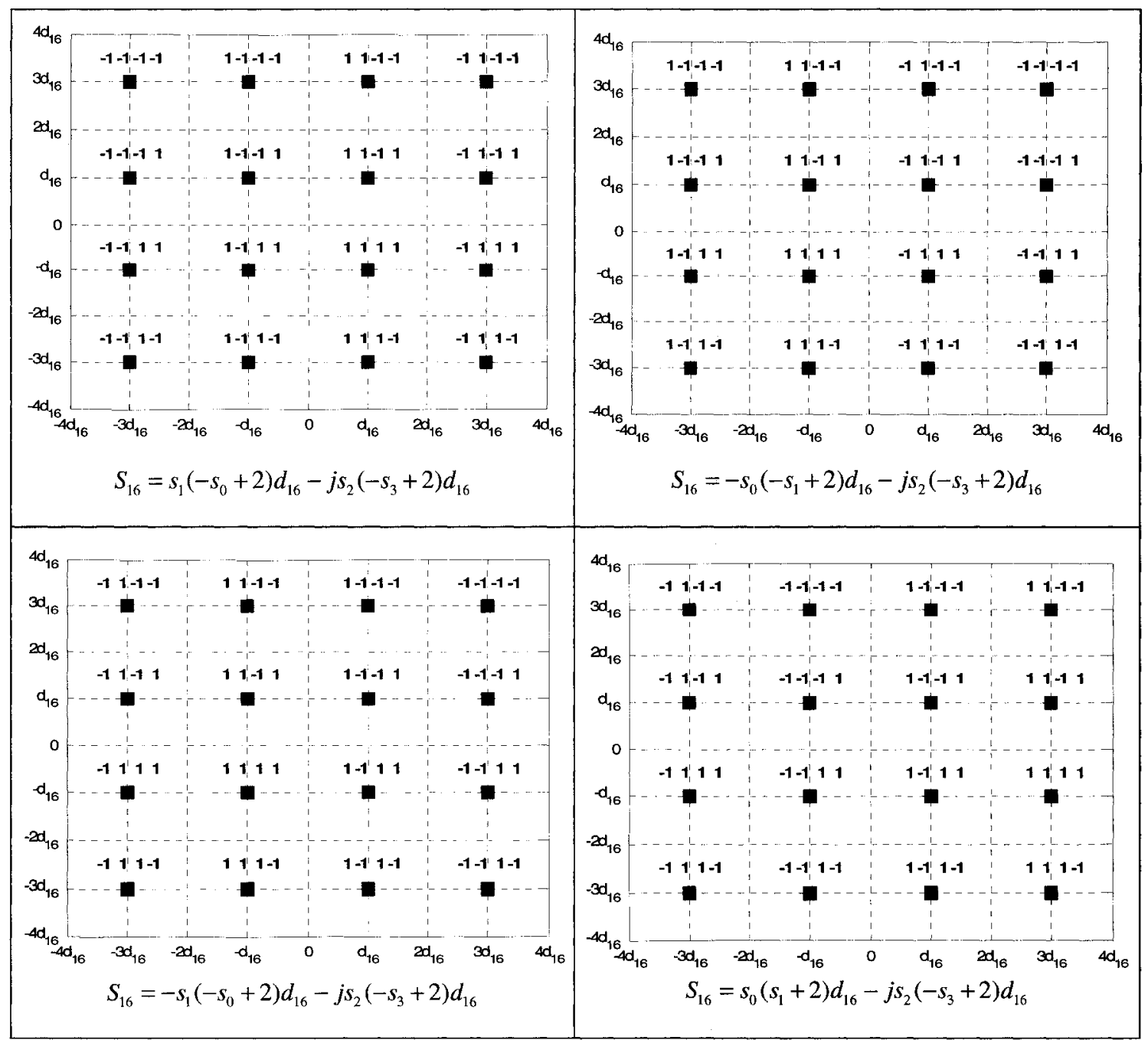




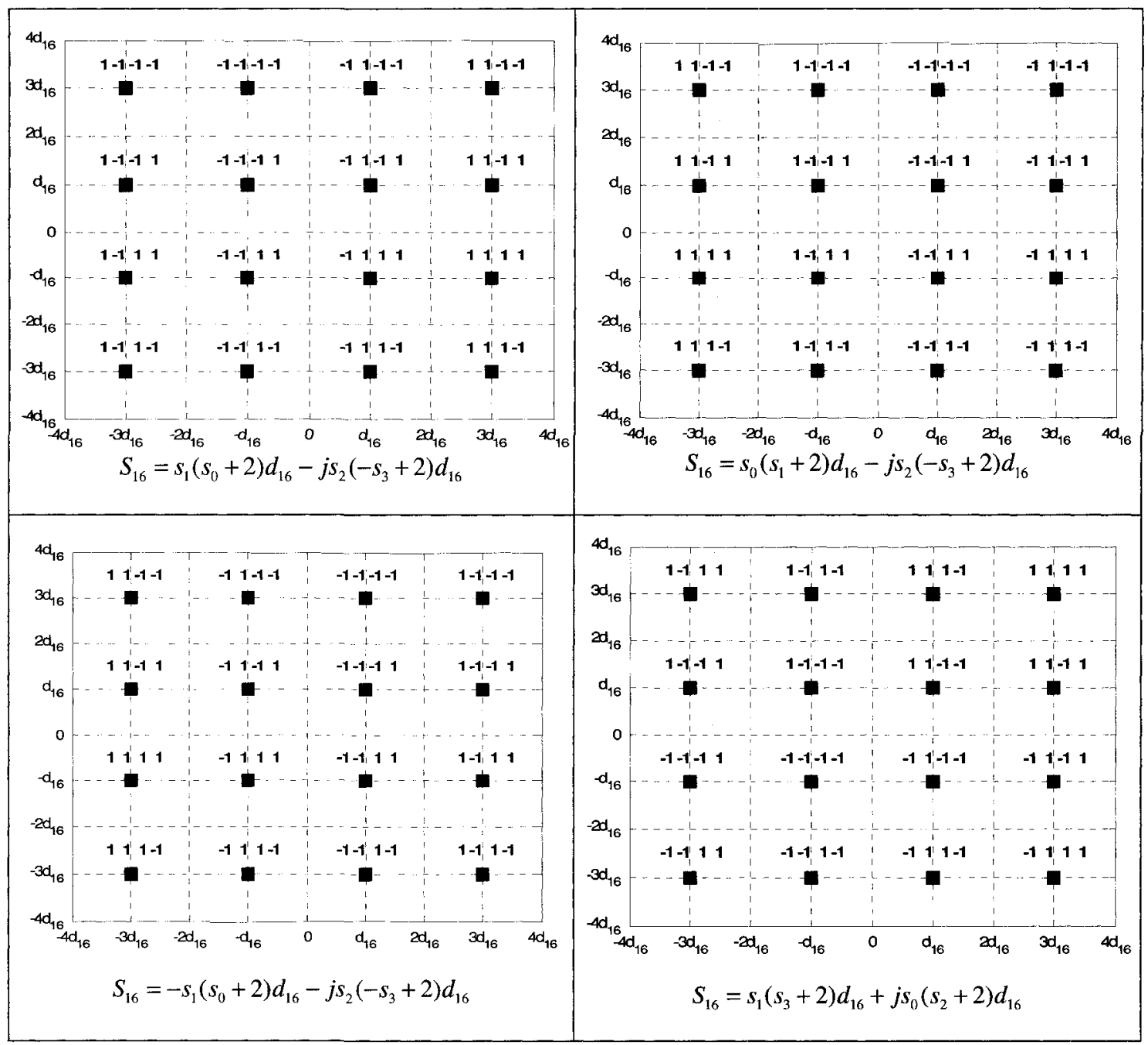

Figure B.1: Different Gray coded 16-QAM constellations with their mathematical models. 


\section{Appendix C}

\section{Derivation of $I_{0}$ and $I_{1}$}

\section{C.1 Finding $I_{0}$}

By substituting (5.4) and (5.13) in (5.10), we get

$$
I_{0}=I_{0,0}+I_{0,1}, \text { where }
$$

$$
\begin{aligned}
& I_{0,0}=\int_{0}^{\rho \bar{\gamma}_{R S-U T}} \int_{0}^{\infty}\left(1-c_{M_{0}} Q\left(\sqrt{2 d_{M_{0}}^{2} \gamma_{B S-R S}}\right)\right) \tau Q\left(\sqrt{2 \gamma_{\text {out }}}\right) \times \\
& \frac{1}{d_{M_{0}}^{2} \bar{\gamma}_{B S-U T}-d_{M_{1}}^{2}\left(\gamma_{B S}-R S / \rho\right)}\left(e^{-\frac{\gamma_{\text {out }}}{d_{M_{0}}^{2} \bar{\gamma}_{B S}-U T}}-e^{-\frac{\rho \gamma_{\text {out }}}{d_{M_{1}}^{2} \gamma_{B S}-R S}}\right) \frac{1}{\bar{\gamma}_{B S-R S}} e^{-\frac{\gamma_{B S-R S}}{\bar{\gamma}_{B S}-R S}} d \gamma_{\text {out }} d \gamma_{B S-R S} \\
& I_{0,1}=\int_{\rho \bar{\gamma}_{R S-U T}}^{\infty} \int_{0}^{\infty}\left(1-c_{M_{0}} Q\left(\sqrt{2 d_{M_{0}}^{2} \gamma_{B S-R S}}\right)\right) \tau Q\left(\sqrt{2 \gamma_{o u t}}\right) \times \\
& \frac{1}{d_{M_{0}}^{2} \bar{\gamma}_{B S-U T}-d_{M_{1}}^{2} \bar{\gamma}_{R S-U T}}\left(e^{-\frac{\gamma_{\text {out }}}{d_{M_{0}}^{2} \bar{\gamma}_{B S-U T}}}-e^{-\frac{\gamma_{d_{1}}^{2} \bar{\gamma}_{R S}}{\bar{\gamma}_{R S T}}}\right) \frac{1}{\bar{\gamma}_{B S-R S}} e^{-\frac{\gamma_{B S-R S}}{\bar{\gamma}_{B S}-R S}} d \gamma_{\text {out }} d \gamma_{B S-R S} .
\end{aligned}
$$

The integral $I_{0,1}$ can be evaluated as follows

$$
\begin{aligned}
& I_{0,1}=\int_{\rho \bar{\gamma}_{R S-U T}}^{\infty}\left(1-c_{M_{0}} Q\left(\sqrt{2 d_{M_{0}}^{2} \gamma_{B S-R S}}\right)\right) \frac{1}{\bar{\gamma}_{B S-R S}} e^{-\frac{\gamma_{B S-R S}}{\bar{\gamma}_{B S-R S}} d \gamma_{B S-R S} \times} \\
& \int_{0}^{\infty} \tau Q\left(\sqrt{2 \gamma_{o u t}}\right) \frac{1}{d_{M_{0}}^{2} \bar{\gamma}_{B S-U T}-d_{M_{1}}^{2} \bar{\gamma}_{R S-U T}}\left(e^{-\frac{\gamma_{\text {out }}}{d_{M_{0}}^{2} \bar{\gamma}_{B S-U T}}}-e^{-\frac{\gamma_{\text {out }}}{d_{M_{1}}^{2} \bar{\gamma}_{R S-U T}}}\right) d \gamma_{o u t} \\
& =e^{-\frac{\rho \bar{\gamma}_{R S-U T}}{\bar{\gamma}_{B S-U T}}} Q\left(\sqrt{2 d_{M_{0}}^{2} \frac{\rho \bar{\gamma}_{R S-U T}}{\bar{\gamma}_{B S-R S}}}\right)-\sqrt{\frac{d_{M_{0}}^{2} \bar{\gamma}_{B S-R S}}{1+d_{M_{0}}^{2} \bar{\gamma}_{B S-R S}}} Q\left(\sqrt{\frac{\rho \bar{\gamma}_{R S-U T}\left(1+d_{M_{0}}^{2} \bar{\gamma}_{B S-R S}\right)}{\bar{\gamma}_{B S-R S}}}\right) \times \\
& \left(d_{M_{0}}^{2} \bar{\gamma}_{B S-U T}\left(1-\sqrt{\frac{d_{M_{0}}^{2} \bar{\gamma}_{B S-U T}}{1+d_{M_{0}}^{2} \bar{\gamma}_{B S-U T}}}\right)-d_{M_{1}}^{2} \bar{\gamma}_{R S-U T}\left(1-\sqrt{\frac{d_{M_{1}}^{2} \bar{\gamma}_{R S-U T}}{1+d_{M_{1}}^{2} \bar{\gamma}_{R S-U T}}}\right)\right) \times \\
& \bar{d}_{M_{0}}^{2} \overline{\bar{\gamma}}_{B S-U T}-d_{M_{1}}^{2} \bar{\gamma}_{R S-U T}
\end{aligned}
$$


where the first integral is evaluated using (3.7) and the second integral represents the average BER of fixed RS and it is given by (4.31). The integral $I_{0,0}$ is difficult to evaluate in closed-form. To rectify this problem, we approximate the $Q$ functions involved in the integration by using the approximation given by [41, Eq. 14] as follows

$$
\begin{aligned}
& Q\left(\sqrt{2 \gamma_{o u t}}\right) \approx \sum_{m=0}^{1} a_{m} e^{-b_{m} \gamma_{o u t}} \\
& Q\left(\sqrt{2 d_{M_{0}}^{2} \gamma_{B S-R S}}\right) \approx \sum_{n=0}^{1} a_{n} e^{-b_{n} d_{M_{0}}^{2} \gamma_{B S-R S}}
\end{aligned}
$$

where $a_{0}=\frac{1}{12}, a_{1}=\frac{1}{4}, b_{0}=1$ and $b_{1}=\frac{4}{3}$.

By substituting (C.5) in (C.2), and after manipulation and utilization of the linearity of the summation and integration, we get

$$
\begin{aligned}
& I_{0,0}=\sum_{n=0}^{1} \sum_{m=0}^{1} \int_{0}^{\rho \bar{\gamma}_{R S-U T}} \int_{0}^{\infty}\left(\frac{1}{2}-c_{M_{0}} a_{n} e^{-b_{n} d_{M_{0}}^{2} \gamma_{B S-R S}}\right) \tau a_{m} e^{-b_{m} \gamma_{\text {out }}} \times \\
& \frac{1}{d_{M_{0}}^{2} \bar{\gamma}_{B S-U T}-d_{M_{1}}^{2}\left(\gamma_{B S-R S} / \rho\right)}\left(e^{-\frac{\gamma_{\text {out }}}{d_{M_{0}}^{2} \bar{\gamma}_{B S-U T}}}-e^{-\frac{\rho \gamma_{0 u t}}{d_{M_{1}}^{2} \gamma_{B S}-R S}}\right) \frac{1}{\bar{\gamma}_{B S-R S}} e^{-\frac{\gamma_{B S-R S}}{\bar{\gamma}_{B S}-R S}} d \gamma_{\text {out }} d \gamma_{B S-R S}
\end{aligned}
$$

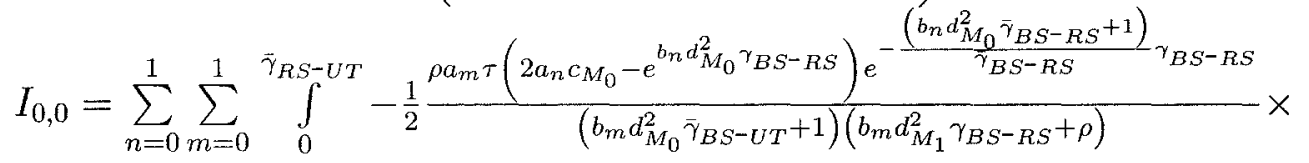

$$
\begin{aligned}
& \frac{1}{\bar{\gamma}_{B S-R S}} e^{-\frac{\gamma_{B S-R S}}{\bar{\gamma}_{B S}-R S}} d \gamma_{B S-R S}
\end{aligned}
$$

Using the exponential integral function Ei defined as [42]

$$
\operatorname{Ei}(x)=\int \frac{e^{-x}}{-x} d x
$$

the integration given by (C.6) can be written as follows

$$
\begin{aligned}
& I_{0,0}=\sum_{n=0}^{1} \sum_{m=0}^{1} P_{m, n} \text { where } \\
& P_{m, n}=\frac{\rho a_{m} \tau}{2 \bar{\Gamma}_{2}\left(\bar{\Gamma}_{0}+1\right)} e^{\frac{\rho}{\Gamma_{2}}}\left(\begin{array}{l}
\operatorname{Ei}\left(\frac{\rho\left(\bar{\Gamma}_{1}+1\right)}{\Gamma_{2}}\right)-\operatorname{Ei}\left(\frac{\rho}{\Gamma_{2}}\right)+ \\
2 a_{n} c_{M_{0}} e^{\frac{\rho b_{n} d_{M_{0}}^{2} d_{M_{1}}^{2}}{b_{2}}}\left(\operatorname{Ei}\left(\frac{\rho\left(\bar{\Gamma}_{3}+1\right)}{\Gamma_{2}}\right)-\operatorname{Ei}\left(\frac{\rho\left(\bar{\Gamma}_{3}+1\right)\left(\bar{\Gamma}_{1}+1\right)}{\Gamma_{2}}\right)\right)
\end{array}\right), \\
& \bar{\Gamma}_{0}=b_{m} d_{M_{0}}^{2} \bar{\gamma}_{B S-U T}, \bar{\Gamma}_{1}=b_{m} d_{M_{1}}^{2} \bar{\gamma}_{R S-U T}, \bar{\Gamma}_{2}=b_{m} d_{M_{1}}^{2} \bar{\gamma}_{B S-R S} \text { and } \bar{\Gamma}_{3}=b_{n} d_{M_{0}}^{2} \bar{\gamma}_{B S-R S .} .
\end{aligned}
$$




\section{C.2 Finding $I_{1}$}

Using (5.12) and (3.7), it is straight forward to show that

$$
I_{1}=\frac{1}{4}\left(1-\sqrt{\frac{d_{M_{0}}^{2} \bar{\gamma}_{B S-R S}}{1+d_{M_{0}}^{2} \bar{\gamma}_{B S-R S}}}\right)\left(1-\sqrt{\frac{d_{M_{0}}^{2} \bar{\gamma}_{R S-U T}}{1+d_{M_{0}}^{2} \bar{\gamma}_{R S-U T}}}\right)
$$




\section{Appendix D}

\section{Proof that it is Always Better to Assign $M_{0} \leq M_{1}$}

In this appendix, we show that in the context of nomadic relays, it is always better to assign the modulation level at the BS such that it is lower or equal to the modulation level assigned to the RS, if the modulation assignments are done at the BS based on the average channel conditions.

We define two strategies, 1 and 2. In both strategies, the BS and RS use the modulation levels of $M$ and $N$, where $M<N$. Consequently, both strategies achieve the same end-to-end spectral efficiency of $\delta=\left(\left(\log _{2} M\right)^{-1}+\left(\log _{2} N\right)^{-1}\right)^{-1}$, which implies that $\delta$ is a neutral factor in the comparison between the two strategies. In strategy 1 , the BS transmits using $M$-QAM, while the RS transmits using $N$-QAM. In strategy 2, the BS transmits using $N$-QAM, while the RS transmits using $M$ QAM. The average BERs using SBMRC at the UT are denoted by $B E R_{1}$ and $B E R_{2}$ for strategy 1 and 2, respectively. Our objective is to prove that

$$
B E R_{1}<B E R_{2}
$$

regardless of the average SNRs of the different links, which means that strategy 1 is always better than strategy 2 .

Using the results in [24], the average BER for both strategies can be written as:

$$
\begin{aligned}
& B E R_{1}=\left(1-B E R_{1}^{B S-R S}\right) B E R_{1}^{c o o p}+B E R_{1}^{B S-R S} B E R_{1}^{p r o p} \\
& B E R_{2}=\left(1-B E R_{2}^{B S-R S}\right) B E R_{2}^{c o o p}+B E R_{2}^{B S-R S} B E R_{2}^{p r o p}
\end{aligned}
$$


where

$B E R_{i}^{B S-R S}$ : The average BER in the BS - RS link

$B E R_{i}^{c o o p}$ : The average BER at UT after combining using SBMRC, given that no error happend at RS

$B E R_{i}^{\text {prop }}$ : The average BER at UT after combining using SBMRC, given that RS has detection error

for the strategy $i$.

It is easy to see that

$$
B E R_{1}^{B S-R S}<B E R_{2}^{B S-R S}
$$

for any $\bar{\gamma}_{B S-R S}$, since the modulation level used in the BS in strategy 1 is lower, thus it has less vulnerability to noise.

Using the asymptotically tight lower bound derived in (4.32), we can write:

$$
B E R_{1}^{\text {coop }} \simeq B E R_{2}^{\text {coop }}
$$

where $\simeq$ means asymptotically equal.

Since there is no closed form expression of $B E R_{i}^{\text {prop }}$ for strategy $i$, we resort to the following argument. In strategy 1, when there is a detection error at RS, this error propagates to the UT with the higher level modulation, and the correct symbols transmitted from the BS to the UT with the lower level modulation. Since SBMRC weights the higher level modulated symbols with less weight, the effect of the error propagated from the RS will be reduced, as the SBMRC will emphasize the symbols from the BS more.

In strategy 2 , when there is a detection error at RS, this error propagates to the UT with the lower level modulation, and the correct symbols transmitted from the 
BS to the UT with the higher level modulation. Since SBMRC weights the lower level modulated symbols with more weight, the effect of the error propagated from the RS will be amplified, as the SBMRC will emphasize the symbols from the RS more.

Consequently, we can write the following

$$
B E R_{1}^{\text {prop }}<B E R_{2}^{\text {prop }}
$$

By subsituiting (D.2), (D.3) and (D.4) in (D.1) we can conclude that

$$
B E R_{1}<B E R_{2}
$$

Although this conclusion is strictly valid asymptotically, the simulation results show that it is valid for any SNR. 